\title{
Inverse Problems in Local Helioseismology
}

\author{
Dissertation \\ zur Erlangung des mathematisch-naturwissenschaftlichen Doktorgrades \\ "Doctor rerum naturalium" \\ der Georg-August-Universität Göttingen \\ im Promotionsprogramm PROPHYS \\ der Georg-August University School of Science (GAUSS)
}

vorgelegt von

Majid Pourabdian

aus Rasht, Iran

Göttingen, 2020 
Betreuungsausschuss

\section{Prof. Dr. Laurent Gizon}

Max-Planck-Institut für Sonnensystemforschung und

Institut für Astrophysik, Georg-August-Universität Göttingen, Deutschland

\section{Prof. Dr. Thorsten Hohage}

Institut für Numerische und Angewandte Mathematik, Georg-August-Universität Göttingen und

Max-Planck-Institut für Sonnensystemforschung, Göttingen, Deutschland

\section{Dr. Damien Fournier}

Max-Planck-Institut für Sonnensystemforschung, Göttingen, Deutschland

Mitglieder der Prüfungskommision

Referent: Prof. Dr. Laurent Gizon

Max-Planck-Institut für Sonnensystemforschung und

Institut für Astrophysik, Georg-August-Universität Göttingen, Deutschland

Korreferent: Prof. Dr. Thorsten Hohage

Institut für Numerische und Angewandte Mathematik, Georg-August-Universität Göttingen und

Max-Planck-Institut für Sonnensystemforschung, Göttingen, Deutschland

Weitere Mitglieder der Prüfungskommission:

Prof. Dr. Stefan Dreizler

Institut für Astrophysik, Georg-August-Universität Göttingen, Deutschland

Prof. Dr. Andreas Tilgner

Institut für Geophysik, Georg-August-Universität Göttingen, Deutschland

Prof. Dr. Wolfram Kollatschny

Institut für Astrophysik, Georg-August-Universität Göttingen, Deutschland

Prof. Dr. Ramin Yahyapour

Gesellschaft für wissenschaftliche Datenverarbeitung mbH Göttingen und Institut für Informatik, Georg-August-Universität Göttingen, Deutschland

Tag der mündlichen Prüfung: 17.02.2020 


\section{Bibliografische Information der Deutschen Nationalbibliothek}

Die Deutsche Nationalbibliothek verzeichnet diese Publikation in der Deutschen Nationalbibliografie; detaillierte bibliografische Daten sind im Internet über http: //dnb . d-nb . de abrufbar.

(C) Majid Pourabdian

(c) Th
Cry
Creative Commons Attribution 4.0 License

Printed in Germany 
Cover figure: meridional flow inferred using inversions of MDI and GONG helioseismic travel times for solar cycles 23 and 24. This figure is adapted from Fig. 2A of Gizon et al. (2020). 


\section{Contents}

\begin{tabular}{ll}
\hline Summary & 7
\end{tabular}

\begin{tabular}{lll}
\hline & Introduction & 9
\end{tabular}

1.1 Solar meridional circulation $\ldots \ldots \ldots$. . . . . . . . . . . . 9

1.2 Solar oscillations and helioseismology . . . . . . . . . . . . . . . . . . . . . . . . . 12

1.3 The forward and inverse problems . . . . . . . . . . . . . . . . 17

1.4 Contents of this thesis $\ldots \ldots \ldots \ldots$. . . . . . . . . . . 20

2 Comparison of travel-time and amplitude measurements for deep-focusing $\begin{array}{ll}\text { time-distance helioseismology } & 21\end{array}$

2.1 Introduction . . . . . . . . . . . . . . . . . . . . 21

2.2 Travel-time and amplitude measurements f............ 23

2.2 .1 Definitions . . . . . . . . . . . . . . . 23

2.2 .2 Deep-focusing averages . . . . . . . . . . . . . . 24

2.2 .3 Noise model . . . . . . . . . . . . . . . . . . . . 24

2.3 Travel-time and amplitude sensitivity kernels for sound-speed perturba-

tions to a uniform background medium . . . . . . . . . . . . 26

2.3 .1 Wave equation and reference Green's function . . . . . . . . 26

2.3 .2 Perturbation to the cross-covariance function . . . . . . . . . . 26

2.3 .3 Travel-time and amplitude sensitivity kernels . . . . . . . . . 27

2.4 Example calculations . . . . . . . . . . . . . . . . . 28

2.4 .1 Choice of numerical values and parameters . . . . . . . . 28

2.4 .2 Point-to-point sensitivity kernels . . . . . . . . . . . . . . . . . . . . . . . . 28

2.4 .3 Deep-focusing sensitivity kernels . . . . . . . . . . . . . . . . 28

2.4 .4 Kernel widths as functions of target depth . . . . . . . . . . . . . 30

2.4 .5 Noise covariance . . . . . . . . . . . . . . . . . 33

$2.4 .6 \quad$ Localized sound-speed anomaly at $z_{0}=0.7 \mathrm{R}_{\odot} \ldots \ldots$. . . . . . 33

2.4 .7 Sound-speed anomaly in a shell at radius $r_{0}=0.7 \mathrm{R}_{\odot} \ldots . . .35$

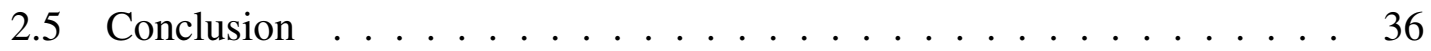

\begin{tabular}{|lll}
3 & Single-cell meridional flow in the solar convection zone & 37
\end{tabular}

3.1 Report . . . . . . . . . . . . . . . . . . . 37

3.2 Supplementary materials: Materials and methods . . . . . . . . . . . . . . 45

3.2.1 Helioseismic travel times . . . . . . . . . . . . . . . . . . . . . . . 45

3.2 .2 Discretization of the meridional flow . . . . . . . . . . . 47

$3.2 .3 \quad$ Forward problem . . . . . . . . . . . . . . . . . . 47 
3.2 .4 Inverse problem . . . . . . . . . . . . . . . . . . . . . . . . 48

3.2 .5 Tests with synthetics . . . . . . . . . . . . . . . . . . 49

3.2 .6 Inversions of observed travel times . . . . . . . . . . . . 50

$3.2 .7 \quad$ Flux-transport dynamo model $\ldots \ldots \ldots$. . . . . . . . 50

3.3 Supplementary materials: Supplementary figures . . . . . . . . . . . . 52

4 Helioseismic inversions for the solar meridional flow using synthetic data 65

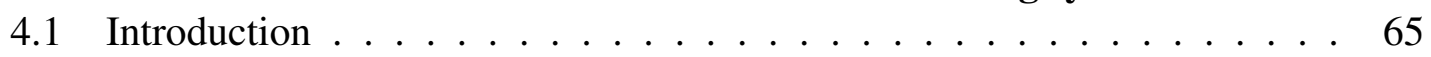

$4.2 \quad$ Forward problem $\ldots \ldots \ldots \ldots$. . . . . . . . . . . . 66

4.2.1 Modeling travel-time measurements using Born sensitivity kernels 67

4.2 .2 Discretization of the model . . . . . . . . . . . . . 68

4.2 .3 Noise . . . . . . . . . . . . . . . . . . . . . . . . 69

4.3 Inversion setup $\ldots \ldots \ldots \ldots$

4.3 .1 Regularization term . . . . . . . . . . . . . . . . 69

4.3 .2 Inversion with constraints . . . . . . . . . . . . 71

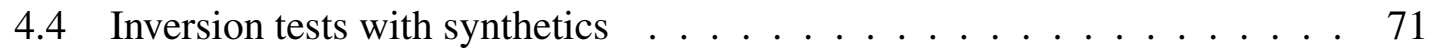

4.4 .1 Synthetic travel times . . . . . . . . . . . . . . . . . . . . . . . . 71

4.4 .2 Tests for mass conservation . . . . . . . . . . . . 72

4.4 .3 Tests for regularization term . . . . . . . . . . . . 72

4.4.4 Tests for the boundary constraint . . . . . . . . . . . . . . . 75

4.4 .5 Tests for the effect of large separation distances . . . . . . . . . 75

4.5 Conclusion . . . . . . . . . . . . . . . . 78

$\begin{array}{lll}5 & \text { Discussion } & 81\end{array}$

5.1 Helioseismic observables and forward modeling . . . . . . . . . . . . 81

5.2 Comparison of inversion strategies . . . . . . . . . . . . . . . . . . . . . . . . 84

5.3 Conclusions $\ldots \ldots \ldots \ldots \ldots \ldots$

\begin{tabular}{lr}
\hline Bibliography & 91
\end{tabular}

\begin{tabular}{ll}
\hline Scientific contributions & 101
\end{tabular}

\begin{tabular}{ll}
\hline Acknowledgements & 103
\end{tabular}

\begin{tabular}{ll}
\hline Curriculum vitae & 105
\end{tabular} 


\section{Summary}

Helioseismology is the main tool to infer the physical properties in the solar interior. In time-distance helioseismology, measurements of wave travel times are extracted from the cross-correlation of the oscillation signal (e.g. the Doppler velocity) between pairs of points on the solar surface. These measurements must then be inverted (the inverse problem) to infer the solar subsurface properties. Helioseismic inferences are based on a relationship between the perturbations in solar properties with respect to a reference solar model and the corresponding changes in the helioseismic measurements (the forward problem). Measurements of wave travel times are very noisy and suffer from systematic errors. These have led to conflicting results, in particular in the deeper layers of the Sun and many open questions about the solar internal structure. A particularly challenging problem is the inference of the solar meridional flow, which is a crucial ingredient in models of the solar dynamo. There is no consensus about the radial profile of the solar meridional flow. This dissertation mainly focuses on a better understanding of the solar meridional flow deep inside the convection zone by performing helioseismic inversions of wave travel times. In doing so, improved methods of inversion are developed.

In a first study, we consider acoustic waves propagating in a homogeneous medium to investigate the deep-focusing time-distance technique in terms of signal and noise. The aim of the deep-focusing time-distance helioseismology is to construct seismic measurements that inform us about the physical conditions at a well-defined target point in the solar interior. In this technique, pairs of points on the solar surface are chosen in a way that their acoustic ray paths intersect at the target point. We compare two measurement quantities extracted from the deep-focusing cross-covariance functions: travel times and amplitudes. Using the first Born approximation which is a single-scattering approximation, we find the deep-focusing travel-time measurements have zero sensitivity at the target location and maximum sensitivity in a surrounding shell around the target location. On the other hand, the sensitivity of deep-focusing amplitude measurements peaks at the target location. The measurements have noise due to the stochastic excitation of the waves. In the case of a highly localized sound-speed perturbation, we find that the signal-to-noise ratio of deep-focusing amplitude measurements is higher than for deepfocusing travel-time measurements. These results obtained for a homogeneous medium, suggest that amplitude measurements may be used in local helioseismology in addition to the travel times.

In the main part of this thesis, we perform inversions of helioseismic travel times to infer the profile of the solar meridional flow. The observations cover two solar cycles from 1996 until 2019. Employing the constraint of mass conservation, we find that the solar meridional flow has a single-cell structure in each hemisphere: poleward at the surface and equatorward at the base of the convection zone with an amplitude of approximately 
$4 \mathrm{~m} / \mathrm{s}$ at latitude $45 \mathrm{deg}$. At the base of the convection zone, the velocity is equatorward with a functional form approximately given by $U_{\theta}=U_{b} \sin 2 \theta$, with $U_{b}=4.8 \pm 1.0 \mathrm{~m} / \mathrm{s}$ for cycle 23 and $U_{b}=3.6 \pm 1.0 \mathrm{~m} / \mathrm{s}$ for cycle 24 . The flow switches sign at a depth of about 0.79 solar radius. Confidence in the results is provided by the agreement between GONG and SOHO/MDI data during the period 2001-2011. According to a flux-transport dynamo model, the inferred meridional flow is able to explain the migration of sunspots towards the equator in each hemisphere.

The details of the inversion procedure and additional tests with synthetic data are presented in a complementary chapter. The inversions are tuned and validated using different test cases. We find that mass conservation is a necessary constraint to reconstruct the radial component of the meridional flow. A regularization term must also be introduced to avoid fast variations in latitude.

Finally, we discuss future developments in helioseismic inversions. Particularly promising are full-waveform inversions, which ought to provide improvements in both localization and noise levels. 


\section{Introduction}

\subsection{Solar meridional circulation}

The energy produced in the solar core by nuclear reactions is transported by radiation from the core to $0.7 R_{\odot}$, where $R_{\odot}=696 \mathrm{Mm}$ is the solar radius. In the outermost $30 \%$ of the solar interior, the thermal energy is predominantly transported by convection in the form of bulk fluid motions. The solar convection zone is highly stratified and dynamic.

At the surface, the Sun rotates with a period of approximately 25 days at the equator and 34 days at the poles. Thanks to helioseismology, we know that latitudinal rotation persists throughout the convection zone and that are regions of radial shear near the surface and at the base of the convection zone (e.g. Howe 2009).

An important component of motion in the solar convection zone is the meridional flow, which is the global (axisymmetric) poloidal component of the motion in the solar convection zone. Solar meridional circulation was first observed at the solar surface by Duvall (1979). He observed a poleward flow with a magnitude of about $20 \mathrm{~m} / \mathrm{s}$. Later on, other measurements confirmed that surface meridional flow is a poleward flow and peaks at mid-latitudes with an amplitude of $10-20 \mathrm{~m} / \mathrm{s}$ (e.g. Hathaway 1996, Ulrich 2010).

It is hypothesized that meridional circulation is driven by a slight imbalance between the non-conservative centrifugal and buoyancy forces (Kitchatinov 2016). The driving due to the centrifugal force arises because the solar rotation rate is not constant on cylinders aligned with the rotation axis. This drives a meridional flow in the sense required to produce a rotation rate which is constant on cylinders (e.g. Miesch et al. 2012). The fact that the rotation rate is not constant on cylinders is known from helioseismic observations and expected from theory. The competing term, related to buoyancy, is associated with an expected latitudinal entropy gradient. This results in a misalignment at surfaces of constant pressure and density, and drives a meridional flow which tries to align these surfaces. These two driving forces are large in the solar convection zone, and the observed meridional flow is thought to result from the small imbalance between the centrifugal and buoyancy forces (e.g. Kitchatinov 2016).

Meridional circulation is an essential ingredient in flux-transport dynamo models. Surface meridional flow transports magnetic flux to the poles to generate poloidal magnetic field (e.g. Cameron and Schüssler 2017)) and to start the next solar activity cycle. In single-cell geometries, the deep equatorward meridional flow plays a crucial role in the flux-transport dynamo models (e.g. Choudhuri et al. 1995): it transports the toroidal flux towards the equator and is responsible for the observed butterfly diagram at the surface (see e.g. review by Charbonneau (2010)). The butterfly diagram is a map of sunspot emergence latitudes versus solar-cycle phase. 

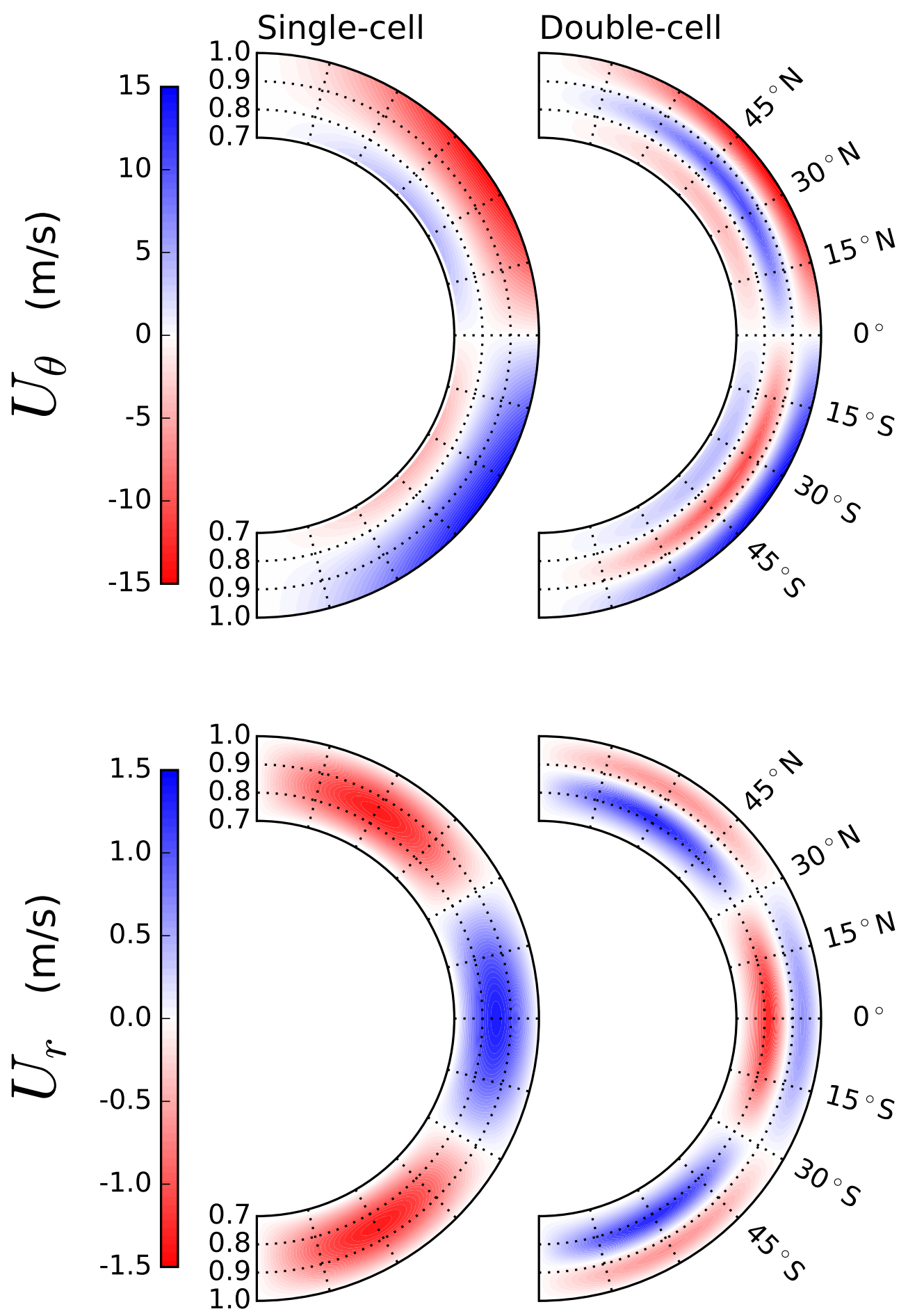

Figure 1.1: Synthetic single-cell and double-cell solar meridional flow models for the colatitudinal $U_{\theta}$ (top panels) and radial $U_{r}$ components (bottom panels) of the meridional flow circulating in the solar convection zone. Blue indicates southward and outward flows in the top and bottom panels, respectively. 
Numerical modeling has suggested both single-cell and double-cell geometries for solar meridional circulation (e.g. Featherstone and Miesch 2015, Pipin and Kosovichev 2018). Example single-cell and double-cell solar meridional flow models are shown in Figure 1.1. For the single-cell flow profile, the meridional flow near the bottom of the solar convection zone is equatorward. It is poleward in the case of the double-cell flow profile. Variations in magnitude and structure of the solar meridional flow can remarkably influence flux-transport dynamo models in terms of strength and length of the solar magnetic cycles (e.g. Featherstone and Miesch 2015).

There have been indications that the meridional flow may change with the phase of the solar cycle. For a large part, these variations are likely due to the presence of surface inflows around active regions (e.g. Gizon 2004, Gizon and Rempel 2008).

Meridional circulation is about one hundred times smaller in amplitude than the solar rotation. Thus it is a lot harder to measure. The near-surface meridional flow has been measured by different methods, including magnetic feature tracking (e.g. Hathaway and Rightmire 2010, Hathaway and Upton 2014), surface Doppler measurements (e.g. Hathaway 1996, Ulrich 2010), and local helioseismology (e.g. Patron et al. 1995, Braun and Fan 1998, Haber et al. 2002, Basu and Antia 2010). Giles et al. (1997) detected the subsurface meridional flow in the outer $4 \%$ of the Sun for the first time using time-distance helioseismology. Additional helioseismology measurements were provided by, e.g. Beck et al. (2002), Zhao and Kosovichev (2004), Zhao et al. (2014).

Helioseismic inferences of the meridional flow by different authors and different methods are not consistent in the deeper layers. In the first inversion results by Giles (2000), the meridional flow is inferred to be $3 \mathrm{~m} / \mathrm{s}$ equatorward at the base of the convection zone with a return flow occurring at depths below $0.80 R_{\odot}$. There were a few other attempts for inferring the solar meridional flow using time-distance helioseismic inversions. Zhao et al. (2013) used travel-time measurements from 2 years observations of Helioseismic and Magnetic Imager (HMI) onboard the Solar Dynamics Observatory (SDO/HMI: Scherrer et al. (2012), Schou et al. (2012)) to infer the solar meridional flow. They find a doublecell flow profile in each hemisphere in the radial direction with near-surface poleward flow with a speed of $15 \mathrm{~m} / \mathrm{s}$. Their analysis shows that the meridional flow is equatorward between $0.82 R_{\odot}$ and $0.91 R_{\odot}$. This means that the meridional flow is again poleward below $0.82 R_{\odot}$. Jackiewicz et al. (2015) and Böning et al. (2017) used travel-time measurements obtained from about 2 years of ground-based Global Oscillation Network Group (GONG: Harvey et al. (1996) ) data set and their results suggest a shallow return flow below $0.9 R_{\odot}$ and the results did not show a significant evidence of multi-cell flow structures. Using a mass conservation constraint in terms of the stream function, Rajaguru and Antia (2015) and Mandal et al. (2018) used antisymmetrized travel-time measurements obtained from 4 and 6 years data of SDO/HMI, respectively. Their results suggest single-cell meridional flow profiles in each hemisphere in the radial direction with return flows below the depths $0.77 R_{\odot}$ and $0.78 R_{\odot}$, respectively. As it is seen, there are strong controversies about the structure and magnitude of the solar meridional flow and thus more profound investigations need to be accomplished to have better insights about this component of motion in the solar convection zone. 


\subsection{Solar oscillations and helioseismology}

Leighton et al.(1962) first observed the fluctuations of the vertical velocities of the plasma on the solar surface that are oscillating with a period of about five minutes. An example of an image of solar oscillations in Doppler velocity, known as Dopplergram, is shown in Figure 1.2. Solar oscillations were hypothesized as the manifestations of standing acoustic waves (Ulrich 1970, Leibacher and Stein 1971). Deubner (1975) confirmed this interpretation by further observations of photospheric velocity field. It was confirmed by Deubner (1975) that the power in the oscillations is concentrated in ridges as shown in an example power spectrum of solar oscillations in Figure 1.3. As a matter of fact, some of the waves form standing waves as they are trapped in a resonant cavity formed by the Sun. This thus leads to an analysis of normal modes which are referred to different standing waves.

Each normal mode of oscillations can be described by three quantum numbers $n, l$, and $m$. Here, $n$ is the radial order that quantifies the number of nodes in the radial direction. $l$ is the harmonic degree and $m$ is the azimuthal order that are the numbers of node lines in the horizontal and longitudinal directions, respectively. The manifestation of solar oscillation modes can then be described as linear superposition of spherical harmonics at the surface. In Figure 1.3, the light ridges are due to the concentrated power in the oscillations that demonstrate the superposition of the normal modes of the Sun. More details on stellar oscillations can be found, e.g. in Christensen-Dalsgaard (2002).

solar oscillations are excited stochastically and it is not yet feasible to identify the sources of excitation in space or time. The near-surface turbulent convection in the solar interior is believed to be the driving mechanism of these normal modes (e.g. Goldreich and Keeley 1977, Houdek 2006, Houdek and Dupret 2015). These various modes of waves can be categorized by their restoring forces. In the solar convection zone, the predominant modes are pressure $(p)$ modes that are acoustic (sound) waves. The restoring force of these modes is pressure and they are also modified by gravity. The oscillatory power of $p$-modes peaks around $3 \mathrm{mHz}$. As sound speed is increasing inwards the solar interior, these waves get refracted as they penetrate in the solar interior and they return towards the surface when their horizontal phase speed is equal to the local sound speed (see e.g. Gizon et al. 2010), in which is called the lower turning point. The pressure modes are evanescent in the outer solar atmosphere and most of them get reflected near the solar surface due to the abrupt drop of density. The second type of modes are the surface gravity modes or fundamental $(f)$ modes. These modes only propagate near the solar surface and their restoring force is buoyancy and they are analogous to waves on the surface of oceans in Earth. Gravity $(g)$ modes are the third type of modes propagating in the deep solar interior, i.e. the radiative zone. The restoring force of $g$-modes is buoyancy (hence indirectly gravity). These lower-frequency modes are evanescent in the convection zone (where the stratification is unstable with respect to buoyancy) and thus have small amplitudes on the solar surface. Hence, $g$-modes are very difficult to be directly detected and there are only claims for their detection (e.g. Fossat et al. 2017) but there is still no consensus about the detection of solar $g$-modes (e.g. Appourchaux et al. 2010, Schunker et al. 2018).

Helioseismology takes advantage of continuous monitoring of oscillations on the solar surface and interprets the information encoded in the solar oscillations to probe the 


\section{Single Dopplergram}

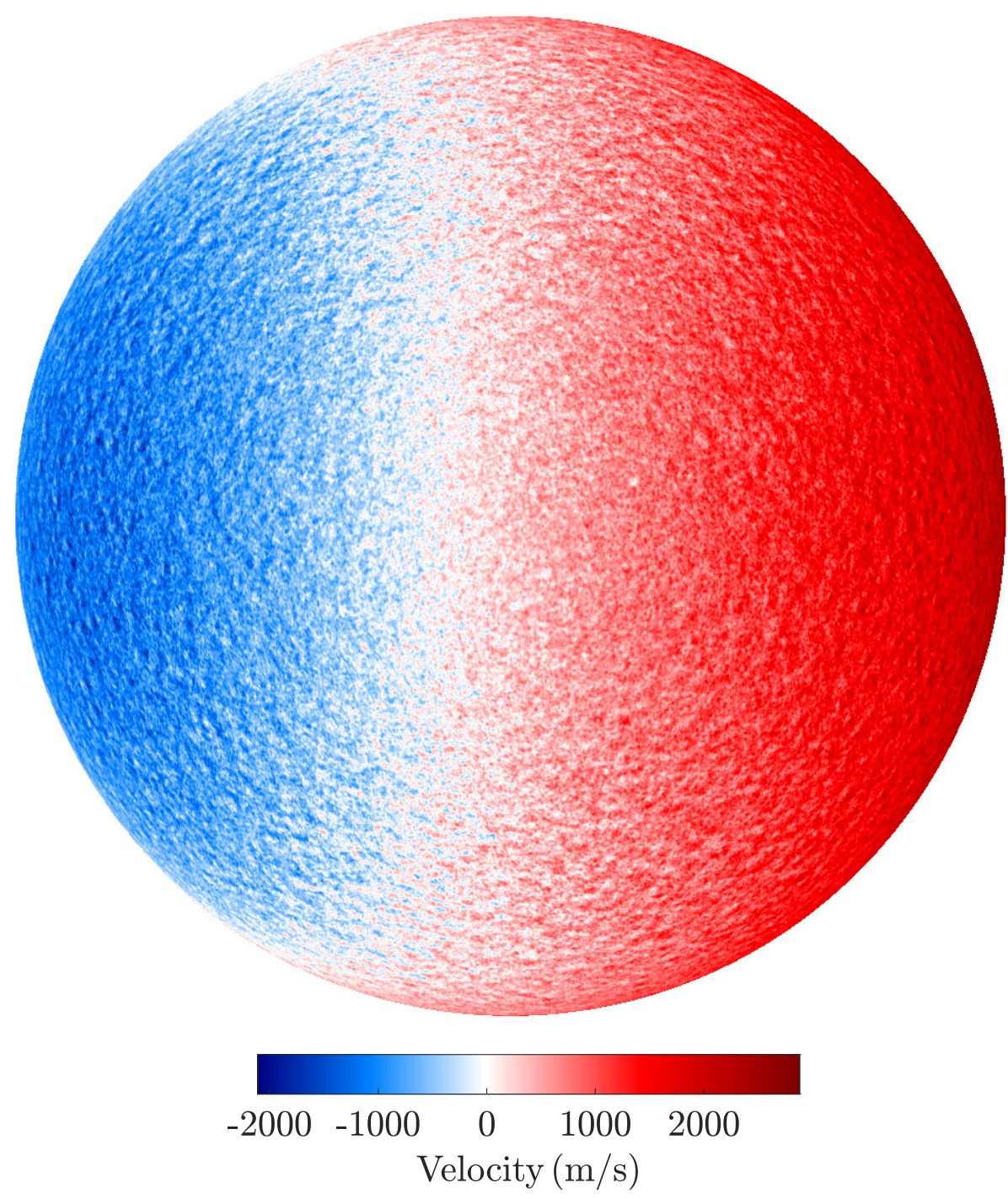

Figure 1.2: A single SOHO/MDI Dopplergram of the solar disk taken on 15 June 1996. Positive and negative values indicate velocities away from the observer (redshift) and towards the observer (blueshift), respectively. The gradient from east to west (left to right) is due to the solar rotation. Courtesy of the SOHO/MDI consortium. SOHO is a project of international cooperation between ESA and NASA.

solar interior. Based on the spatial scales of the solar structure to be targeted, helioseismology is classified into two major sub-fields, global and local. Global helioseismology (e.g. Christensen-Dalsgaard 2002, Broomhall et al. 2014) studies the normal modes and exploits directly the eigenfrequencies (the natural resonant frequencies of the normal modes) of these modes. Global helioseismology provides two-dimensional profiles and searches for helioseismic models by matching the model and observational oscillation frequencies to constrain solar physical properties on global scales such as sound speed (e.g. Christensen-Dalsgaard et al. 1985) or solar differential rotation (e.g. Schou et al. 1998, Thompson et al. 2003). Local helioseismology is referred to a set of techniques that use 


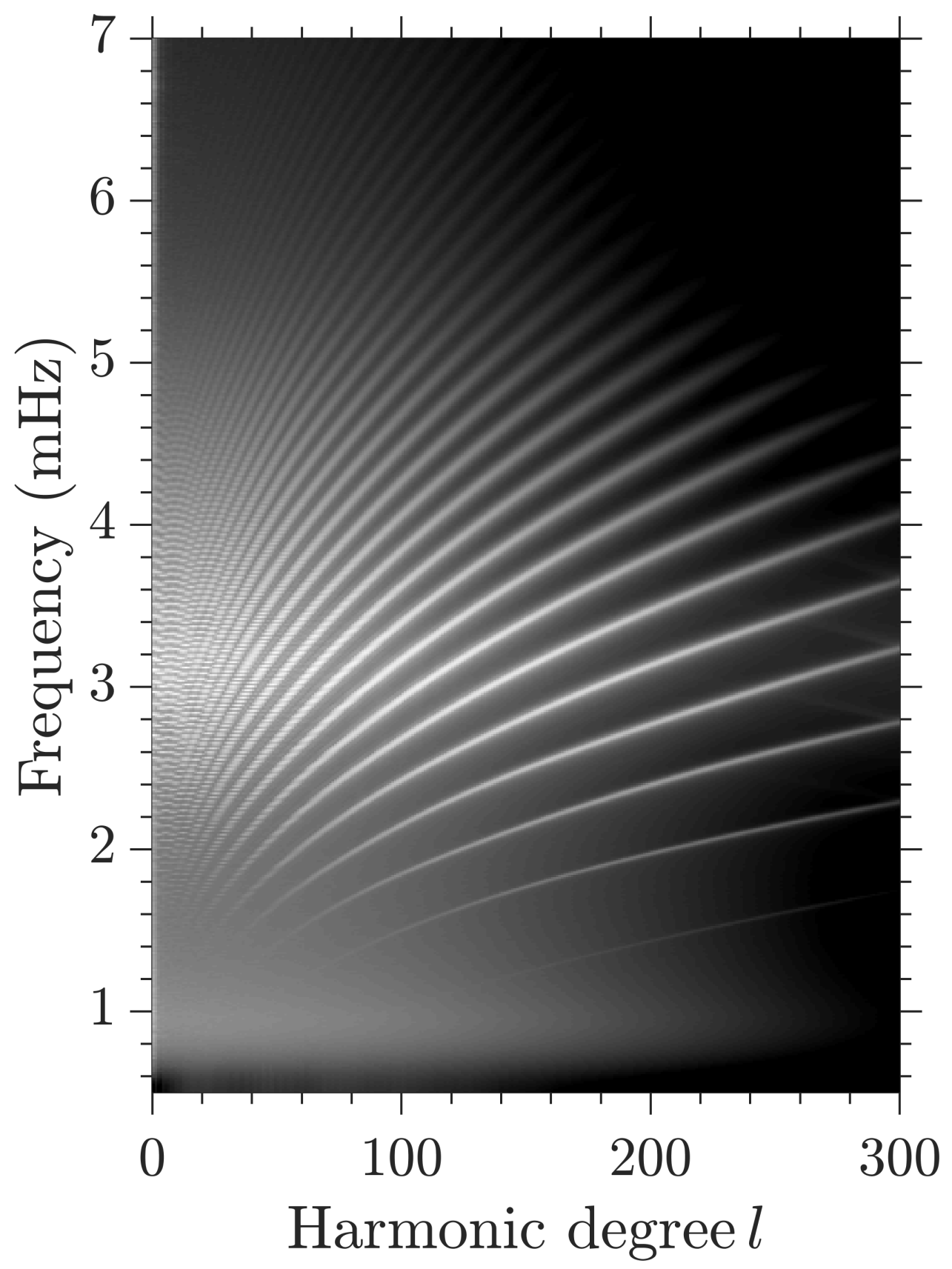

Figure 1.3: Example $m$-averaged power spectrum of solar oscillations obtained for one month of medium- $l$ data from SOHO/MDI as a function of harmonic degree $l$ (or equivalently horizontal wavenumber $k_{h} \approx l / R_{\odot}$ ) and frequency. Light regions indicate areas of high wave power. The power spectrum data courtesy of Zhi-Chao Liang.

local frequencies of modes and the cross-covariance function between the oscillation signals observed at any pair of points on the solar surface to probe the solar interior in local scales and in three dimensions. Local helioseismology includes various methods such as Fourier-Hankel method (Braun et al. 1987), Ring-diagram analysis (Hill 1988), helioseis- 
mic holography (Lindsey and Braun 1990), and time-distance helioseismology (Duvall et al. 1993). Explaining each individual method in local helioseismology is beyond the scope of this dissertation but thorough reviews are provided by Gizon and Birch (2005) and Gizon et al. (2010). As this thesis utilizes time-distance helioseismology, I only focus on this technique.

Local helioseismology mostly uses Dopplergrams as time series of solar observations (see a single frame of Dopplergram in Figure 1.2). The starting point in time-distance helioseismology is to compute the cross-covariance function between the oscillation signals observed at two locations on the solar surface. The temporal cross-covariance between the Doppler signals observed at two locations $\boldsymbol{r}_{1}$ and $\boldsymbol{r}_{2}$ on the solar surface is defined as

$$
C\left(\boldsymbol{r}_{1}, \boldsymbol{r}_{2}, t\right)=\frac{1}{T} \int_{-T / 2}^{T / 2} \Phi\left(\boldsymbol{r}_{1}, t^{\prime}\right) \Phi\left(\boldsymbol{r}_{2}, t^{\prime}+t\right) \mathrm{d} t^{\prime},
$$

where $\Phi\left(\boldsymbol{r}_{1}, t^{\prime}\right)$ denotes the observed Doppler signal at time $t^{\prime}$ at surface location $\boldsymbol{r}_{1}$, $\Phi\left(\boldsymbol{r}_{2}, t^{\prime}\right)$ denotes the observed Doppler signal at time $t^{\prime}$ at surface location $\boldsymbol{r}_{2}, t$ is the correlation time lag, and $T$ is the duration of observation (see Figure 1.4). The crosscovariance is considered as a solar seismogram because we can extract information about travel times and amplitudes of the wave packets traveling between any two surface locations. The cross-covariance is also referred to as "full waveform" specially in the geophysics literature (e.g. Fichtner 2010). It is worth mentioning that the cross-covariance function is related to the local power spectrum under the assumption of invariant horizontal medium: the cross-covariance function is obtained by the inverse Fourier transform of the local power spectrum (Gizon and Birch 2002, Gizon et al. 2010).

The cross-covariance and any data products extracted from the cross-covariance (e.g. wave travel times) are sensitive to the local physical conditions (structure and flows) where the wave packets propagating between any two surface locations. For instance, the waves travel slower against a flow than along a flow. In other words, perturbations in the solar subsurface physical properties with respect to a reference solar model result in perturbations in the helioseismic measurements. Hence, helioseismic measurements contain information about the solar subsurface physical properties. In time-distance helioseismology (Duvall et al. 1993), one measures travel times of solar waves traveling between any two locations on the solar surface to probe the solar subsurface structure and flows. This procedure includes measuring the wave travel times from the cross-covariance function and inverting the wave travel times to infer the physical properties in the solar interior (the inverse problem). This interpretation of the travel times also requires computation of the propagation of the waves through a solar model (the forward problem).

Due to the stochastic excitation of solar oscillations, helioseismic measurements like cross-covariance are very noisy and spatial and temporal averages are usually carried out in order to reduce the random noise. For instance, Duvall et al. (1993) suggested an averaging scheme as "point-to-annulus geometry" that the cross-covariance is computed between a point and a concentric annulus. As another example, Duvall et al. (1997) considered a "point-to-quadrant geometry" for spatial averaging that the cross-covariance is computed between a point and quadrants of arc. An "arc-to-arc" geometry is usually used for probing the subsurface meridional flow (e.g. Liang et al. 2017). The cross-covariance function is usually computed utilizing one of these spatial averaging schemes to improve the signal-to-noise ratio. From the cross-covariance, travel times can be measured by fit- 

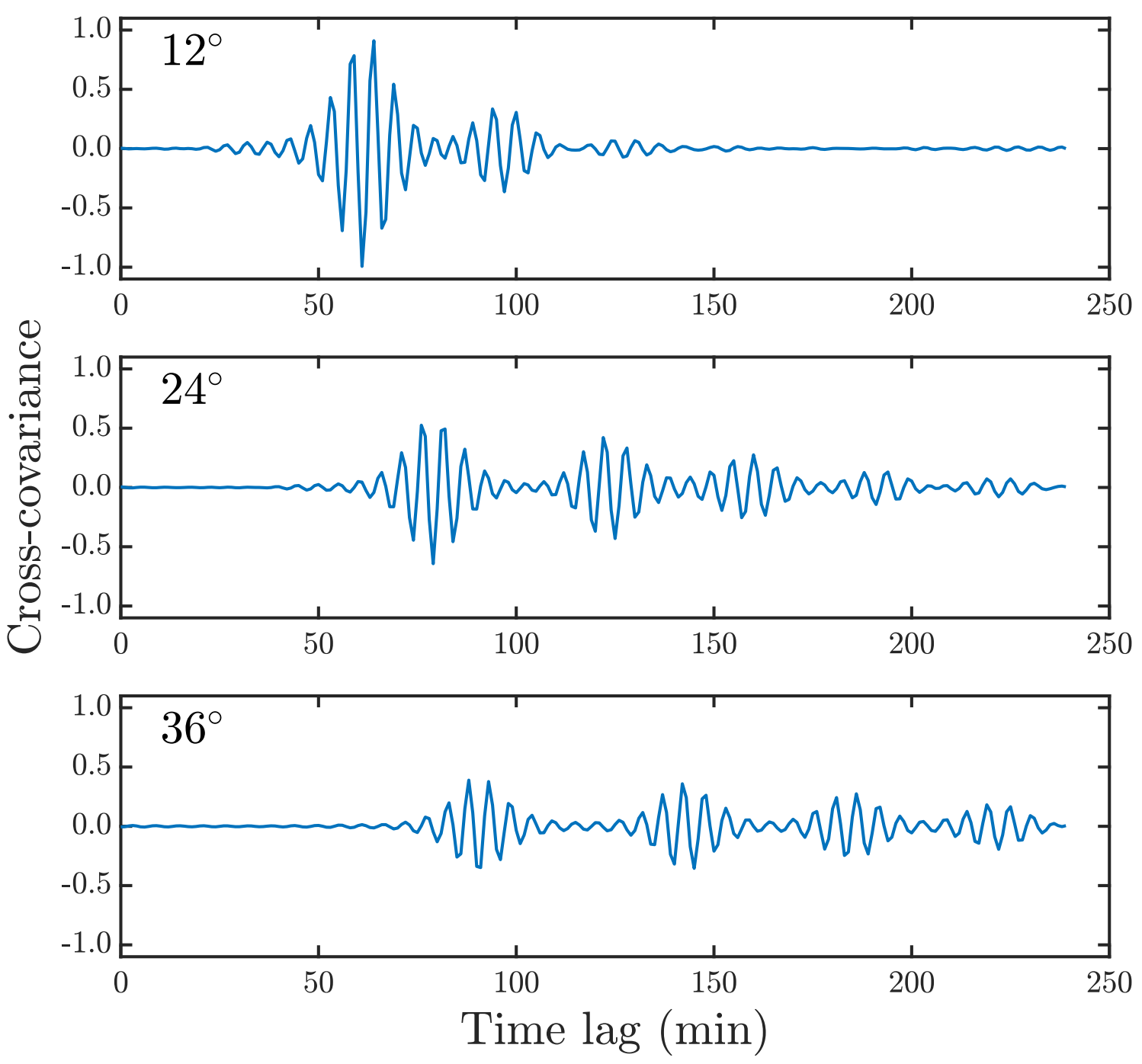

Figure 1.4: Normalized SOHO/MDI longitudinally averaged cross-covariance as a function of time lag obtained from Doppler observations for three different pairs of points separated by angular distances $12^{\circ}, 24^{\circ}$, and $36^{\circ}$.

ting a Gabor wavelet (Duvall et al. 1997) or by a convenient one-parameter fit (Gizon and Birch 2004) to the first-skip wave packets. Hence, the resulting (phase) travel-time measurements would have higher signal-to-noise ratio as they are temporal-averaged quantities of cross-covariance measurements. However, the higher signal-to-noise ratio costs us to lose some information contained in the cross-covariance.

The full-waveform approach (using the cross-covariance measurements) is broadly used in geophysics (see e.g. review by Virieux and Operto 2009) and has been rarely used in helioseismology (e.g. Hanasoge and Tromp 2014). However, travel-time measurements have been widely used in helioseismology (e.g. Gizon and Birch 2005, Böning et al.|2017, Mandal et al. 2018). In addition to the cross-covariance (full waveform) and travel-time measurements, the cross-covariance amplitude may also be used in helioseismic investigations. The amplitudes of the wave packets traveling between any two surface points provide additional information than travel times. For instance, measurements of cross- 
covariance amplitude are a better set of input data to study the attenuation of the waves (e.g. Dalton and Ekström 2006, Nagashima et al. 2017). Therefore, different types of input data can be used in helioseismic studies based on the availability of the measurements and the physical property of interest.

\subsection{The forward and inverse problems}

Many solar features such as meridional flow, supergranulation and torsional oscillations can be approximated as weak perturbations to a reference solar model. In this manner, helioseismic measurements can be linearly linked to the perturbations in the solar physical properties with respect to a reference solar model. In time-distance helioseismology, a linear forward problem is defined as

$$
d_{i}=\sum_{\beta=1}^{P} \int_{\odot} K_{i}^{\beta}(\boldsymbol{r}) \delta q_{\beta}(\boldsymbol{r}) \mathrm{d}^{3} \boldsymbol{r}+n_{i},
$$

where $\delta q_{\beta}$ denotes a set of $P$ perturbations, indexed by $\beta$, in the various solar physical properties (e.g. pressure $p$, density $\rho$, sound speed $c$, the components of the vector flow $\left.\boldsymbol{U}=\left(U_{r}, U_{\theta}, U_{\varphi}\right)\right)$ with respect to a reference solar model. The integration variable $\boldsymbol{r}$ is a 3D position vector $\boldsymbol{r}=(r, \theta, \varphi)$ with radius $r$, colatitude $\theta$, and longitude $\varphi$ in spherical-polar coordinates. The volume integral is an integration over the whole Sun. In Equation 1.2, $d_{i}$ is a set of helioseismic measurements (e.g. travel-time perturbations $\delta \tau_{i}$ ), $n_{i}$ are the corresponding noise of the measurements, and $K_{i}^{\beta}$ are the sensitivity functions (also called sensitivity kernels). The index $i=\left(\boldsymbol{r}_{i}, \boldsymbol{r}_{i}^{\prime}\right)$ is associated with each helioseismic measurement corresponding to a pair of points on the solar surface where $\boldsymbol{r}_{i}=\left(R_{\odot}, \theta_{i}, \varphi_{i}\right)$ and $\boldsymbol{r}_{i}^{\prime}=\left(R_{\odot}, \theta_{i}^{\prime}, \varphi_{i}^{\prime}\right)$.

The main ingredient of the forward problem in helioseismology is sensitivity kernels. Sensitivity kernels quantify the responses of the helioseismic measurements to the perturbations in the solar interior. To compute the sensitivity kernels, one usually uses the ray approximation (e.g. Kosovichev 1996, Kosovichev and Duvall 1997) or the first Born approximation (e.g. Gizon and Birch 2002). The ray approximation is a high-frequency approximation where the resulting sensitivity kernels are only sensitive along a geometrical ray path. On the other hand, the first Born approximation models the wave field under a single-scattering approximation. Hence, the Born sensitivity kernels include the finitewavelength effects and have sensitivities away from local ray paths as well. The Born sensitivity kernels depend on the Green's function associated to a wave equation (see e.g. Gizon et al. (2017)) with solar background and on the cross-covariance. Green's function is the response of the medium to an impulsive source of excitation. This work follows the framework of Gizon et al. (2017), where the Green's function is associated to a scalar wave equation and the expectation value of the cross-covariance is directly obtained from the imaginary part of the frequency-domain Green's function.

In order to infer the solar subsurface physical properties from the helioseismic measurements, one needs to solve an inverse problem. In linear inversions, we seek for estimated solution of $\delta q_{\mu}\left(\boldsymbol{r}_{0}\right)$ denoted by $\delta \hat{q}_{\mu}\left(\boldsymbol{r}_{0}\right)$ at each target point $\boldsymbol{r}_{0}=\left(r_{0}, \theta_{0}, \varphi_{0}\right)$ as linear 
combination of the input data (see Equation 1.2) such that

$$
\begin{aligned}
\delta \hat{q}_{\mu}\left(\boldsymbol{r}_{0}\right) & =\sum_{i} w_{i}^{\mu}\left(\boldsymbol{r}_{0}\right) d_{i} \\
& =\int_{\odot} \mathcal{K}_{\mu}^{\mu}\left(\boldsymbol{r}_{0}, \boldsymbol{r}\right) \delta q_{\mu}(\boldsymbol{r}) \mathrm{d}^{3} \boldsymbol{r}+\int_{\odot} \sum_{\beta=1, \beta \neq \mu}^{P} \mathcal{K}_{\beta}^{\mu}\left(\boldsymbol{r}_{0}, \boldsymbol{r}\right) \delta q_{\beta}(\boldsymbol{r}) \mathrm{d}^{3} \boldsymbol{r}+\sum_{i} w_{i}^{\mu}\left(\boldsymbol{r}_{0}\right) n_{i},
\end{aligned}
$$

where

$$
\mathcal{K}_{\beta}^{\mu}\left(\boldsymbol{r}_{0}, \boldsymbol{r}\right)=\sum_{i} w_{i}^{\mu}\left(\boldsymbol{r}_{0}\right) K_{i}^{\beta}(\boldsymbol{r})
$$

and the $w_{i}^{\mu}$ are weights to be determined. The averaging kernels $\mathcal{K}_{\mu}^{\mu}\left(\boldsymbol{r}_{0}, \boldsymbol{r}\right)$, also called resolution kernels, show a weighted average of the sensitivity kernels at each target location and it is desirable that they are spatially localized near the target $\boldsymbol{r}=\boldsymbol{r}_{0}$. The cross-talk averaging kernels $\mathcal{K}_{\beta}^{\mu}\left(\boldsymbol{r}_{0}, \boldsymbol{r}\right)$ with $\beta \neq \mu$ show how much other perturbations $\beta \neq \mu$ leak into $\delta \hat{q}_{\mu}\left(\boldsymbol{r}_{0}\right)$. One would ideally like the cross-talk averaging kernels to be zero. The last term in Equation 1.3 represents the propagation of random errors from the helioseismic input data into the inverted solution $\delta \hat{q}_{\mu}\left(\boldsymbol{r}_{0}\right)$.

To solve the inverse problem, the problem is discretized by decomposing the unknowns into linear combinations of basis functions as

$$
\delta q_{\beta}(\boldsymbol{r})=\sum_{j} a_{j}^{\beta} \phi_{j}(\boldsymbol{r}), \quad \beta \in[1, P]
$$

where $a_{j}^{\beta}$ are the coefficients corresponding to each physical property of interest, labeled by $\beta$, to be determined and $\phi_{j}(\boldsymbol{r})$ are the basis functions introduced by

$$
\phi_{j}(\boldsymbol{r})=\phi_{k}^{r}(r) \phi_{h}^{\theta}(\theta) \phi_{f}^{\varphi}(\varphi), \quad j:=(k, h, f) .
$$

$\phi_{k}^{r}, \phi_{h}^{\theta}$, and $\phi_{f}^{\varphi}$ are the basis functions in the radial, colatitudinal and azimuthal directions, respectively. We consider the forward problem (in Equation 1.2) in matrix form

$$
\boldsymbol{d}=K \boldsymbol{q}+\boldsymbol{n}
$$

where $\boldsymbol{d}$ is a vector of input data, $\boldsymbol{n}$ is the corresponding noise vector, and $\boldsymbol{q}$ is a vector of unknown coefficients of all the physical properties of interest

$$
\boldsymbol{q}=\left[\begin{array}{c}
\boldsymbol{a}^{1} \\
\vdots \\
\vdots \\
\boldsymbol{a}^{P}
\end{array}\right]
$$

Note that for each physical property of interest, a vector such $\boldsymbol{a}^{\beta}$ contains the coefficients introduced in Equation 1.5. The kernels for different perturbations and measurements are written in a matrix of kernel coefficients $K=\left[\tilde{K}^{1} \cdots \cdots \tilde{K}^{P}\right]$ where elements of $\tilde{K}^{\beta}$ are obtained by projecting the sensitivity kernels onto the basis functions:

$$
\tilde{K}_{i j}^{\beta}=\int_{\odot} K_{i}^{\beta}(\boldsymbol{r}) \phi_{j}(\boldsymbol{r}) \mathrm{d}^{3} \boldsymbol{r}, \quad \beta \in[1, P] .
$$


Following the approach in Equation 1.3 , we seek for a solution vector $\hat{\boldsymbol{q}}$ that estimates the vector of the unknown coefficients $\boldsymbol{q}$ such that

$$
\hat{q}=\mathcal{W},
$$

where each row of the weight matrix $\mathcal{W}$ is associated with the corresponding coefficients in Equation 1.8. In this thesis, we use the regularized least squares (RLS) method (also called Tikhonov regularization) (e.g. Kosovichev 1996) to invert the helioseismic measurements for the physical property of interest. RLS minimizes the misfit of the forward model to the observational data plus a regularization term. Hence, we seek the vector of the unknown coefficients $\boldsymbol{q}$ that minimizes

$$
\left\|\Lambda^{-1 / 2}(K \boldsymbol{q}-\boldsymbol{d})\right\|^{2}+\alpha\|D \boldsymbol{q}\|^{2} .
$$

The solution $\hat{\boldsymbol{q}}$ is obtained from Equation 1.10 where

$$
\mathcal{W}=\left(K^{H} \Lambda^{-1} K+\alpha D^{H} D\right)^{-1} K^{H} \Lambda^{-1},
$$

where the superscript $H$ denotes the Hermitian conjugate. In Equation 1.11, $\|\cdot\|$ is the discrete $L^{2}$ norm, $\alpha$ is a regularization parameter, $D$ is a regularization matrix, and $\Lambda$ is the noise covariance matrix of the helioseismic measurements, which can be estimated from the helioseismic data or from a noise model (e.g. Gizon and Birch 2004, Fournier et al. 2014). The regularization term $\alpha\|D \boldsymbol{q}\|^{2}$ is a penalty term that reduces the propagation of the noise from the helioseismic measurements. The optimal weight matrix in Equation 1.12 is obtained by choosing an optimal regularization parameter. For this choice, the regularization parameter $\alpha$ is tuned to obtain the best trade-off between bias and noise in the estimated solution. By having the optimal weight matrix in hand, the coefficients for each physical property can be estimated. With respect to Equation 1.5 , the estimated solution of $\delta q_{\beta}\left(\boldsymbol{r}_{0}\right)$ denoted by $\delta \hat{q}_{\beta}\left(\boldsymbol{r}_{0}\right)$ at each chosen target point $\boldsymbol{r}_{0}$ is thus written as

$$
\delta \hat{q}_{\beta}\left(\boldsymbol{r}_{0}\right)=\sum_{j} \hat{a}_{j}^{\beta} \phi_{j}\left(\boldsymbol{r}_{0}\right), \quad \beta \in[1, P],
$$

where $\hat{a}_{j}^{\beta}$ are the estimated coefficients of $a_{j}^{\beta}$ introduced in Equation 1.5 . The variance of the estimated solution at each target location is obtained by noticing that for each vector of $\boldsymbol{a}^{\beta}$, there exists a matrix $V_{\beta}$ (which is a sub-matrix of $\boldsymbol{W}$ ) such that $\boldsymbol{a}^{\beta}=V_{\beta} \boldsymbol{d}$. Hence, the variance of the estimated solution at each target location is given by

$$
\operatorname{Var}\left(\delta \hat{q}_{\beta}\left(\boldsymbol{r}_{0}\right)\right)=\phi\left(\boldsymbol{r}_{0}\right)^{H} V_{\beta} \Lambda V_{\beta}^{H} \phi\left(\boldsymbol{r}_{0}\right), \quad \beta \in[1, P],
$$

where $\phi\left(\boldsymbol{r}_{0}\right)$ denotes the vector of basis functions.

There are other inverse methods such as optimally localized averages (OLA) (e.g. Backus and Gilbert 1968), subtractive optimally localized averaging (SOLA, also known as method of approximate inverse in mathematics) (e.g. Pijpers and Thompson 1994, Jackiewicz et al. 2012), or Pinsker estimator (e.g. Fournier et al. 2016). SOLA is a subclass of the OLA methods that has been used extensively in helioseismology. The idea of SOLA method is to minimize the distance between the averaging kernel at each target point and a target function. The Pinsker method is a statistical linear optimal estimator that provides solutions by defining a minimax linear risk. 


\subsection{Contents of this thesis}

The main part of this thesis focuses on the helioseismic inversions of wave travel times to infer the solar meridional flow and the corresponding tests to tune and validate the helioseismic inversions. The thesis also contains a study comparing the deep-focusing travel-time and cross-covariance amplitude measurements for sound-speed perturbations with respect to a homogeneous medium. Additionally, the thesis presents an initial comparison of travel-time and full-waveform inversions in time-distance helioseismology.

In chapter 2, we compare travel-time and amplitude measurements for the deepfocusing time-distance helioseismology. These measurements are extracted from the deep-focusing cross-covariance functions. We consider a homogeneous medium and compute the deep-focusing sensitivity kernels for sound-speed perturbations. We find that the spatial sensitivity of travel-time deep-focusing measurements is zero at the target location and is maximum in a surrounding shell around the target location. Unlike the deep-focusing travel-time measurements, the sensitivity of deep-focusing amplitude measurements is maximum at the target location. We perform a signal-to-noise analysis because the measurements have noise due to the stochastic excitation of the waves. We consider two different types of sound-speed perturbations. In one of the cases that we consider a highly localized sound-speed perturbation, we find that the signal-to-noise ratio is higher for the deep-focusing amplitude measurements than for the deep-focusing travel-time measurements.

In chapter 3, the main scientific contribution of this thesis is presented which is the inferences of the solar meridional flow. Inversions of helioseismic travel times are performed to infer the solar meridional flow over the solar cycles 23 and 24 (1996-2019). We find a single-cell structure in each hemisphere for the solar meridional with a return flow occurring at depths below about $0.79 R_{\odot}$. By performing flux-transport dynamo simulations, we find that the inferred meridional flow is able to explain the equatorial migration of sunspots in each hemisphere.

In chapter 4, we perform helioseismic inversions for the solar meridional flow using synthetic travel times. As a complement to chapter 3, this section attempts to provide a clear procedure on tuning and validation of the helioseismic inversions. For this aim, different test cases are considered and both single-cell and double-cell meridional flow models are used. The results show that the constraint of mass conservation is necessary to reconstruct the radial component of the meridional flow. Additional tests are performed regarding the regularization term, the boundary constraint at the base of the convection zone, and the effect of the large-distance travel times on the inversions.

In chapter 5, we study full-waveform inversion as a new inversion approach in this thesis. At fixed noise level, the spatial resolutions of inversions for flows are compared for different choices of helioseismic observables including full waveform and travel times. The main conclusions obtained in the thesis and a few suggestions for future work are also presented in this chapter. 


\title{
2 Comparison of travel-time and amplitude measurements for deep-focusing time-distance helioseismology ${ }^{1}$
}

\begin{abstract}
The purpose of deep-focusing time-distance helioseismology is to construct seismic measurements that have a high sensitivity to the physical conditions at a desired target point in the solar interior. With this technique, pairs of points on the solar surface are chosen such that acoustic ray paths intersect at this target (focus) point. Considering acoustic waves in a homogeneous medium, we compare travel-time and amplitude measurements extracted from the deep-focusing cross-covariance functions. Using a single-scattering approximation, we find that the spatial sensitivity of deep-focusing travel times to sound-speed perturbations is zero at the target location and maximum in a surrounding shell. This is unlike the deep-focusing amplitude measurements, which have maximum sensitivity at the target point. We compare the signal-to-noise ratio for travel-time and amplitude measurements for different types of sound-speed perturbations, under the assumption that noise is solely due to the random excitation of the waves. We find that, for highly localized perturbations in sound speed, the signal-to-noise ratio is higher for amplitude measurements than for travel-time measurements. We conclude that amplitude measurements are a useful complement to travel-time measurements in time-distance helioseismology.
\end{abstract}

\subsection{Introduction}

Time-distance helioseismology (Duvall et al. 1993) is a branch of local helioseismology (e.g. Gizon and Birch 2005) that aims at probing the complex subsurface structures of the solar interior. The time-distance method measures the travel times of acoustic waves between any pair of points on the solar surface from the cross-covariance function of

\footnotetext{
${ }^{1}$ This chapter reproduces the article Comparison of travel-time and amplitude measurements for deepfocusing time-distance helioseismology by M. Pourabdian, D. Fournier, and L. Gizon, published in Solar Physics 293, 66 (2018), DOI https://doi.org/10.1007/s11207-018-1283-8. Reproduced under the terms of the Creative Commons Attribution 4.0 International License (http://creativecommons. org/licenses/by/4.0/). Contribution statement: MP performed research, accomplished the numerical computations, and wrote the paper with contributions from DF and LG.
} 
2 Comparison of travel-time and amplitude measurements for deep-focusing time-distance helioseismology

the observed oscillation signals. Seismic travel times contain information about the local physical properties of the medium and have thus been broadly used in helioseismology (e.g. Gizon and Birch 2002, Birch et al. 2004, Gizon et al. 2010).

A consistent issue with local helioseismology is the signal-to-noise ratio. When examining near-surface structures such as supergranular flows (Duvall et al. 1996, Langfellner et al. 2015), averaging is typically performed around an annulus, where the crosscovariance is calculated between the center point and the average signal in the annulus. This technique is highly sensitive to near-surface perturbations. To probe greater depths, one would seek a different averaging technique that has peak sensitivity at any chosen target depth. Such a technique is known as deep-focusing and was first described by Duvall (1995), who outlined a procedure in which points on the surface are chosen such that a large number of connecting ray paths intersect at the target (focus) point, with the expectation that sensitivity is large near the target depth. The deep-focusing timedistance technique has been employed to study the meridional flow in the solar interior (e.g. Hartlep et al. 2013, Zhao et al. 2013) and sunspot structure (e.g. Moradi and Hanasoge 2010). Jensen (2001) investigated the application of the deep-focusing method to improve inversions for large sunspots. Using the Rytov approximation, he found sensitivity in a shell around the target point but zero sensitivity at the target point, consistent with wavefront healing seen in the Born approximation in geophysics and helioseismology (Liang et al. 2013). To resolve this drawback, Hughes et al. (2007) suggested an optimized technique for deep focusing that allocates weightings for each measurement. They obtained improvements in the results by considering travel-time measurements of synthetic experiments.

In addition to the travel times, the cross-covariance function contains additional information that may be of use to helioseismology. For instance, in terrestrial seismology cross-covariance amplitudes have been used to characterize seismic waves (e.g. Nolet et al. 2005). The importance of the amplitudes was examined by Dalton et al. (2014), who concluded that assumptions and simplifications in the measurement of surface-wave amplitudes affect the attenuation structure found through inversions. Moreover, Dahlen and Baig (2002) investigated the Fréchet sensitivity kernels using the geometrical ray approximation for travel-time and amplitude measurements. They found a maximum sensitivity along the point-to-point ray path when examining the amplitude of seismic-wave cross-correlation. In contrast to travel times, few studies have considered the amplitude measurements of the cross-covariance function in helioseismology. Liang et al. (2013) measured the spatial maps of wave travel times and amplitudes from the cross-covariance function of the wave field around a sunspot in the NOAO Active Region 9787. Using 2D ray theory, they observed an amplitude reduction that was attributed to the defocusing of wave energy by the fast-wave-speed perturbation in the sunspot. Recent work by Nagashima et al. (2017) described a linear procedure to measure the amplitude of the cross-covariance function of solar oscillations. This linear relation between the crosscovariance function and the amplitude allows the derivation of Born sensitivity kernels using the procedure of Gizon and Birch (2002), which provides a straightforward interpretation for the amplitude measurements.

The deep-focusing time-distance technique using amplitude measurements is lacking in time-distance helioseismology. Furthermore, the deep-focusing analysis has been considered only using the ray theory, which is a high-frequency approximation and does not 
take into account finite-wavelength effects. As a result, the ray approximation may be inaccurate for amplitude calculations (e.g. Tong et al. 1998). In this study, we use the deep-focusing time-distance technique to compare signal and noise for travel-time and amplitude measurements under the Born approximation. Section 2.2 describes the definition of travel-time and amplitude measurements and explains the deep-focusing technique and the noise model. The setup and derivation of sensitivity kernels are explained in Section 2.3 and the results are presented in Section 2.4. Conclusions are given in Section 2.5 .

\subsection{Travel-time and amplitude measurements}

\subsubsection{Definitions}

In time-distance helioseismology, one uses the cross-covariance function between the oscillation signals observed at any two points $\left[\boldsymbol{r}_{1}\right.$ and $\left.\boldsymbol{r}_{2}\right]$ on the solar surface to recover the desired information within the relevant wave-field observable. In general, we observe the line-of-sight velocity $[\phi]$ and define the temporal cross-covariance function for surface locations $\boldsymbol{r}_{1}$ and $\boldsymbol{r}_{2}$ as

$$
C\left(\boldsymbol{r}_{1}, \boldsymbol{r}_{2}, t\right)=\frac{1}{T} \int_{-T / 2}^{T / 2} \phi\left(\boldsymbol{r}_{1}, t^{\prime}\right) \phi\left(\boldsymbol{r}_{2}, t^{\prime}+t\right) \mathrm{d} t^{\prime},
$$

where $t$ is the time lag and $T$ is the duration of observation. Considering small changes to a reference solar model, one can define the incremental travel time $[\delta \tau]$ and relative amplitude $[\delta a]$ between the observed $\left[C\left(\boldsymbol{r}_{1}, \boldsymbol{r}_{2} ; t\right)\right]$ and reference $\left[C_{0}\left(\boldsymbol{r}_{1}, \boldsymbol{r}_{2} ; t\right)\right]$ cross-covariances as

$$
\begin{aligned}
& \delta \tau\left(\boldsymbol{r}_{1}, \boldsymbol{r}_{2}\right)=\int_{-T / 2}^{T / 2} W_{\tau}\left(\boldsymbol{r}_{1}, \boldsymbol{r}_{2}, t\right) \delta C\left(\boldsymbol{r}_{1}, \boldsymbol{r}_{2}, t\right) \mathrm{d} t, \\
& \delta a\left(\boldsymbol{r}_{1}, \boldsymbol{r}_{2}\right)=\int_{-T / 2}^{T / 2} W_{a}\left(\boldsymbol{r}_{1}, \boldsymbol{r}_{2}, t\right) \delta C\left(\boldsymbol{r}_{1}, \boldsymbol{r}_{2}, t\right) \mathrm{d} t,
\end{aligned}
$$

where

$$
\delta C\left(\boldsymbol{r}_{1}, \boldsymbol{r}_{2}, t\right)=C\left(\boldsymbol{r}_{1}, \boldsymbol{r}_{2}, t\right)-C_{0}\left(\boldsymbol{r}_{1}, \boldsymbol{r}_{2}, t\right) .
$$

The above linear relations between the measurements and the cross-covariance function are specified via the weighting functions $[W]$ given by Nagashima et al. (2017):

$$
\begin{aligned}
& W_{\tau}(t)=-\frac{w(t) \partial_{t} C_{0}(t)}{\int_{-T / 2}^{T / 2} w\left(t^{\prime}\right)\left[\partial_{t^{\prime}} C_{0}\left(t^{\prime}\right)\right]^{2} \mathrm{~d} t^{\prime}}, \\
& W_{a}(t)=\frac{w(t) C_{0}(t)}{\int_{-T / 2}^{T / 2} w\left(t^{\prime}\right)\left[C_{0}\left(t^{\prime}\right)\right]^{2} \mathrm{~d} t^{\prime}},
\end{aligned}
$$

where $w(t)$ is a window function that may select the first-arrival wave packet. With this definition of the weighting function $\left[W_{a}\right]$ the relative amplitude $[\delta a]$ is dimensionless.

Throughout this article, we use $q$ to denote either the travel-time $[\tau]$ or the amplitude measurement $[a]$. Using this compact notation, we write

$$
\delta q\left(\boldsymbol{r}_{1}, \boldsymbol{r}_{2}\right)=\int_{-T / 2}^{T / 2} W_{q}\left(\boldsymbol{r}_{1}, \boldsymbol{r}_{2}, t\right) \delta C\left(\boldsymbol{r}_{1}, \boldsymbol{r}_{2}, t\right) \mathrm{d} t, \quad \text { for } q \in\{\tau, a\} .
$$




\subsubsection{Deep-focusing averages}

The basic idea of the deep-focusing technique is to obtain high sensitivity to a physical quantity by focusing at a given target point. To do so, we consider a set of pairs of points on the solar surface such that the ray paths (straight lines for a homogeneous medium) intersect at a chosen target point. As an example, Figure 2.1 illustrates how these pairs of points could be distributed on the surface of the near-side of the Sun. In a solar case, the ray paths would be curved due to the sound-speed stratification.

For any desired target point $\left[\boldsymbol{r}_{t}\right]$ in the solar interior, we define the averaged travel-time and amplitude perturbations as

$$
\overline{\delta q}\left(\boldsymbol{r}_{t}\right)=\frac{1}{N} \sum_{i=1}^{N} \delta q_{i}\left(\boldsymbol{r}_{t}\right), \quad q \in\{\tau, a\},
$$

where $\delta q_{i}\left(\boldsymbol{r}_{t}\right)$ represents the point-to-point measurement between the points $\boldsymbol{r}_{i}$ and $\boldsymbol{r}_{i}^{\prime}$ chosen such that the ray path intersects at the focus point $\boldsymbol{r}_{t}$,

$$
\delta q_{i}\left(\boldsymbol{r}_{t}\right)=\delta q\left(\boldsymbol{r}_{i}, \boldsymbol{r}_{i}^{\prime}\right) .
$$

The observations points $\boldsymbol{r}_{i}$ and $\boldsymbol{r}_{i}^{\prime}$ are on a sphere of radius $\mathrm{R}_{\odot}$ and have coordinates $\boldsymbol{r}_{i}=$ $\left(\mathrm{R}_{\odot}, \theta_{i}, \phi_{i}\right)$ and $\boldsymbol{r}_{i}^{\prime}=\left(\mathrm{R}_{\odot}, \theta_{i}^{\prime}, \phi_{i}^{\prime}\right)$ in the spherical-polar coordinate system whose polar axis contains the target point (depicted in Figure 2.1). The index $i$ spans $[1, N]$, where $N=$ $N_{\theta} N_{\phi}$ is the total number of pairs of points, with $N_{\theta}$ the number of colatitudes and $N_{\phi}$ the number of longitudes. Each index $i$ is associated with a pair of indices $\left(i_{\theta}, i_{\phi}\right) \in\left[1, N_{\theta}\right] \times$ $\left[1, N_{\phi}\right]$, where the first index refers to the colatitudes $\theta_{i_{\theta}}$ and $\theta_{i_{\theta}}^{\prime}=\Delta-\theta_{i_{\theta}}$ (where $\Delta$ is the colatitude difference between the two observation points in a pair) and the second index refers to the uniformly-spaced azimuths $\phi_{i_{\phi}}=2 \pi\left(i_{\phi}-1\right) / N_{\phi}$ and $\phi_{i_{\phi}}^{\prime}=\phi_{i_{\phi}}+\pi$. The range of colatitudes $\theta_{1} \leq \theta \leq \theta_{N_{\theta}}$ defines the extent of the pupil. Choosing a maximum value $\theta_{N_{\theta}}=$ $65^{\circ}$, the value of $\theta_{1}$ then depends on the target depth. At a fixed longitude, the colatitudes of the points within the pupil are chosen such that the angle between neighboring ray paths is uniform.

\subsubsection{Noise model}

Here we describe the noise in the averaged measurements for travel time and amplitude. Random noise in helioseismology is due to the stochastic excitation of acoustic waves by turbulent convection. The noise model developed by Gizon and Birch (2004) is based on the reasonable assumption that the reference wave field $\left[\phi_{0}\right]$ is described by a stationary Gaussian random process.

The variance of the averaged travel-time or amplitude measurement is given by

$$
\sigma_{q}^{2}=\operatorname{Var}[\overline{\delta q}]=\frac{1}{N^{2}} \sum_{i=1}^{N} \sum_{j=1}^{N} \operatorname{Cov}\left[\delta q_{i}, \delta q_{j}\right] .
$$

The covariance between any two measurements $\left[\operatorname{Cov}\left[\delta q_{i}, \delta q_{j}\right]\right]$ depends on the reference cross-covariance function in the frequency domain,

$$
C_{0}(\omega)=\frac{1}{2 \pi} \int_{-T / 2}^{T / 2} C_{0}(t) e^{\mathrm{i} \omega t} \mathrm{~d} t,
$$




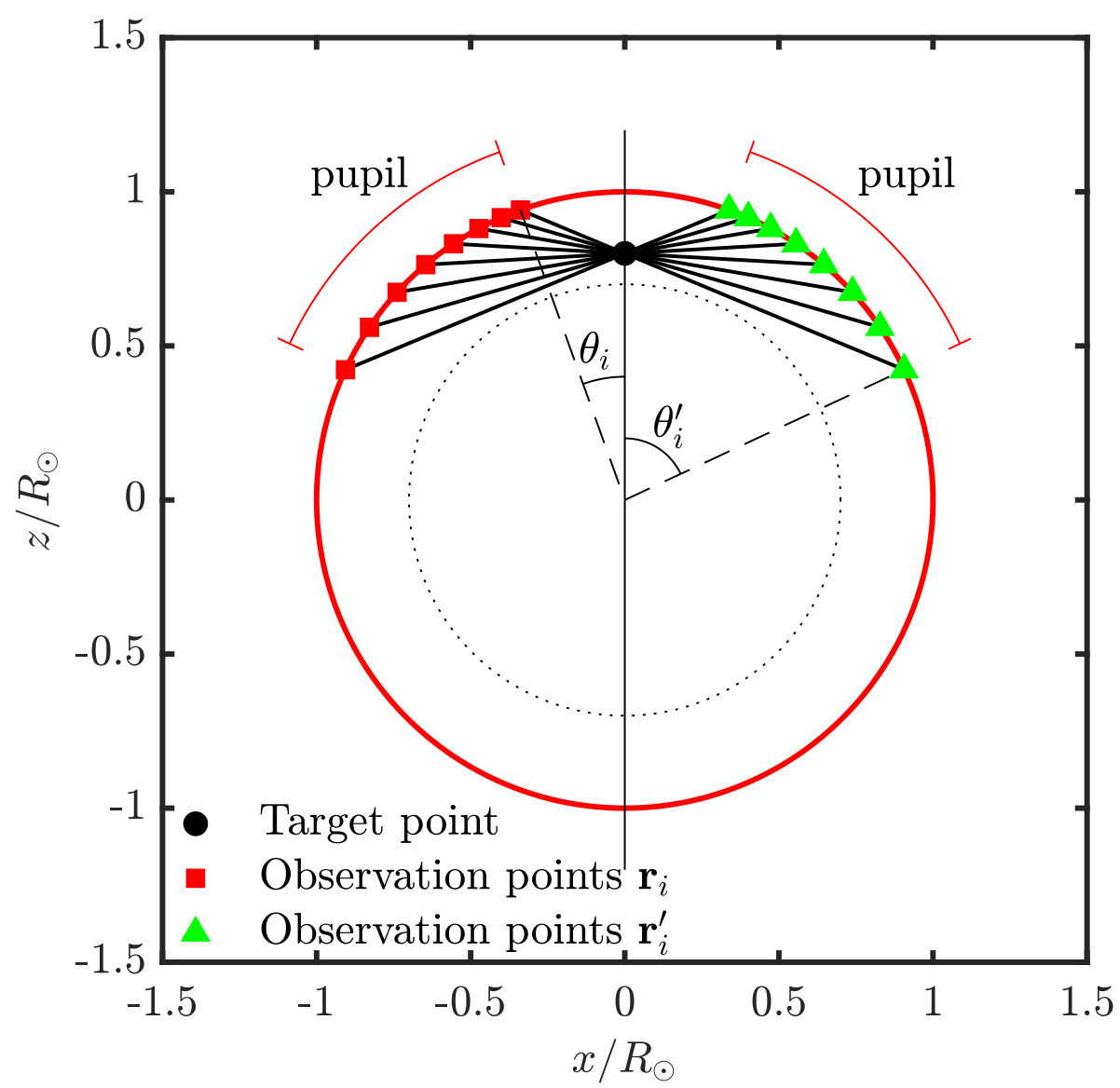

Figure 2.1: Sketch depicting the location of the observation points $\boldsymbol{r}_{i}$ (red squares) and $\boldsymbol{r}_{i}^{\prime}$ (green triangles) inside the pupil. The points are chosen such that the ray paths (black lines) intersect at a focus point at $z_{t}=0.8 \mathrm{R}_{\odot}$ (black dot). The dotted circle has a radius of $0.7 \mathrm{R}_{\odot}$.

and on the weighting functions $\left[W_{q}\right]$. Fournier et al. (2014) showed that the covariance is explicitly given by

$$
\begin{aligned}
\operatorname{Cov}\left[\delta q_{i}, \delta q_{j}\right]=\frac{(2 \pi)^{3}}{T} \int_{-\omega_{\max }}^{\omega_{\max }} \mathrm{d} \omega W_{q}^{*}\left(\boldsymbol{r}_{i}, \boldsymbol{r}_{i}^{\prime}, \omega\right)\left[W_{q}\left(\boldsymbol{r}_{j}, \boldsymbol{r}_{j}^{\prime}, \omega\right) C_{0}\left(\boldsymbol{r}_{i}^{\prime}, \boldsymbol{r}_{j}^{\prime}, \omega\right)\right. \\
\left.\quad \times C_{0}\left(\boldsymbol{r}_{i}, \boldsymbol{r}_{j}, \omega\right)+W_{q}^{*}\left(\boldsymbol{r}_{j}, \boldsymbol{r}_{j}^{\prime}, \omega\right) C_{0}\left(\boldsymbol{r}_{j}, \boldsymbol{r}_{i}^{\prime}, \omega\right) C_{0}\left(\boldsymbol{r}_{i}, \boldsymbol{r}_{j}^{\prime}, \omega\right)\right],
\end{aligned}
$$

where $\omega_{\max }=\pi / h_{t}$ is the Nyquist frequency and $h_{t}$ is the temporal cadence. The star denotes complex conjugation. Note that the noise covariance was originally derived for travel-time measurements, but it is easily extended to the amplitude measurements due to the linearity between $\delta a$ and $\delta C$. 


\subsection{Travel-time and amplitude sensitivity kernels for sound- speed perturbations to a uniform background medium}

\subsubsection{Wave equation and reference Green's function}

We consider the wave equation at angular frequency $\omega$,

$$
\mathcal{L} \phi(\boldsymbol{r}, \omega)=s(\boldsymbol{r}, \omega)
$$

where the wave operator is

$$
\mathcal{L}=\nabla^{2}+k^{2}(\boldsymbol{r}, \omega)
$$

and the wave number is given by

$$
k(\boldsymbol{r}, \omega)=\frac{\omega}{c(\boldsymbol{r})}(1+\mathrm{i} \gamma),
$$

where $c(\boldsymbol{r})$ is the sound speed and $\gamma$ is a constant number that accounts for attenuation. The random source of excitation $s(\boldsymbol{r}, \omega)$ is assumed to be stationary, uniformly distributed and spatially uncorrelated throughout the medium. Under these conditions, the expectation value of the cross-covariance function can be related directly to the imaginary part of the Green's function in the frequency domain (e.g. Gizon et al.2017)

$$
\langle C(\omega)\rangle=\frac{\Pi(\omega)}{\omega} \operatorname{Im} G\left(\boldsymbol{r}, \boldsymbol{r}^{\prime}, \omega\right),
$$

where the function $\Pi(\omega)$ is related to the frequency dependence of the source covariance. The angle brackets \langle\rangle represent the expectation value of a stochastic quantity.

We consider a background medium with the reference wave number

$$
k_{0}(\omega)=\frac{\omega}{c_{0}}(1+\mathrm{i} \gamma)
$$

where the reference sound speed is constant $c_{0}$. The reference Green's function is solution of $\mathcal{L}_{0} G_{0}\left(\boldsymbol{r}, \boldsymbol{r}^{\prime}\right)=\delta\left(\boldsymbol{r}-\boldsymbol{r}^{\prime}\right)$ where $\mathcal{L}_{0}=\nabla^{2}+k_{0}^{2}$ is the reference wave operator. Using a Sommerfeld radiation condition to avoid incoming waves at infinity, the expression for $G_{0}$ is

$$
G_{0}\left(\boldsymbol{r}, \boldsymbol{r}^{\prime}\right)=-\frac{e^{\mathrm{i} k_{0}\left\|\boldsymbol{r}-\boldsymbol{r}^{\prime}\right\|}}{4 \pi\left\|\boldsymbol{r}-\boldsymbol{r}^{\prime}\right\|}
$$

This simple analytic expression motivates the choice that we have made of a uniform medium.

\subsubsection{Perturbation to the cross-covariance function}

In this section we compute the perturbation to the cross-covariance $\left[\delta C=C-C_{0}\right]$ due to a small perturbation in sound speed $\left[\delta c(\boldsymbol{r})=c(\boldsymbol{r})-c_{0}\right]$. Using Equation 2.16, the expectation value of $\delta C$ is related to the perturbation to the Green's function,

$$
\langle\delta C(\omega)\rangle=\langle C(\omega)\rangle-C_{0}(\omega)=\frac{\Pi(\omega)}{\omega} \operatorname{Im}(\delta G) .
$$


Under the first-order Born approximation we have

$$
\mathcal{L}_{0} \delta G\left(\boldsymbol{r}, \boldsymbol{r}^{\prime}, \omega\right)=-\delta \mathcal{L} G_{0}\left(\boldsymbol{r}, \boldsymbol{r}^{\prime}, \omega\right),
$$

where $\delta \mathcal{L}=-2 k_{0}^{2} \delta c(\boldsymbol{r}) / c_{0}$ is the perturbation to the wave operator caused by the perturbations in the sound speed $[\delta c]$. According to Equation 2.20, the Born approximation is an equivalent-source description of wave interaction. Using $G_{0}$ to solve for $\delta G$, we find

$$
\delta G\left(\boldsymbol{r}_{i}, \boldsymbol{r}_{i}^{\prime}, \omega\right)=\int_{V} G_{0}\left(\boldsymbol{r}_{i}, \boldsymbol{r}, \omega\right) 2 k_{0}^{2} \frac{\delta c(\boldsymbol{r})}{c_{0}} G_{0}\left(\boldsymbol{r}, \boldsymbol{r}_{i}^{\prime}, \omega\right) \mathrm{d}^{3} \boldsymbol{r},
$$

where $V$ is the computational domain, including the full sphere. It follows that the perturbation to the cross-covariance between the points $\boldsymbol{r}_{i}$ and $\boldsymbol{r}_{i}^{\prime}$ is

$$
\left\langle\delta C_{i}(\omega)\right\rangle=\int_{V} \mathscr{C}_{i}(\boldsymbol{r}, \omega) \frac{\delta c(\boldsymbol{r})}{c_{0}} \mathrm{~d}^{3} \boldsymbol{r},
$$

where $\mathscr{C}_{i}(\boldsymbol{r}, \omega)$ is defined as

$$
\mathscr{C}_{i}(\boldsymbol{r}, \omega)=\frac{2 \Pi(\omega)}{\omega} \operatorname{Im}\left[k_{0}^{2} G_{0}\left(\boldsymbol{r}, \boldsymbol{r}_{i}, \omega\right) G_{0}\left(\boldsymbol{r}, \boldsymbol{r}_{i}^{\prime}, \omega\right)\right],
$$

where we used seismic reciprocity (the Green's function is unchanged upon exchanging source and receiver). Equation 2.23 shows that to compute the perturbation to the crosscovariance we need to compute a product of two Green's functions, one with a source at $\boldsymbol{r}_{i}$ and the other one with a source at $\boldsymbol{r}_{i}^{\prime}$.

\subsubsection{Travel-time and amplitude sensitivity kernels}

With the expression in hand for the perturbation to the cross-covariance, we now extract the travel-time and amplitude perturbations from the cross-covariance function. Using Equation 2.7 and Equation 2.22, the expectation value of the perturbation to the travel time $\left[\left\langle\delta \tau_{i}\right\rangle\right]$ and to the amplitude $\left[\left\langle\delta a_{i}\right\rangle\right]$ is given by

$$
\left\langle\delta q_{i}\right\rangle=2 \pi \int_{-\omega_{\max }}^{\omega_{\max }} W_{q}^{*}(\omega)\left\langle\delta C_{i}(\omega)\right\rangle \mathrm{d} \omega=\int_{V} K_{i}^{q}(\boldsymbol{r}) \frac{\delta c(\boldsymbol{r})}{c_{0}} \mathrm{~d}^{3} \boldsymbol{r}, \quad q \in\{\tau, a\},
$$

where $K^{q}$ are the point-to-point sensitivity kernels

$$
K_{i}^{q}(\boldsymbol{r})=2 \pi \int_{-\omega_{\max }}^{\omega_{\max }} W_{q}^{*}(\omega) \mathscr{C}_{i}(\boldsymbol{r}, \omega) \mathrm{d} \omega .
$$

Next we need to average the measurements for the deep-focusing technique as explained in Section 2.2.2. Using Equation 2.8, the expectation values of the averaged travel-time $\langle\overline{\delta \tau}\rangle$ and amplitude $\langle\overline{\delta a}\rangle$ perturbations can be written as

$$
\left\langle\overline{\delta q}\left(\boldsymbol{r}_{t}\right)\right\rangle=\frac{1}{N} \sum_{i=1}^{N}\left\langle\delta q_{i}\left(\boldsymbol{r}_{t}\right)\right\rangle=\int_{V} \overline{K^{q}}\left(\boldsymbol{r} ; \boldsymbol{r}_{t}\right) \frac{\delta c(\boldsymbol{r})}{c_{0}} \mathrm{~d}^{3} \boldsymbol{r}, \quad q \in\{\tau, a\},
$$

where $\overline{K^{q}}\left(\boldsymbol{r} ; \boldsymbol{r}_{t}\right)$ are the deep-focusing sensitivity kernels targeting a point at $\boldsymbol{r}_{t}$ defined by

$$
\overline{K^{q}}\left(\boldsymbol{r} ; \boldsymbol{r}_{t}\right)=\frac{1}{N} \sum_{i=1}^{N} K_{i}^{q}(\boldsymbol{r}), \quad q \in\{\tau, a\} .
$$




\subsection{Example calculations}

\subsubsection{Choice of numerical values and parameters}

In the following, the value of the reference sound speed is $c_{0}=10^{5} \mathrm{~m} \mathrm{~s}^{-1}$, the wave attenuation parameter is $\gamma=10^{-3}$, and $\mathrm{R}_{\odot}=696 \mathrm{Mm}$. The frequency dependence of the source covariance is chosen to be a Gaussian profile,

$$
\Pi(\omega)=\exp \left(-\frac{\left(|\omega|-\omega_{0}\right)^{2}}{2 \sigma^{2}}\right),
$$

where $\omega_{0} / 2 \pi=3 \mathrm{mHz}$ and $\sigma / 2 \pi=1 \mathrm{mHz}$. In our computations, we chose a temporal cadence $h_{t}=45 \mathrm{~s}$, i.e. the Solar Dynamics Observatory (SDO)/ Helioseismic and Magnetic Imager (HMI) cadence.

To compute the travel time and the amplitude, we have to define the window function $w$ in Equations 2.5-2.6. Since in this setup the cross-covariance function has a single branch, we chose a Heaviside step function:

$$
w(t)= \begin{cases}1 & \text { if } t>0 \\ 0 & \text { otherwise }\end{cases}
$$

Using the analytic expression for the Green's function (Equation 2.18) we obtain the reference cross-covariance $\left[C_{0}\right]$ (Equation 2.16). Figure 2.2 shows the travel-time and amplitude weighting functions $\left[W_{\tau}\right.$ and $W_{a}$ ] as a function of time for a pair of points separated by a distance of $D=1.2 \mathrm{R}_{\odot}$. The function $W_{a}$ is proportional to $C_{0}$ as stated by Equation 2.6, while $W_{\tau}$ is proportional to the temporal derivative of $C_{0}$ (Equation 2.5) and is thus shifted by one-fourth of a period.

\subsubsection{Point-to-point sensitivity kernels}

Using Equation 2.25, we compute the point-to-point travel-time and amplitude sensitivity kernels for sound-speed perturbations with a pair of points separated by $1.2 \mathrm{R}_{\odot}$. Crosssections through the point-to-point sensitivity kernels for the sound speed are shown in Figure 2.3. As already discussed in geophysics (Dahlen and Baig 2002) and in helioseismology (Gizon and Birch 2002), the travel-time kernel $\left[K^{\tau}\right]$ has small values along the geometrical ray path and the largest absolute values in the surrounding first Fresnel zone; see Figure 2.3 (a). The kernel changes sign multiple times away from the ray path when crossing higher-order Fresnel zones. On the other hand, the amplitude sensitivity kernel for sound-speed takes maximum absolute values along the ray path (Nolet et al. 2005), see Figure 2.3 (b). For a uniform background model, both point-to-point kernels are axially symmetric about the ray path. The total volume integrals of the two-point kernels are negative, $\int K^{\tau}(\boldsymbol{r}) \mathrm{d}^{3} \boldsymbol{r} \approx-8500 \mathrm{~s}$ and $\int K^{a}(\boldsymbol{r}) \mathrm{d}^{3} \boldsymbol{r} \approx-1.2$, which means that a uniform reduction in sound speed leads to a longer travel time and a larger cross-covariance amplitude.

\subsubsection{Deep-focusing sensitivity kernels}

With the point-to-point kernels for sound-speed perturbations in hand, we compute the deep-focusing sensitivity kernels for averaged travel time and amplitude using Equa- 


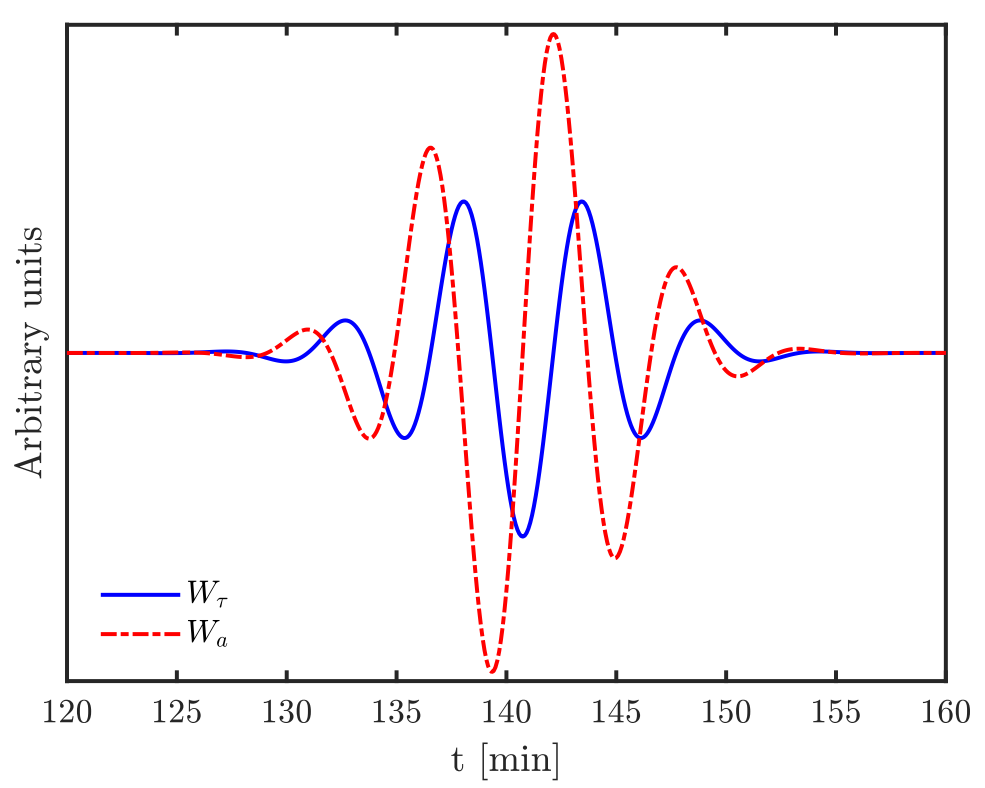

Figure 2.2: Sketch of the weighting functions for measuring travel times $\left[W_{\tau}\right]$ and crosscovariance amplitudes $\left[W_{a}\right]$. The function $W_{a}$ is proportional to the unperturbed crosscovariance function $\left[C_{0}\right]$. In this example, the two observation points are separated by a distance $D=1.2 \mathrm{R}_{\odot}$. The scalings for the functions $W_{\tau}$ and $W_{a}$ that are shown here are arbitrary as the two functions have different units.
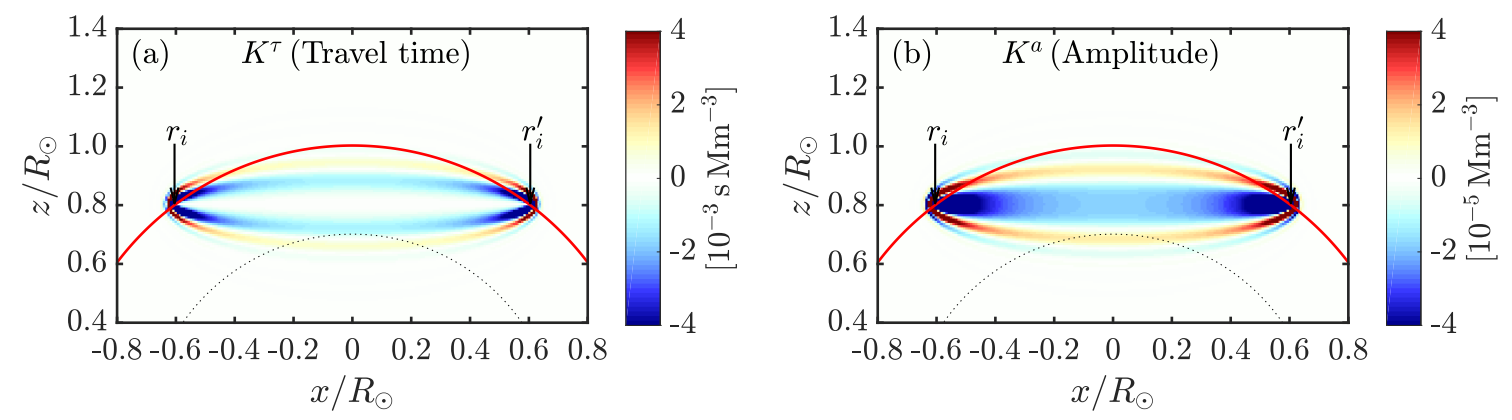

Figure 2.3: 2D cross-sections $(y=0)$ through the point-to-point kernels for sound-speed perturbations: (a) travel-time kernel $K^{\tau}(\boldsymbol{r})$ and (b) amplitude kernel $K^{a}(\boldsymbol{r})$. The pair of points $\left(\boldsymbol{r}_{i}, \boldsymbol{r}_{i}^{\prime}\right)$ on the surface are separated by $D=1.2 \mathrm{R}_{\odot}$. The dotted circle highlights radius $r=0.7 \mathrm{R}_{\odot}$.

tion 2.27. We consider all pairs of points in a pupil such that their ray paths intersect at a given target point along the $z$-axis. Neighboring observation points are separated in colatitude by a distance of approximately $\lambda_{\min } / 4 \approx 5 \mathrm{Mm}\left(0.41^{\circ}\right)$, where $\lambda_{\min }$ is the minimum wavelength used in this calculation. For a target point at radius $z_{t}=0.8 \mathrm{R}_{\odot}$, Figures 2.4(a) and 2.4(b) show 2D cross-sections $(y=0)$ through the deep-focusing sound-speed sensitivity kernels for $\overline{\delta \tau}$ and $\overline{\delta a}$. For travel-time measurements, the sensitivity is restricted to a shell surrounding the target location. In the case of amplitude measurements, the sensitivity is highly localized at the target point. This is a direct consequence of the structure 
2 Comparison of travel-time and amplitude measurements for deep-focusing time-distance helioseismology
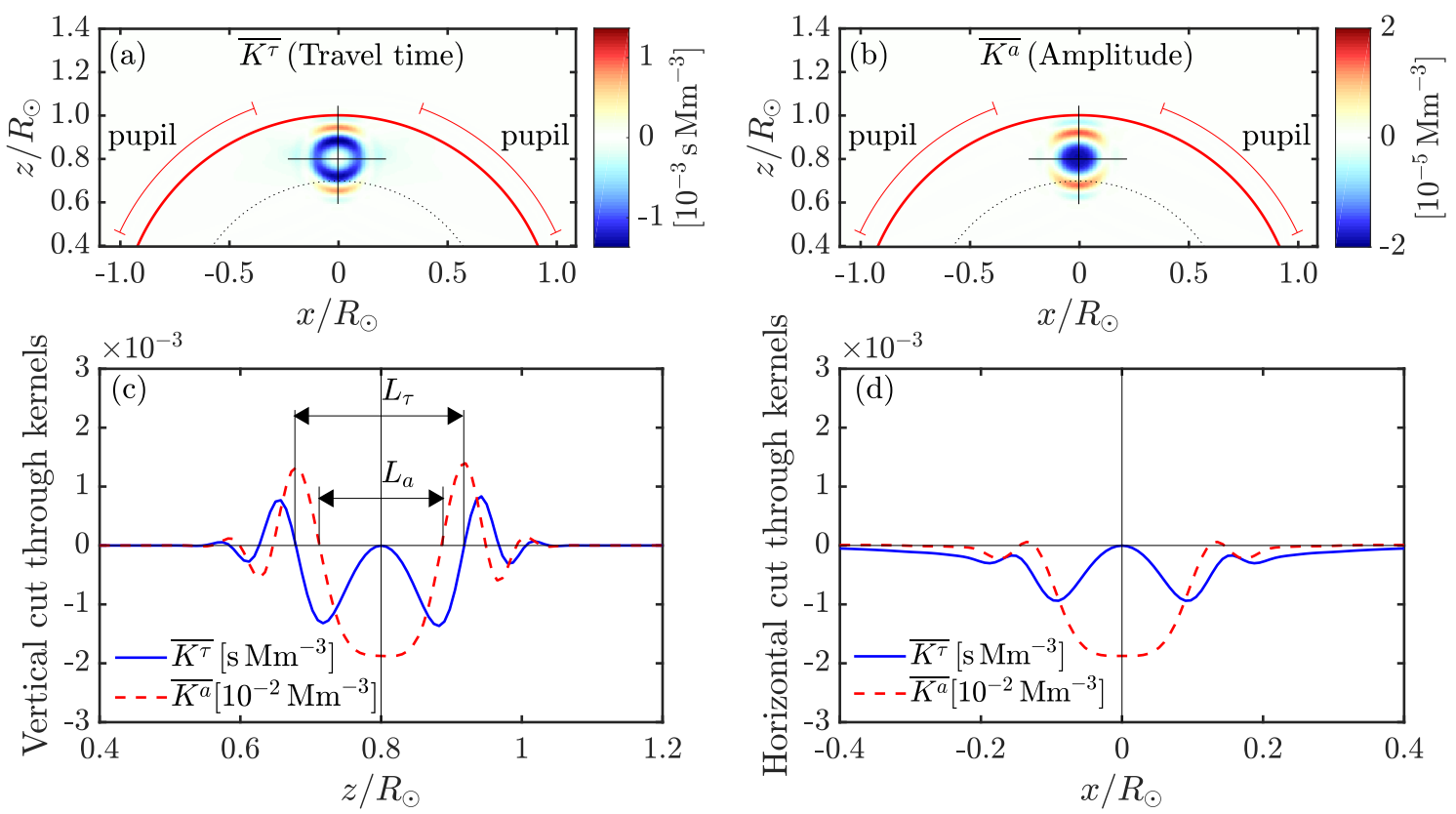

Figure 2.4: 2D cross-sections $(y=0)$ through the 3D deep-focusing $(\mathbf{a})$ sensitivity kernel for $\overline{\delta \tau}$ and (b) sensitivity kernel for $\overline{\delta a}$ averaged over the $N=N_{\theta} N_{\phi}$ observation points, where $N_{\theta}=158$ and $N_{\phi}=793$. The cross shows the target location, $x_{t}=y_{t}=0$, $z_{t}=0.8 \mathrm{R}_{\odot}$ which corresponds to the separation distance, $D=1.2 \mathrm{R}_{\odot}$ and the dots are situated at the surface at $z=0.7 \mathrm{R}_{\odot}$. (c) Vertical $(x=0, y=0)$ and (d) horizontal $\left(y=0, z=0.8 \mathrm{R}_{\odot}\right)$ cuts of the sensitivity kernels. $L_{\tau}$ and $L_{a}$ are the vertical widths of the deep-focusing kernels for travel time and amplitude, respectively.

of the point-to-point kernels depicted in Figure 2.3 . Figure 2.5 shows the same bottom panels as in Figure 2.4, but for a target point near the surface, $z_{t}=0.95 \mathrm{R}_{\odot}$. This target point leads to a shorter separation distance: $D=0.63 \mathrm{R}_{\odot}$.

\subsubsection{Kernel widths as functions of target depth}

The vertical widths of the deep-focusing sensitivity kernels for travel time $\left[L_{\tau}\right]$ and amplitude $\left[L_{a}\right]$ are defined in Figure 2.4 (c). This width indicates the extent of the central regions of a kernel, within which it keeps the same (negative) sign. The smaller $L_{\tau}$ (or $L_{a}$ ), the higher the spatial resolution of the travel-time (or amplitude) deep-focusing technique.

In Figure 2.6 the widths $L_{\tau}$ and $L_{a}$ are plotted as functions of the target radius $z_{t}$. The sensitivity kernels for amplitude measurements are better localized than those for traveltime measurements, at all depths, with $L_{a} \approx 0.7 L_{\tau}$. Furthermore $L_{\tau}$ and $L_{a}$ increase with target depth.

In order to better understand the data points, we consider a simplified version of the model presented in Section 2.3.2. For a single sound-speed scatterer (volume $\mathrm{d} V$ ) at position $\boldsymbol{r}$ in the mid-plane, the cross-covariance between observation points $\boldsymbol{r}_{i}$ and $\boldsymbol{r}_{i}^{\prime}$ can 

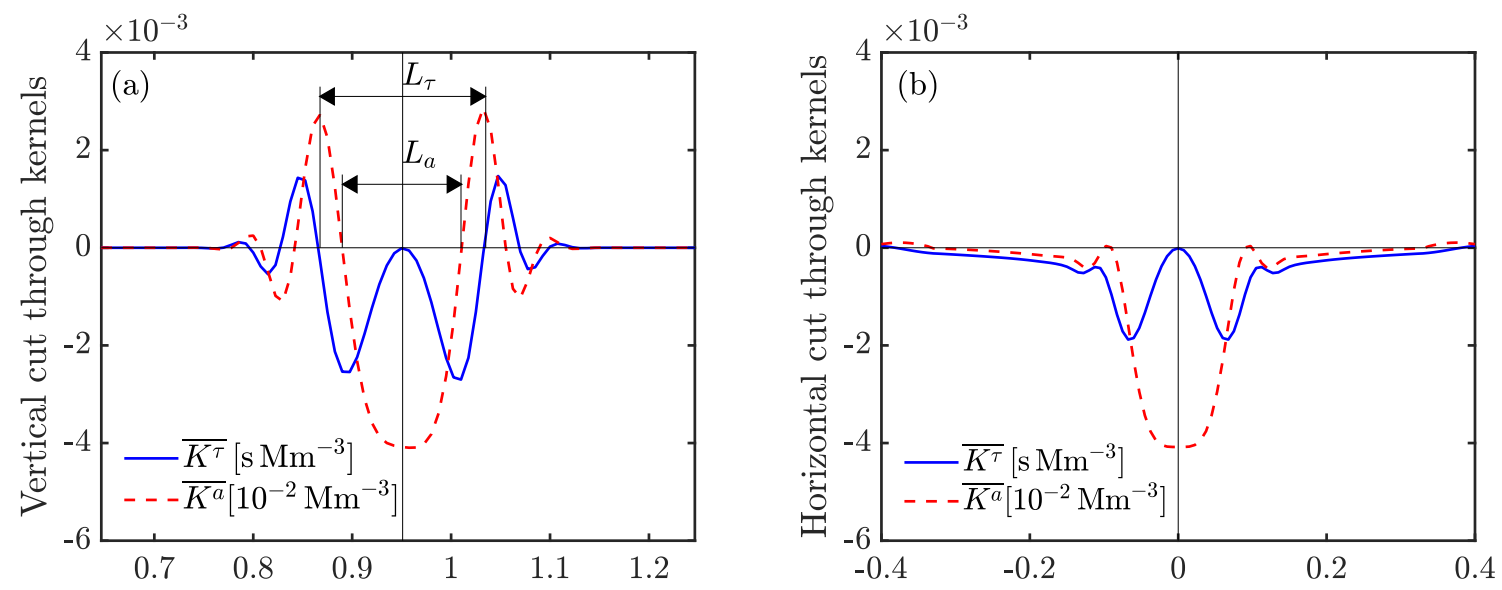

Figure 2.5: (a) Vertical ( $x=0, y=0$ ) and (b) horizontal $\left(y=0, z=0.95 \mathrm{R}_{\odot}\right)$ cuts of the sensitivity kernels for $\overline{\delta \tau}$ and $\overline{\delta a}$ for the target location, $x_{t}=y_{t}=0, z_{t}=0.95 \mathrm{R}_{\odot}$ which corresponds to the shorter separation distance $D=0.63 \mathrm{R}_{\odot}$.

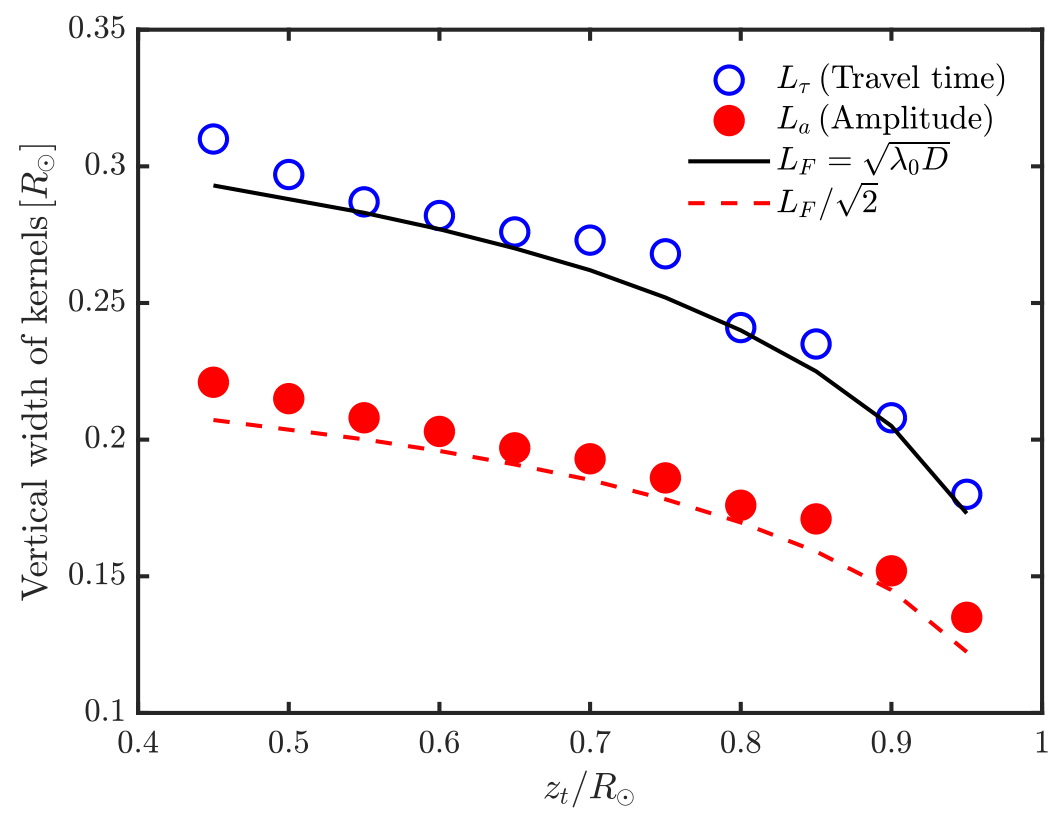

Figure 2.6: Vertical width of the deep-focusing sensitivity kernels for travel time $\left(L_{\tau}\right.$, open circles) and amplitude ( $L_{a}$, full circles) as a function of target position. The solid and dashed curves show the simple models described in Section 2.4.4. The width of the first Fresnel zone is approximately $L_{F}=\sqrt{\lambda_{0} D}$, where $D$ is the distance between the two points and $\lambda_{0}=33.3 \mathrm{Mm}$ is the wavelength at frequency $3 \mathrm{mHz}$.

be written

$$
\begin{array}{rlrl}
C\left(\boldsymbol{r}_{i}, \boldsymbol{r}_{i}^{\prime}\right) & \propto & \operatorname{Im}\left[G_{0}\left(\boldsymbol{r}_{i}^{\prime}, \boldsymbol{r}_{i}\right)+\tilde{\epsilon} G_{0}\left(\boldsymbol{r}_{i}^{\prime}, \boldsymbol{r}\right) G_{0}\left(\boldsymbol{r}, \boldsymbol{r}_{i}\right)\right], & \tilde{\epsilon}=2 k_{0}^{2} \frac{\delta c}{c_{0}} \mathrm{~d} V, \\
& \propto \operatorname{Im}\left[G_{0}\left(\boldsymbol{r}_{i}^{\prime}, \boldsymbol{r}_{i}\right)\left(1-\epsilon \mathrm{e}^{\mathrm{i} \Delta \phi}\right)\right], & \epsilon \approx \frac{\tilde{\epsilon}}{\pi D} .
\end{array}
$$


2 Comparison of travel-time and amplitude measurements for deep-focusing time-distance helioseismology

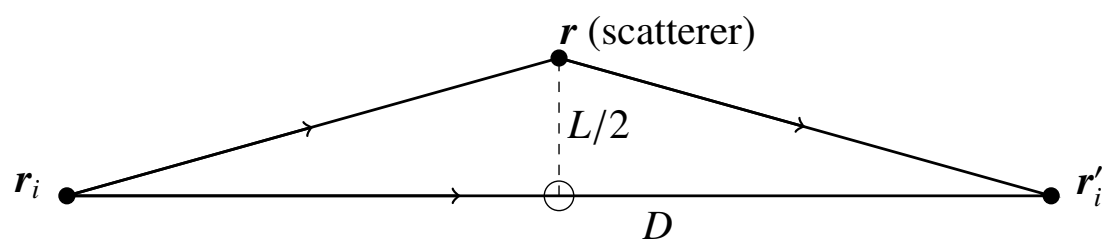

Figure 2.7: Sketch of the geometry discussed in Section 2.4.4. A scatterer is placed at equal distance to the two observation points. The scattered and direct wave fields combine at observation point $\boldsymbol{r}_{i}^{\prime}$. The open circle indicates the position of the target point.

In the above expression, $|\epsilon| \ll 1$ is the scattering amplitude. The phase perturbation $[\Delta \phi]$ is due to the difference $[\delta]$ between the path through the scatterer and the direct path between the two points $[D]$,

$$
\Delta \phi=2 \pi \frac{\delta}{\lambda_{0}}
$$

where $\lambda_{0}=33.3 \mathrm{Mm}$ is the reference wavelength. For a scatterer at equal distance from the two points (see Figure 2.7), we have

$$
\delta=2 \sqrt{(D / 2)^{2}+(L / 2)^{2}}-D \approx \frac{L^{2}}{2 D} .
$$

Thus the phase perturbation due to a scatterer midway between the two points and at a distance $L / 2$ from the direct path is approximately

$$
\Delta \phi \approx \frac{\pi L^{2}}{\lambda_{0} D}
$$

A travel time $[\tau]$ is most easily interpreted as a phase travel time. According to Equation 2.30, there is no phase change at the receiver when $\operatorname{Arg}\left(1-\epsilon \mathrm{e}^{\mathrm{i} \Delta \phi}\right)=0$, i.e. when $\Delta \phi=n \pi, n \in \mathbb{Z}$. In particular the sensitivity is zero along the direct ray path $(n=0)$. The width of the travel-time kernel coincides with the boundary of the first $(n=1)$ Fresnel zone:

$$
\Delta \phi_{\tau}=\pi \quad \text { and } \quad L_{\tau}=\sqrt{\lambda_{0} D} .
$$

This simple result was reported earlier in 2D by Gizon (2006).

Contrary to the travel-time perturbations, cross-covariance amplitude perturbations are extremal along the ray path (where $\Delta \phi=0$ ). The amplitude of the cross-covariance is unchanged when $\left|1-\epsilon \mathrm{e}^{\mathrm{i} \Delta \phi}\right|=1$. For small-amplitude perturbations, this condition is approximately $2 \epsilon \cos \Delta \phi=0$, i.e. $\Delta \phi=\pi / 2+m \pi$, with $m \in \mathbb{Z}$. Setting $m=0$ gives the width of the amplitude kernel:

$$
\Delta \phi_{a}=\pi / 2 \quad \text { and } \quad L_{a}=\sqrt{\lambda_{0} D / 2} .
$$

The dependence of the widths on target radius $z_{t}$ is understood through $D=4 \sqrt{R^{2}-z_{t}^{2}}$, so that $L \propto\left(R^{2}-z_{t}^{2}\right)^{1 / 4}$. As seen in Figure 2.6, the model values for $L_{\tau}$ and $L_{a}$ from Equations 2.34-2.35 are in reasonable agreement with the numerical values, including the relationship $L_{a}=L_{\tau} / \sqrt{2} \approx 0.7 L_{\tau}$. 


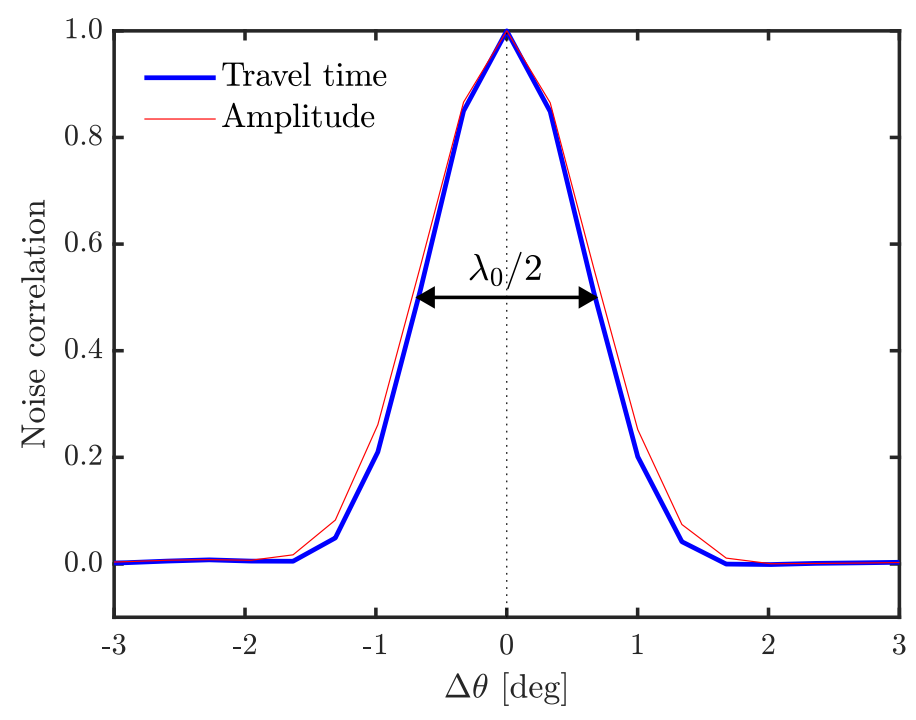

Figure 2.8: Cut through the noise correlation matrix for point-to-point travel times (solid curve) and point-to-point amplitude measurements (thin-red curve) as a function of the angular distance between neighboring points. The reference observation point is kept fixed at $\theta=45.2^{\circ}$ and the target radius is $z_{t}=0.8 \mathrm{R}_{\odot}$. The double-headed arrow indicates $\lambda_{0} / 2$, where $\lambda_{0}=33.3 \mathrm{Mm}$ is the wavelength at frequency $3 \mathrm{mHz}$.

\subsubsection{Noise covariance}

The model for the noise covariance matrix for travel-time and amplitude measurements was outlined in Section 2.2.3. Figure 2.8 shows a cut through the noise correlation matrix of point-to-point travel times and amplitudes. The correlation between neighboring pairs of points drops fast as a function of angular distance. For both travel-time and amplitude measurements, the correlation distance at half maximum is approximately $\lambda_{0} / 2$ (see also Gizon and Birch 2004). This justifies a posteriori why we chose points in the pupil that are separated by $\lambda_{0} / 4 \approx 8.3 \mathrm{Mm}$ to avoid under sampling.

\subsubsection{Localized sound-speed anomaly at $z_{0}=0.7 \mathrm{R}_{\odot}$}

In order to quantify the bias and variance of the travel-time and amplitude measurements in the present deep-focusing setup, we compute the travel-time and amplitude perturbations generated by sound-speed perturbations of our choosing (forward modeling).

In this section we consider a highly localized perturbation in sound speed with a Gaussian profile centered at $\boldsymbol{r}_{0}=\left(x_{0}, y_{0}, z_{0}\right)=\left(0,0,0.7 \mathrm{R}_{\odot}\right)$ such that

$$
\frac{\delta c(\boldsymbol{r})}{c_{0}}=-A_{1} \exp \left(-\left\|\boldsymbol{r}-\boldsymbol{r}_{0}\right\|^{2} / 2 s^{2}\right)
$$

where $A_{1}=0.02$. Notice that we have chosen a negative perturbation in sound speed. The parameter $s=0.03 \mathrm{R}_{\odot}$ determines the extent of the perturbation, which is roughly of the same size as the wavelength $\left(\lambda_{0} \approx 0.05 \mathrm{R}_{\odot}\right)$. The location of the perturbation is represented by the filled black circle in Figure 2.9(d). 
2 Comparison of travel-time and amplitude measurements for deep-focusing time-distance helioseismology
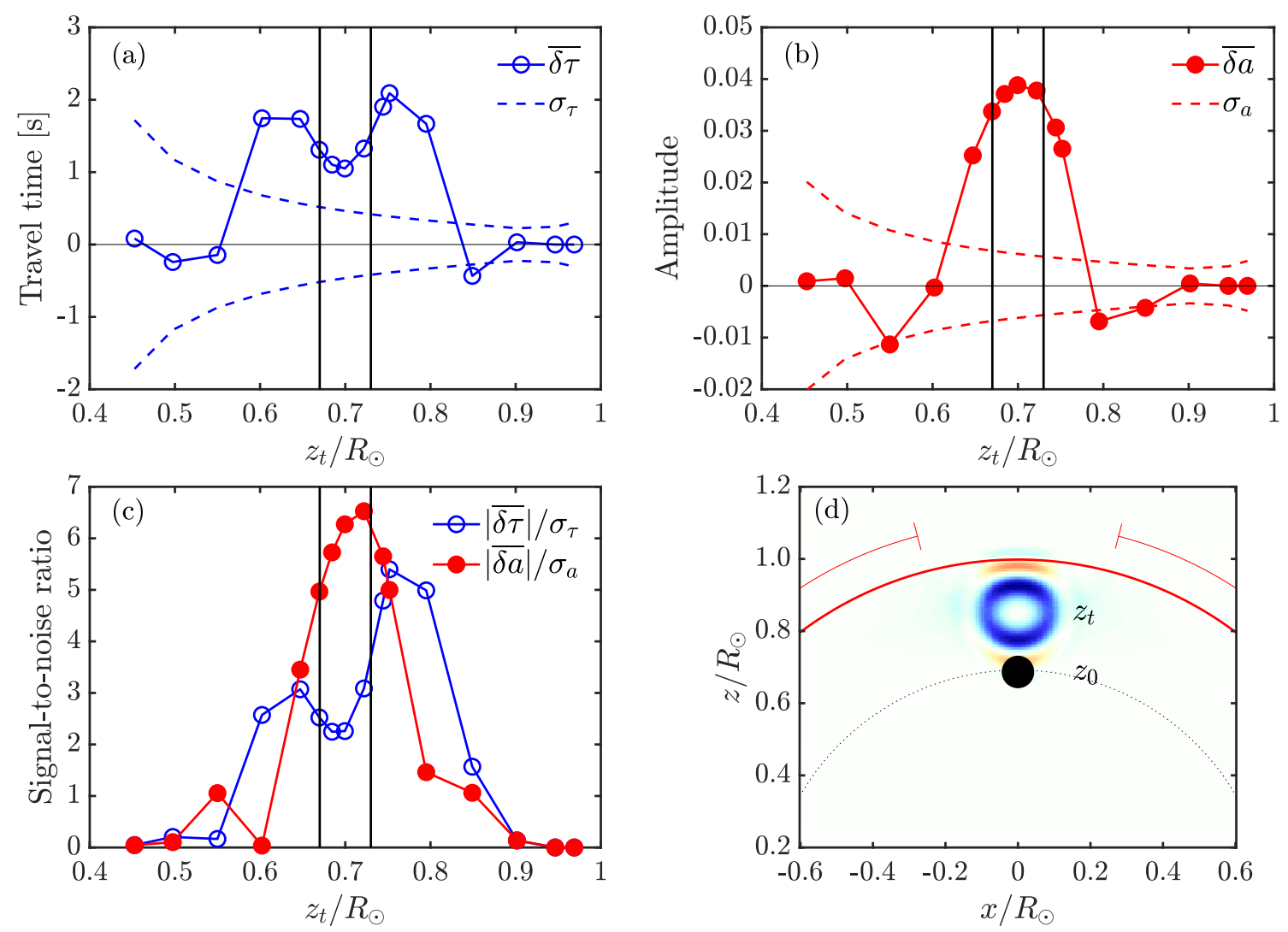

Figure 2.9: (a) Deep-focusing travel-time perturbation $\overline{\delta \tau}$ (connected open circles) and (b) amplitude perturbation $\overline{\delta a}$ (connected filled circles) due to $2 \%$ decrease in sound speed at radius $z_{0}=0.7 \mathrm{R}_{\odot}$ given by Equation 2.36. The dashed lines show the $1-\sigma$ level of stochastic noise for an observation duration $T=$ four years. The vertical lines indicate the width of the negative sound-speed perturbation $\left(z_{0} \pm s\right)$. (c) Signal-to-noise ratios for the deep-focusing travel-time and amplitude perturbations as functions of target radius. (d) Geometrical setup. The (negative) Gaussian perturbation in sound speed is indicated by the filled black circle at $\boldsymbol{r}_{0}=\left(x_{0}, y_{0}, z_{0}\right)=\left(0,0,0.7 \mathrm{R}_{\odot}\right)$. An example deep-focusing sensitivity kernel for $\overline{\delta \tau}$ is shown for the target radius $z_{t}=0.85 \mathrm{R}_{\odot}$.

Figure 2.9 (a) shows the deep-focusing travel-time measurements $[\overline{\delta \tau}]$ and the corresponding noise levels (standard deviations) for different target locations $\boldsymbol{r}_{t}=\left(0,0, z_{t}\right)$, where $0.4<z_{t} / \mathrm{R}_{\odot}<1$. Due to the hollow nature of the deep-focusing travel-time kernel, the signal is weaker at the depth where the perturbation is located than in the surroundings. The bulk of the perturbation is within $\left|z_{0}-z_{t}\right|<L_{\tau}\left(z_{0}\right) / 2 \approx 0.13 \mathrm{R}_{\odot}$. On the other hand, a maximum signal for the amplitude measurements is obtained at the radius where the perturbation is placed (Figure 2.9 (b)) due to the concentrated sensitivity of the deep-focusing kernel for amplitude measurements (Figure 2.4(b)).

To compare the two types of measurements, travel-time versus amplitude measurements, the signal-to-noise ratios are plotted in Figure 2.9(c). We find that the signal-tonoise ratio is higher and better localized for the amplitude measurements than for the travel-time measurements, given the highly localized perturbation in sound speed that we 

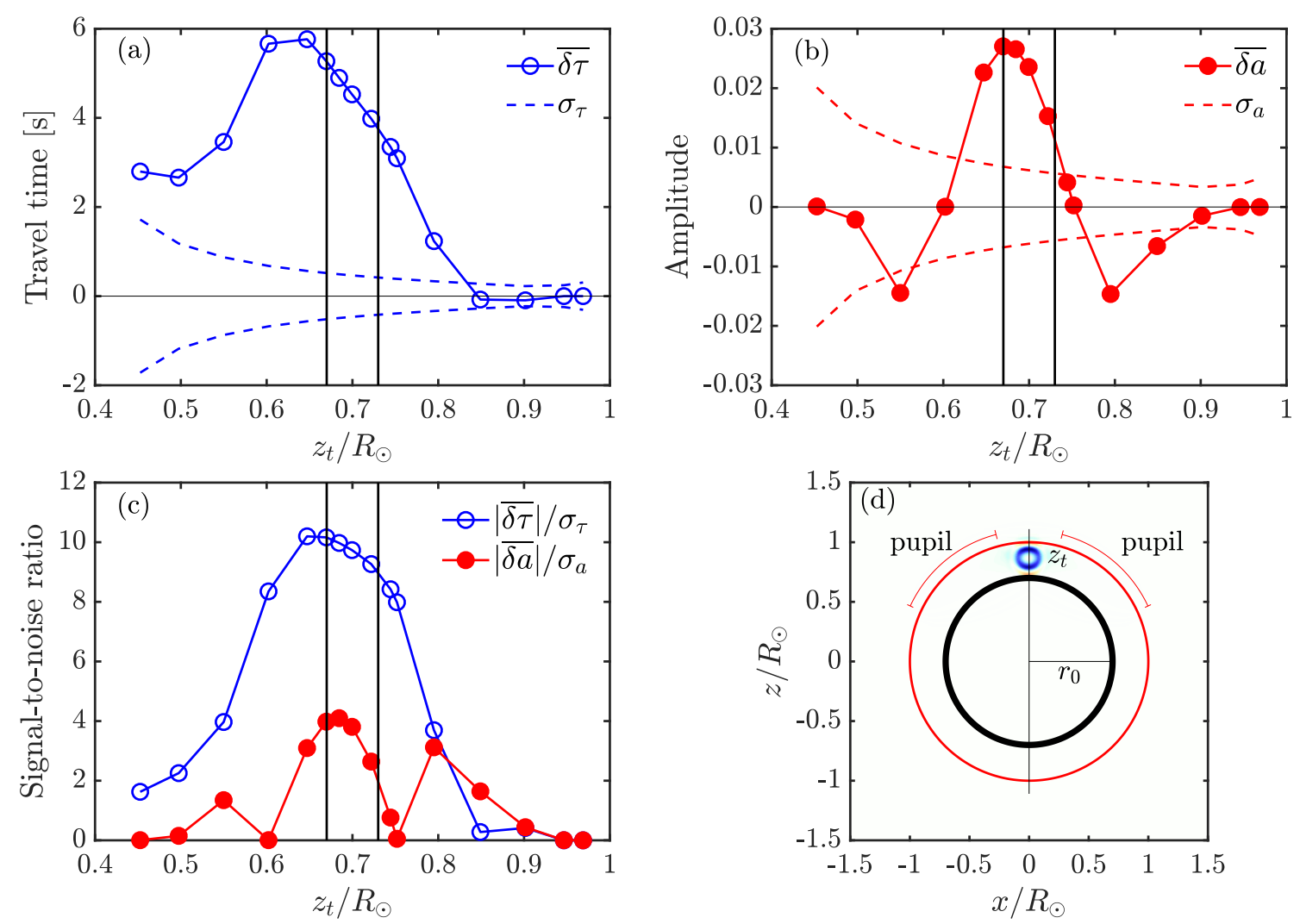

Figure 2.10: (a) Deep-focusing travel-time perturbation $\overline{\delta \tau}$ and (b) amplitude perturbation $\overline{\delta a}$ due to a $0.2 \%$ decrease in sound speed in a thin shell at radius $r_{0}=0.7 \mathrm{R}_{\odot}$ and defined by Equation 2.37. The vertical lines indicate the width of the negative soundspeed perturbation $\left(r_{0} \pm s\right)$. (c) Signal-to-noise ratios for the deep-focusing travel-time and amplitude perturbations versus target depth, for an observation duration $T=$ four years. (d) The thick black circle of thickness $2 s=0.06 \mathrm{R}_{\odot}$ indicates the location of the shell of sound-speed perturbation. The blue shades show a cut through a deep-focusing travel-time sensitivity kernel with target radius $z_{t}=0.85 \mathrm{R}_{\odot}$.

chose in this section.

\subsubsection{Sound-speed anomaly in a shell at radius $r_{0}=0.7 \mathrm{R}_{\odot}$}

The search for solar-cycle changes at the bottom of the solar convection zone is a key question in helioseismology. In this section we consider a shell of perturbation in sound speed at radius $r_{0}=0.7 \mathrm{R}_{\odot}$ with a profile given by

$$
\frac{\delta c(\boldsymbol{r})}{c_{0}}=-A_{2} \exp \left(-\left(\|\boldsymbol{r}\|-r_{0}\right)^{2} / 2 s^{2}\right)
$$

where $A_{2}=0.002$ and $s=0.03 \mathrm{R}_{\odot}$. This shell of negative sound-speed perturbation is illustrated in Figure 2.10(d). As in the previous section the radial extent of this perturbation is of the order of a wavelength. 
The corresponding travel-time and amplitude perturbations, as well as the noise levels for $T=$ four years, are shown in Figures 2.10 (a) and 2.10 (b). We see that the travel-time and amplitude signals peak below $z_{t}=0.7 \mathrm{R}_{\odot}$ : the deep-focusing averaging scheme is not unbiased. For a shell-like perturbation, the signal-to-noise ratio is twice as large for the travel-time measurements as for the amplitude measurements (Figure 2.10(c)).

\subsection{Conclusion}

In this article we considered toy models in a uniform background medium to study the localization and noise properties of the deep-focusing time-distance technique. We considered two measurement quantities extracted from the cross-covariance function: travel times and amplitudes. The sensitivity kernels for sound speed were derived under the first Born approximation.

We computed the spatial sensitivity of travel-time and amplitude to perturbations in sound speed with respect to a uniform background medium. We find that the travel-time sensitivity to sound-speed perturbations is zero at the target location and negative in a surrounding region with diameter $L_{\tau} \approx\left(\lambda_{0} D\right)^{1 / 2}$, where $\lambda_{0}$ is the wavelength and $D$ is the travel distance between the points used in the deep-focusing averaging. On the other hand, the amplitude sensitivity peaks at the target location and is negative in a region with diameter $L_{a} \approx\left(\lambda_{0} D / 2\right)^{1 / 2}$, resulting in a higher signal-to-noise ratio for small-scale perturbations. We conclude that amplitude measurements are a useful complement to travel-time measurements in local helioseismology.

In future studies, we intend to extend this work to a standard solar model using accurate computations of Green's functions in the frequency domain. We also intend to study the capability of the deep-focusing technique to recover flows in the solar interior. Deep-focusing travel times have already been used to recover meridional circulation (e.g. Rajaguru and Antia 2015). No significant improvement is expected from using deep-focusing amplitude measurements to recover such slowly varying flows. However, amplitude measurements should help resolve flows that vary on scales smaller than the wavelength, e.g. convective flows.

\section{Acknowledgements}

We thank Thomas L. Duvall, Aaron C. Birch, Zhi-Chao Liang, Chris S. Hanson, and Kaori Nagashima for useful discussions and comments. MP is a member of the International Max Planck Research School (IMPRS) for Solar System Science at the University of Göttingen. The computer infrastructure was provided by the German Data Center for SDO funded by the German Aerospace Center (DLR) and by the Ministry of Science of the State of Lower Saxony, Germany. 


\title{
3 Single-cell meridional flow in the solar convection zone ${ }^{1}$
}

\begin{abstract}
The Sun's meridional flow is the axisymmetric poloidal component of motion. It has been hypothesised to transport toroidal magnetic flux towards the equator near the bottom of the convection zone, producing the sunspot butterfly diagram. However, a debate rages as to the structure of the flow inferred from helioseismology. Here we determine the meridional flow over the last two sunspot cycles using two data sets (SOHO/MDI and GONG). Confidence in the results is provided by their agreement during the overlap period 20012011. The flow in each sunspot cycle is equatorward at the base of the convection zone with an amplitude of about $4 \mathrm{~m} / \mathrm{s}$ at latitude $45^{\circ}$. This is consistent with flux-transport dynamo models, provided the toroidal magnetic flux is stored below 0.8 solar radius, where the flow is measured to be equatorward.
\end{abstract}

\subsection{Report}

The rotation of the Sun interacts with convection to drive global-scale axisymmetric flows (Ruediger 1989). These are the Sun's differential rotation (the poles rotate once every 34 days, the equator every 25 days) and meridional flow. The differential rotation (Thompson et al. 1996) plays an essential role in the solar dynamo: it winds up poloidal magnetic flux to create toroidal magnetic fields. The role of the meridional flow at the surface is to transport poloidal magnetic flux towards the poles. The role of the subsurface meridional flow is less certain - it might or might not play a crucial role. In the class of models known as flux-transport dynamos, it plays the critical role of advecting the subsurface toroidal field equatorwards, thus explaining the observed equatorward drift of the butterfly wings and setting the period of the dynamo (Wang et al. 1991, Choudhuri et al. 1995). The flux-transport dynamo model makes testable predictions in this regard. Firstly, the flow should be equatorward near the base of the convection zone and, secondly, it should have the correct amplitude to reproduce the observed drift of the butterfly wings.

\footnotetext{
${ }^{1}$ This chapter reproduces the submitted version of the article Meridional flow in the Sun's convection zone is a single cell in each hemisphere by L. Gizon, R. H. Cameron, M. Pourabdian, Z.-C. Liang, D. Fournier, A. C. Birch, and C. S. Hanson, published in Science, 368 (6498), 1469-1472 (2020). Contribution statement: MP developed the inversion setup and inverted the travel times in collaboration with DF. MP also contributed to the interpretation of the results and to the writing of the article.
} 
Testing these predictions has proved to be difficult. From the theoretical side the meridional flow is difficult to compute from first principles, as it results from a small imbalance between two large terms (Kitchatinov 2016). Both one-cell and two-cell meridional flow geometries have been advocated by theory and numerical simulations (Kitchatinov 2016, Pipin and Kosovichev 2018, Featherstone and Miesch 2015). The basic problem from the theoretical side lies in the difficulty of correctly modeling the effect of rotation on the turbulent convection. From the observational side the question has been tackled with helioseismology, i.e. the use of solar acoustic oscillations to infer flows and other perturbations in the solar interior. Giles et al. (1997) demonstrated the capability of time-distance helioseismology to detect the meridional flow throughout the convection zone using SOHO/MDI space observations for the month of June 1996. Later studies inferred the meridional flow over different time periods and from different data sets; see Böning (2017) for a survey of observational results. Using the more recent SDO/HMI space observations starting from 2010, several authors reported conflicting geometries for the meridional flow: one or two cells in the radial direction (Zhao et al. 2013, Jackiewicz et al. 2015, Rajaguru and Antia 2015, Chen and Zhao 2017, Mandal et al. 2018). Liang et al. (2018) joined the MDI data with the HMI data (overlap of only one year in 2010) and found an inferred flow structure that is different between the two data sets. These differences may result from different instrumental systematic errors, the calibration of the observations, and different assumptions in the data analysis.

To establish whether a helioseismic inference is trustworthy, it is essential to compare the answers from two independent data sets, covering an extended overlap period. Indeed, the comparison between the data from MDI and from the ground stations operated by the Global Oscillation Network Group (GONG, Harvey et al. (1996)) was key in validating the global mode inversions for subsurface differential rotation and their temporal variations (Howe 2009).

In this paper we study the structure and time variability of the meridional flow at the base of the convection zone, using the latest helioseismic techniques. The raw observations for this study are Dopplergrams at reduced spatial resolution, which provide information about acoustic modes with spherical harmonic degrees up to 300 . We restrict ourselves to reduced resolution data because they are known to be less prone to instrumental errors (Giles 2000). We considered the three main data sets: MDI, HMI, and GONG. The MDI medium-degree program consists of Dopplergrams with $192 \times 192$ pixels per frame for the period May 1996 to April 2011 (Kosovichev et al. 1997). After April 2003, the SOHO spacecraft was rotated by $180^{\circ}$ every three months: we only use the observations when MDI was right-side up to ensure consistency of the data (Liang and Chou 2015). The HMI data we use here cover the period from May 2010 to April 2019. They were processed by the HMI Team to have a format $(204 \times 204$ pixels $)$ similar to the MDI medium-degree data (Scherrer et al. 2012). The third dataset is constructed from the merged fully calibrated GONG++ Dopplergrams for August 2001 to April 2019 $(839 \times 839$ pixels $)($ Harvey et al. 1998$)$ by applying Gaussian smoothing and downsampling to $200 \times 200$ pixels.

The MDI, HMI, and GONG images are remapped onto the same heliographic coordinate system (scale of $0.6^{\circ}$ per pixel) and tracked at the Carrington rotation rate $(456.03 \mathrm{nHz})$. In doing so, the MDI data are corrected to account for a misalignment of the instrument with respect to the spacecraft corresponding to a $0.20^{\circ}$ error in the solar $P$ angle. As de- 
scribed by Liang et al. (2017), this was determined using HMI images as reference during the 2010 overlap period. The orientation of HMI images is known to better than $0.01^{\circ}$ thanks to the Venus transit from 5-6 June 2012 (Couvidat et al.2016). In addition, MDI, HMI and GONG images are all corrected for a $0.08^{\circ}$ error in the inclination of the solar rotation axis to the ecliptic with respect to the traditional value of $7.25^{\circ}$ measured by $\mathrm{R}$. C. Carrington in 1863 (Section 3.2).

The above data are analyzed using time-distance helioseismology (Duvall et al.|1993). Solar subsurface flows leave their signature in the time it takes for acoustic waves to travel between two points on the surface, A and B. The Doppler velocity signals at A and B are cross-correlated to measure the travel times both from A to B and in the opposite direction. The difference between the two travel times is mostly sensitive to the flows near the geometrical ray path that connects $\mathrm{A}$ and $\mathrm{B}$ through the interior. To learn about the meridional flow, we consider points separated in latitude, using the quadrant geometry described in detail by Liang et al. (2018). In particular, the angular width of the arc geometry is $30^{\circ}$. The points in locations of strong magnetic fields are excluded from the quadrant averages (Liang and Chou 2015). We measure the south minus north travel-time difference, $\tau(\Delta, \lambda)$, where $\Delta$ is the angular distance between the quadrants and $\lambda$ is the latitude of the mid point. Using a one-parameter fit (Gizon and Birch 2004) to the crosscorrelation functions computed daily, we measure the travels times for angular distances in the range from $6^{\circ}$ to $42^{\circ}$ and latitudes within $\pm 54^{\circ}$. The south-north travel times are corrected for the center-to-limb effect determined from the travel-time differences in the east-west direction (Zhao et al.2012), see Figure 3.5.

Figures 3.1A to 3.1 C show the measured travel times averaged over three ranges of travel distances at low latitudes in the north and south. To compare the travel times from the different instruments, we included in the averages shown in this figure only those days when travel times are available for two instruments: GONG and MDI (right side up) during 2001-2011, and GONG and HMI after 2010. The travel times have been binned down to a sampling of 2.9 years to aid in the comparison of the three data sets. The signs of the measured travel times are consistent with a poleward meridional flow near the surface. The time variations are related to the sunspot number (Figure 3.1D) as a result of the surface inflows into active regions (Gizon 2004). The most important finding from Figures 3.1A to 3.1 C (and Figure 3.6) is that the MDI and GONG observations are in good agreement during their overlap period from 2001 to 2011 (Section 3.2). The agreement is at a level of $0.1 \mathrm{~s}$, a number which is much smaller than the $1 \sigma$ error bars which reflect the realization noise due to the random excitation of the (same) acoustic waves. In contrast, the HMI travel times are offset with respect to the GONG travel times, with very small values in the northern hemisphere. From this point on, we set aside the HMI travel times as we were unable to find the source of this inconsistency. For the period from May 1996 to May 2001, we also considered data from the early GONG network with lower-resolution cameras. The corresponding travel times are consistent with the MDI data for the same period, however they are more noisy (Section 3.2). All of the above considerations lead us to combine the MDI data from May 1996 to April 2003 and the GONG data thereafter.

The travel times are linearly related to the meridional flow via three-dimensional travel-time sensitivity kernels, computed in the first Born approximation to account for finite wavelength effects (Fournier et al. 2018). These kernels are constructed to be con- 

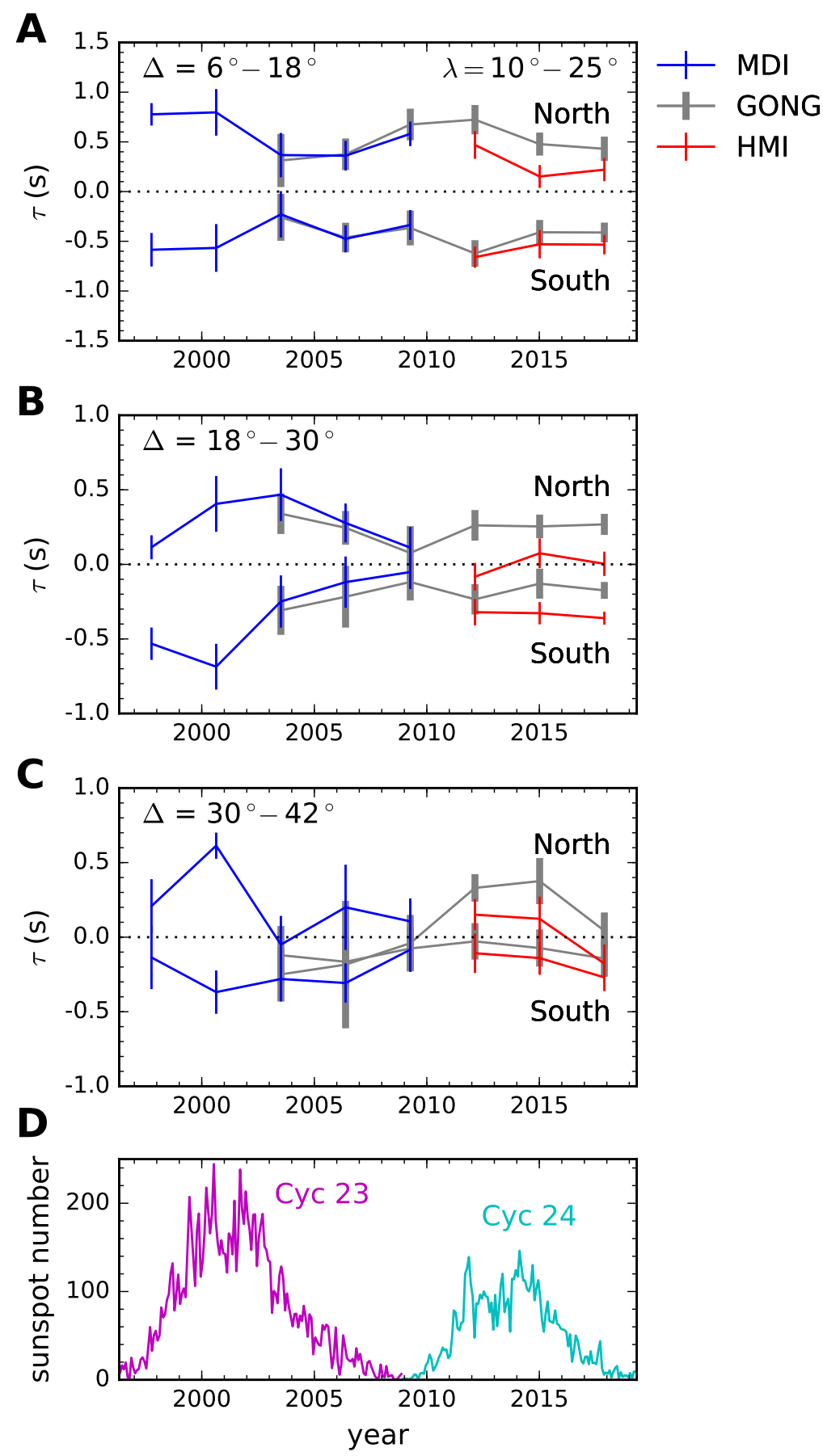

Figure 3.1: Averages of the helioseismic travel times. The observations are split into eight segments of 2.9 years and are averaged over latitudes $\lambda=10^{\circ}$ to $25^{\circ}$ in each hemisphere. For the three segments between 2002 and 2010, only the days when travel times for both MDI (right side up) and GONG are available are included in the averages. For the three segments from 2010, only the days when travel times for both HMI and GONG are available are included in the averages. The three top panels show these travel times averaged over angular distances $\Delta$ in the range $(\mathbf{A})$ from $6^{\circ}$ to $18^{\circ},(\mathbf{B})$ from $18^{\circ}$ to $30^{\circ}$, and $(\mathbf{C})$ from $30^{\circ}$ to $42^{\circ}$. (D) Monthly sunspot number versus time, displaying sunspot cycles 23 and 24 . 
sistent with the travel-time measurement procedure (a one-parameter fit). They rely on the accurate computation of Green's functions using a finite-element solver in the frequency domain (Gizon et al. 2017). In order to formulate the inverse problem in matrix form, we represent the radial and colatitudinal components of the meridional flow, $U_{r}$ and $U_{\theta}$, as linear combinations of cubic B-splines in the radial direction in the convection zone and Legendre polynomials in the theta direction. The base of the convection zone is fixed at radius $r_{b}=0.713 R_{\odot}$ determined by global helioseismology (Christensen-Dalsgaard et al. 1991). Symbolically, the full set of travel times (vector $\tau$, no averaging over $\lambda$ or $\Delta$ ) is related to the flow coefficients (vector $u$ ) via the linear equation $\tau=K u+n$, where $K$ is a matrix of kernel coefficients and $n$ is the noise vector. The noise is specified through a model for the noise covariance matrix (Fournier et al.2014), scaled on the diagonal using the observations. With all these ingredients at hand we can solve the inverse problem under the physical constraints that the flow is mass conserving and that it does not cross the convection zone boundaries. A trade-off between noise and bias exists and is chosen via a regularization parameter $\alpha$. Linear inversions were validated using synthetic data (Section 3.2). We made two choices for the regularization parameter. With the first choice, $\alpha=\alpha_{11}$, it is possible to unambiguously distinguish between one- and two-cell flow profiles with 11 years of data, with a noise level of about $1.5 \mathrm{~m} / \mathrm{s}$ at the base of the convection zone. The second choice, $\alpha=\alpha_{3}$, is applied to data segments of three years; it is such that the bias is maintained while the noise increases to $2.5 \mathrm{~m} / \mathrm{s}$. These small errors are consistent with previous estimates. The noise level for inferences of a meridional flow covering the bottom quarter of the convection zone was estimated by Braun and Birch (2008) to be about $1 \mathrm{~m} / \mathrm{s}$ for twelve years of observations, without accounting for mass conservation. Woodard (2009) found very similar error estimates based on mode-coupling analysis.

Figure 3.2A shows the inferred $U_{\theta}$ in the convection zone, averaged over each cycle. The first seven years uses MDI data, the remainder the GONG data. For each cycle, the flow takes the form of a single cell in each hemisphere: poleward at the surface (Figure $3.3 \mathrm{C}$ ) and equatorward at the base of the convection zone (Figure 3.3 A). This result does not depend in which of the four seasons the observations were made (Section 3.2). The flow switches sign near 0.79 solar radius (Figure $3.3 \mathrm{~B}$ ), which is consistent with previous inversions that employed the constraint of mass conservation (Rajaguru and Antia 2015, Mandal et al. 2018). The latitudinal flow profile at the base of the convection zone is well approximated by $U_{b} \sin 2 \theta$ with $U_{b}=4.8 \pm 1.0 \mathrm{~m} / \mathrm{s}$ for cycle 23 and $U_{b}=3.6 \pm 1.0 \mathrm{~m} / \mathrm{s}$ for cycle 24 (Figure $3.3 \mathrm{~A}$ ). As seen from the stream functions in Figure $3.2 \mathrm{~B}$, the boundary between the two cells lies near the equator. To further investigate the time variations, we show the three-year averages in Figures $3.2 \mathrm{C}$ and $3.2 \mathrm{D}$ and Figures $3.3 \mathrm{D}$ and $3.3 \mathrm{E}$. The noise is higher than for the eleven-year averages, as expected. Except for the period near minimum of activity between the two cycles, we see a single cell in each hemisphere. At the surface, the variations of $U_{\theta}$ at $30^{\circ}$ latitude are significant and anti-correlated with the sunspot number (Figure $3.3 \mathrm{E}$ ). We note that in the middle of the convection zone (see Figure 3.8), the meridional flow averaged over cycle 24 is poleward in the north and very weak in the south. Looking at the three-year averages, there is an apparent decrease of the amplitude of $U_{\theta}$ from about 2004 during the decaying phase of cycle 23 . This decrease is seen in both MDI and GONG data.

The agreement between MDI (right side up) and GONG is better for angular distances $\Delta \leq 30^{\circ}$ than for $\Delta=30^{\circ}-42^{\circ}$ (see Figure 3.1). As shown by Liang et al. (2018) using a 

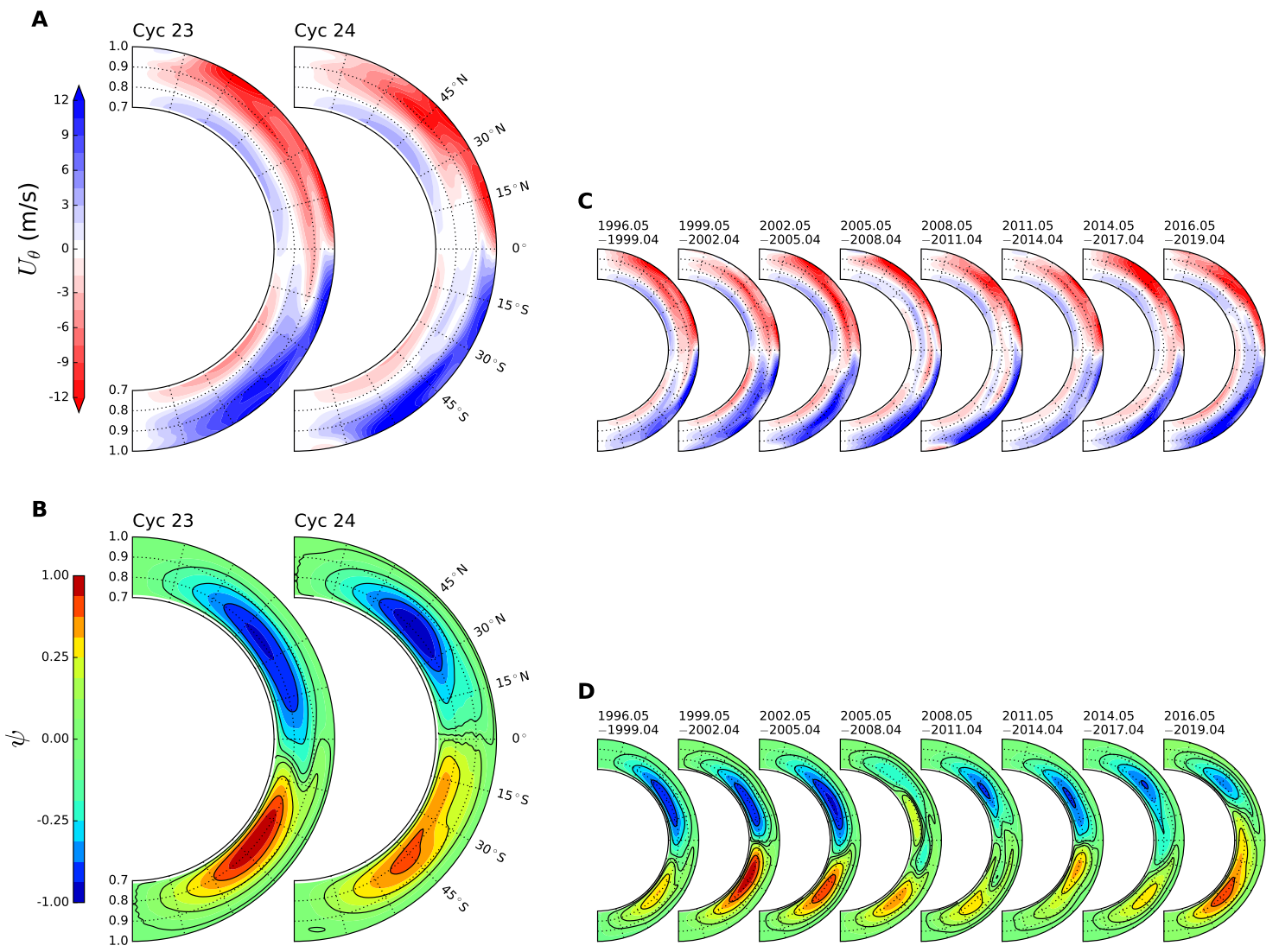

Figure 3.2: Inferred meridional flow in the solar convection zone. The first seven years consist of MDI data, the next 16 years of GONG data. (A) Flow $U_{\theta}$ for each sunspot cycle (for $U_{r}$ see Figure 3.7). (B) Stream function $\psi$ for each sunspot cycle, defined by $\rho \boldsymbol{U}=\nabla \times[\hat{\boldsymbol{\phi}} \psi /(r \sin \theta)]$, where $\rho$ is the density and $\hat{\boldsymbol{\phi}}$ is the unit vector in the longitudinal direction. The stream function is normalized to its maximum absolute value. Panels $\mathbf{C}$ and $\mathbf{D}$ show the temporal variations using three-year segments.

ray theory approach, travel distances less than $30^{\circ}$ are capable of distinguishing between single- and double-cell models. Thus, we also performed inversions for $\Delta \leq 30^{\circ}$ only. The results, given in Figures 3.9 to 3.12 , confirm the single-cell solution for each cycle for the MDI and GONG data. In addition, the inversions for MDI and GONG restricted to the days in common and separation distances $\Delta \leq 30^{\circ}$ are almost indistinguishable (Figure 3.13).

Figure $3.4 \mathrm{~A}$ shows the surface $U_{\theta}$ smoothed in time with a low pass filter (5 years) and its relation to the sunspot butterfly pattern. The time variations of the flow occur near the bands of activity, are largely antisymmetric across the equator, and can be understood as local inflows around active regions. These inflows are likely driven by the horizontal pressure gradients caused by the surface magnetic field (Spruit 2003, Gizon and Rempel 2008). They are possibly related to the time variations of the zonal flows (Vorontsov et al. 2002). The smoothed $U_{\theta}$ at the base of the convection zone has much smaller and less significant time variations (Figure 3.4B). As said above, the basic idea of fluxtransport dynamo models is that the toroidal flux is transported by $U_{\theta}$ at the base of the convection zone to explain the butterfly diagram at the surface. To test this idea, we 

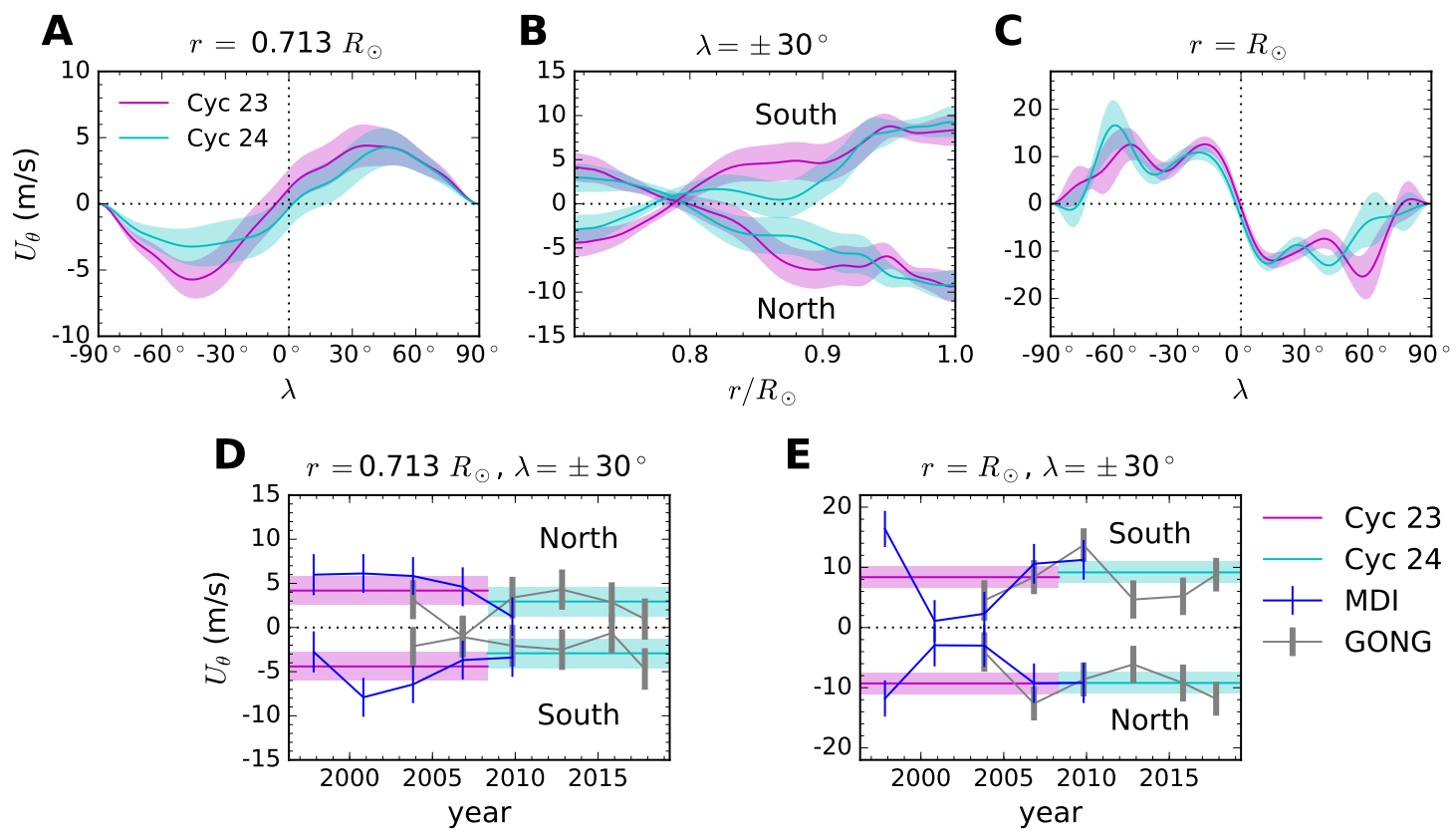

Figure 3.3: Cuts through the solutions shown in Figure 3.2A and 3.2 C. (A) Latitudinal dependence of $U_{\theta}$ at the base of the convection zone for cycles 23 and 24. (B) Radial dependence of $U_{\theta}$ at $\pm 30^{\circ}$ latitude, for each cycle. The flow switches sign near $r=0.79 R_{\odot}$. (C) Latitudinal dependence of $U_{\theta}$ at the surface, for each cycle. (D) Time dependence at $\pm 30^{\circ}$ latitude at the base of the convection zone. Both the cycle averages (Cyc 23 and Cyc 24) and the three-year averages are shown. (E) Time dependence of $U_{\theta}$ at $\pm 30^{\circ}$ latitude at the surface.

used $U_{\theta}$ from each cycle averaged from the bottom of the convection zone to $0.8 R_{\odot}$ to drive a 1-D mean-field equation governing the evolution of the longitudinal component of the magnetic field (Section 3.2). The observed surface radial magnetic field obtained from the KP/NSO, SOLIS and HMI telescopes was used. To compare the model with the observations, Figure 3.4 $\mathrm{C}$ shows the observed toroidal flux at the surface obtained from WSO observations, which corresponds to the rate of flux emergence through the surface (Cameron et al. 2018). The latitude of the peak subsurface toroidal field shows a clear equatorial propagation consistent with the equatorward drift of the emergence band.

The inferred one-cell meridional flow in each hemisphere is able to explain the equatorial migration of the sunspots under a simple flux-transport model. Neither latitudinal turbulent pumping nor dynamo-wave behaviour is required as the dominant cause of the equatorial migration of sunspots.

\section{Acknowledgements}

We dedicate this paper to the memory of Michael J. Thompson. This work is a tenyear team effort by the Solar and Stellar Interiors Department at MPS. We thank Raymond Burston for help with the netDRMS data management system. We thank Hélène Barucq and the Magique-3D team at Inria Bordeaux Sud-Ouest and UPPA for making 


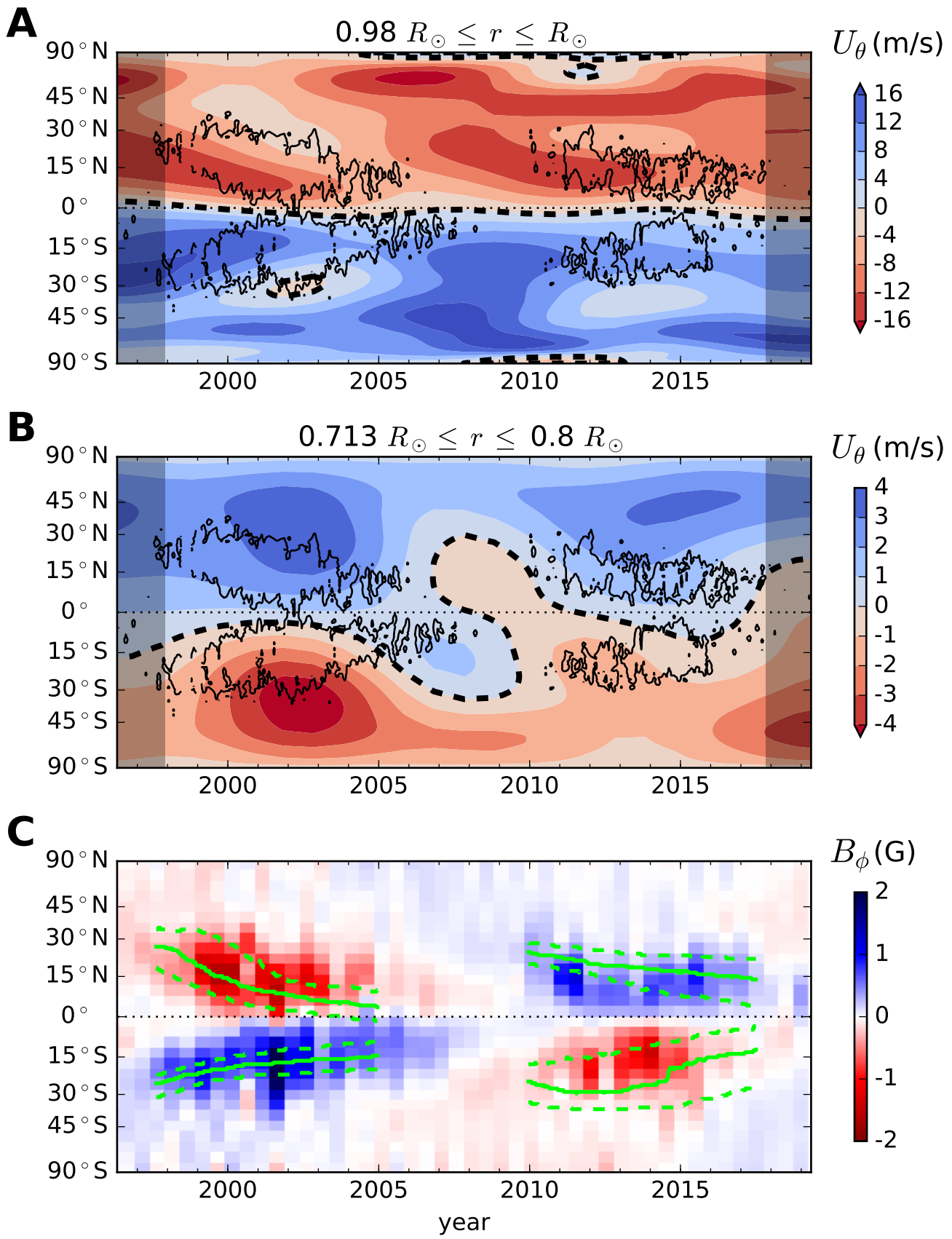

Figure 3.4: Solar butterfly diagram in relation to the inferred meridional flow. (A) Contours of line-of-sight magnetic field at the surface and $U_{\theta}$ averaged over the top $2 \%$. The flow inversion uses all travel distances (MDI first 7 years, GONG next 16 years) and variations on time scales faster than 5 years are filtered out. The inflows into active region latitudes dominate the time dependence of $U_{\theta}$. The dashed line shows where $U_{\theta}=0$. (B) Same contours of the magnetic field at the surface and $U_{\theta}$ averaged over $r_{b} \leq r \leq 0.8 R_{\odot}$ (dashed line where $U_{\theta}=0$ ). (C) Toroidal field from WSO observations (Cameron et al. 2018) and, superimposed, latitudes at which the toroidal field from the flux transport model, based on the measured $U_{\theta}$, is maximum. The solid green curves and the error bars (dashed green) are the median and the 16 and 84 percentiles, obtained from 300 realizations of the flow. 
available the FEM wave solver Montjoie. SOHO is a project of international cooperation between ESA and NASA. This work utilizes GONG data obtained by the NSO Integrated Synoptic Program (NISP), managed by the National Solar Observatory, the Association of Universities for Research in Astronomy (AURA), Inc. under a cooperative agreement with the National Science Foundation. The HMI data used are courtesy of NASA/SDO and the HMI science team. The sunspot numbers are from WDC-SILSO, Royal Observatory of Belgium, Brussels. Funding: The data were processed at the German Data Center for SDO, funded by the German Aerospace Center under grant DLR 50 OL 1701. We acknowledge partial support from ERC Synergy grant WHOLE SUN 810218. The Center for Space Science at NYU Abu Dhabi is funded by the NYUAD Institute under grant G1502. M.P. is a member of the International Max Planck Research School (IMPRS) for Solar System Science at the University of Göttingen. Author contributions: L.G. and Z.-C.L. designed the research. Z.-C.L. measured the travel times. D.F. and C.H. computed the sensitivity kernels for flows. M.P. and D.F. inverted the travel times. R.H.C. developed the flux-transport dynamo model. L.G. and R.H.C. wrote the first draft of the paper. All authors discussed the results and contributed to the final version of the paper. Competing interests: The authors declare no competing interests. Data and materials availability: The MDI data are from the JSOC export tool at http://jsoc.stanford.edu/ajax/lookdata.html?ds=mdi.vw_v for the period May 1996-April 2011. The HMI data are from the JSOC export tool at http://jsoc.stanford.edu/ajax/lookdata.html?ds=hmi.vw_v_45s for the period May 2010-April 2019. The GONG merged velocity data mrvzi are from https://g ong2.nso.edu/ archive/patch.pl?menutype=g for GONG date 960501 to 190501. The measured travel times and the sensitivity kernels may be obtained from the authors upon request.

\subsection{Supplementary materials: Materials and methods}

\subsubsection{Helioseismic travel times}

Correction of Carrington element. We note that Kholikov and Hill (2014) measured the travel times from the GONG data. They reported a strong seasonal dependence of the travel times connected to the $B_{0}$ angle. For the small travel distances, this seasonal dependence can be largely attributed to a known $0.08^{\circ}$ error in the Carrington element describing the inclination of the Sun's rotation axis to the ecliptic (see, e.g., Fig. 2 of Hathaway and Rightmire (2010)). We corrected for this effect in our study, following Liang et al. (2019) (Appendix A.1 of Liang et al. (2019)).

Consistency between MDI and GONG++ travel times. Here we compare the MDI (right side up) and GONG++ travel times shown in Figure 3.1 for the three time segments between 2002 and 2010, which use only the days when travel times are available for both instruments. We denote the MDI travel times by $\tau_{i}$ and the GONG++ travel times by $\tau_{i}^{\prime}$, where $i=1,2,3$ labels the time segments for particular ranges in latitude and distance. The corresponding standard deviations are $\sigma_{i}$ and $\sigma_{i}^{\prime}$. For each $i$, the random errors in measurements $\tau_{i}$ and $\tau_{i}^{\prime}$ are strongly correlated because the noise is associated with the 
same realization of acoustic sources inside the Sun. The correlation coefficient between $\tau_{i}$ and $\tau_{i}^{\prime}$, denoted by $\rho_{i}$, is measured to be approximately 0.9 .

To check if the two datasets are consistent, we construct the paired difference

$$
d=\frac{1}{N} \sum_{i=1}^{N}\left(\tau_{i}-\tau_{i}^{\prime}\right)
$$

where $N=3$ is the number of independent time segments. In the absence of bias (the null hypothesis), the variance of $d$ is

$$
\sigma^{2}=\frac{1}{N^{2}} \sum_{i=1}^{N}\left(\sigma_{i}^{2}+{\sigma^{\prime}}_{i}^{2}-2 \rho_{i} \sigma_{i} \sigma_{i}^{\prime}\right) .
$$

The ratio $d / \sigma$ indicates the level at which the null-hypothesis that $\tau_{i}$ and $\tau_{i}^{\prime}$ have the same mean can be rejected. Table 3.1 gives these quantities for different distance and latitude ranges. We see that the MDI and GONG++ travel times agree at the $2 \sigma$ level. In particular, for the most important distance range of $18^{\circ}$ to $30^{\circ}$, the travel times have a mean difference $d \simeq 0.07 \mathrm{~s}$, which is a very tight constraint on the systematic differences (see also section on the comparison of inversions of the two datasets). The exception is the distance range $30^{\circ}$ to $42^{\circ}$ in the northern hemisphere where the null hypothesis can be rejected at the $3 \sigma$ level, however these distances do not contribute strongly to the inversions (see Section 3.2.5 'Tests with synthetics').

\begin{tabular}{cccrcr}
\hline \hline Figure & & $\Delta$ & $d$ & $\sigma$ & $d / \sigma$ \\
\hline $3.1 \mathrm{~A}$ & North & {$\left[6^{\circ}, 18^{\circ}\right]$} & $-0.018 \mathrm{~s}$ & $0.043 \mathrm{~s}$ & -0.4 \\
& South & {$\left[6^{\circ}, 18^{\circ}\right]$} & $0.015 \mathrm{~s}$ & $0.046 \mathrm{~s}$ & 0.3 \\
$3.1 \mathrm{~B}$ & North & {$\left[18^{\circ}, 30^{\circ}\right]$} & $0.065 \mathrm{~s}$ & $0.037 \mathrm{~s}$ & 1.8 \\
& South & {$\left[18^{\circ}, 30^{\circ}\right]$} & $0.075 \mathrm{~s}$ & $0.042 \mathrm{~s}$ & 1.8 \\
$3.1 \mathrm{C}$ & North & {$\left[30^{\circ}, 42^{\circ}\right]$} & $0.244 \mathrm{~s}$ & $0.067 \mathrm{~s}$ & 3.6 \\
& South & {$\left[30^{\circ}, 42^{\circ}\right]$} & $-0.103 \mathrm{~s}$ & $0.069 \mathrm{~s}$ & -1.5 \\
\hline
\end{tabular}

Table 3.1: Consistency test between MDI and GONG++ travel times, averaged as in Figure 3.1 A to $\mathrm{C}$, for individual hemispheres and distance ranges.

Early GONG data. Since we use medium-resolution Dopplergrams in this paper, we also considered the early GONG data, also called GONG-Classic data, available from 7 May 1995 until 13 June 2001 (Hughes et al. 2016). In Figure 3.14, we show a version of Figure 3.1 including the GONG-Classic travel times from 1 May 1996 to 31 May 2001. As can be seen, the agreement for the days in common with MDI (right-side up) is reasonable. However the noise in the GONG-Classic data is somewhat higher than MDI by a factor of 1.5 to 2, because GONG-Classic has less acoustic power at larger spherical harmonic degrees. Thus we keep the combination of MDI (right-side up) and GONG++ for the Cycle-23 averages. 


\subsubsection{Discretization of the meridional flow}

To parametrize the meridional flow we introduce the basis functions

$$
\phi_{j}(r, \theta)=Q_{k}(r) P_{l}(\cos \theta), \quad j:=(k, l),
$$

where the $Q_{k}$ are cubic B-splines in the radial direction (with 18 knots uniformly-spaced from $r_{b}=0.713 R_{\odot}$ to $R_{\odot}$ ) and the $P_{l}$ are Legendre polynomials of degrees up to $l=15$ in the horizontal direction. The meridional flow is written as a linear combination of these basis functions,

$$
\begin{aligned}
U_{\theta}(r, \theta) & =\sum_{j=1}^{M} v_{j} \phi_{j}(r, \theta), \\
U_{r}(r, \theta) & =\sum_{j=1}^{M} w_{j} \phi_{j}(r, \theta),
\end{aligned}
$$

where the $w_{j}$ and $v_{j}$ are the flow coefficients to be determined. Each flow component is described by $M=N_{r} N_{h}$ coefficients, where $N_{r}=18+2=20$ and $N_{h}=16$ are the number of basis functions in the radial and horizontal directions.

\subsubsection{Forward problem}

The forward problem of time-distance helioseismology relates a flow model (described by the flow coefficients $w_{j}$ and $v_{j}$ ) to the travel times through sensitivity kernels. These three-dimensional kernels are obtained using the first Born approximation to account for finite-wavelength effects (Gizon et al.2017). They depend on the accurate computation of Green's functions. For this, we use the finite-element solver Montjoie in the frequency domain (Chabassier and Durufle 2016) and a realistic background solar model (ChristensenDalsgaard et al. 1996). Since the meridional flow is axisymmetric, the kernels are averaged over longitude (Fournier et al. 2018) to obtain the two-dimensional kernels $\mathcal{K}^{r}$ and $\mathcal{K}^{\theta}$, such that

$$
\tau_{i}=\int_{0}^{\pi} \int_{r_{b}}^{R_{\odot}}\left(\mathcal{K}_{i}^{r} U_{r}+\mathcal{K}_{i}^{\theta} U_{\theta}\right) \mathrm{d} r \mathrm{~d} \theta,
$$

where the index $i$ corresponds to a particular combination of $(\lambda, \Delta)$. Only frequencies in the range $2-5 \mathrm{mHz}$ are kept to account for the details of the measurement procedure. Example kernels are shown in Figure 3.15.

The travel times $\tau_{i}$ have noise $n_{i}$ due to the stochastic nature of solar oscillations. The noise is specified by the $N \times N$ covariance matrix

$$
\Lambda_{i j}=\mathbb{E}\left[n_{i} n_{j}\right],
$$

where $\mathbb{E}$ denotes the expectation value. It is computed under the assumption of stationary and spatially homogeneous sources of wave excitation (Fournier et al. 2014).

Combining equations (3.4), (3.5) and (3.6), we recast the problem in matrix form:

$$
\tau=K u+n, \quad u=\left[\begin{array}{l}
w \\
v
\end{array}\right],
$$


where $\tau=\left[\tau_{1} \cdots \tau_{N}\right]^{T}$ is the vector of travel times, $u$ is the vector of flow coefficients of length $2 M$, and $n$ is the noise vector. The matrix $K=\left[K^{r} K^{\theta}\right]$ is a matrix of kernel coefficients of size $N \times 2 M$, whose elements are obtained by projecting the above kernels onto the basis functions:

$$
K_{i j}^{\beta}=\int_{0}^{\pi} \int_{r_{b}}^{R_{\odot}} \mathcal{K}_{i}^{\beta} \phi_{j} \mathrm{~d} r \mathrm{~d} \theta,
$$

where $\beta$ refers to either $r$ or $\theta$.

\subsubsection{Inverse problem}

To invert for the travel times, we use the regularized least squares method (Tarantola 2005) and seek flow coefficients $u$ that minimize

$$
\left\|\Lambda^{-1 / 2}(K u-\tau)\right\|^{2}+\alpha\|D u\|^{2}
$$

where $\|\cdot\|$ is the discrete $L^{2}$ norm, $D$ is a regularization matrix, and $\alpha$ is a regularization parameter. We choose $D u$ to be the discretization of the weighted flow vorticity $\left(\partial_{r}\left(r U_{\theta}\right)-\right.$ $\left.10 \partial_{\theta} U_{r}\right) / r$, which accounts for the fact that $U_{r}$ is much smaller than $U_{\theta}$ throughout most of the convection zone.

The minimization of the cost function is subject to the linear constrains $C u=0$ (mass conservation) and $S u=0$ (flow confined to the convection zone). These constraints are implemented via Lagrange multipliers vectors $\kappa$ and $\mu$ to be determined.

Projecting the equation for mass conservation $\operatorname{div} \rho \boldsymbol{U}=0$ onto the basis functions and integrating by parts, we obtain the constraint in matrix form:

$$
C u=0,
$$

where $C=\left[C^{r} C^{\theta}\right]$ is an $M \times 2 M$ matrix with elements

$$
C_{i j}^{\beta}=\int_{0}^{\pi} \int_{r_{b}}^{R_{\odot}} \phi_{i} \partial_{\beta} \phi_{j} \rho r^{2} \sin \theta \mathrm{d} r \mathrm{~d} \theta
$$

and $\partial_{\beta}$ stands for either the $r$ or $\theta$ component of the gradient in polar coordinates.

The matrix $S$ imposes that the flow does not cross the convection zone boundaries,

$$
S u=0 .
$$

It consists of four sub-matrices, one for each section of the contour:

$$
S=\left[\begin{array}{cc}
S^{1} & 0 \\
S^{2} & 0 \\
0 & S^{3} \\
0 & S^{4}
\end{array}\right] \quad \begin{aligned}
& \left(r=r_{b}\right) \\
& \left(r=R_{\odot}\right) \\
& (\theta=\pi)
\end{aligned}
$$

with $S^{1}$ and $S^{2}$ obtained by projecting the flow $U_{r}$ at the bottom and top of the convection zone onto Legendre polynomials, and $S^{3}$ and $S^{4}$ are obtained by projecting the flow $U_{\theta}$ on the rotation axis onto the B-splines:

$$
\begin{aligned}
S_{l^{\prime},(k, l)}^{1} & =\delta_{l^{\prime} l} Q_{k}\left(r_{b}\right) \\
S_{l^{\prime},(k, l)}^{2} & =\delta_{l^{\prime} l} Q_{k}\left(R_{\odot}\right) \\
S_{k^{\prime},(k, l)}^{3} & =\delta_{k^{\prime} k} P_{l}(1) \\
S_{k^{\prime},(k, l)}^{4} & =\delta_{k^{\prime} k} P_{l}(-1) .
\end{aligned}
$$




\begin{tabular}{cc|cc}
\hline \hline \multicolumn{2}{c|}{ single-cell } & \multicolumn{2}{c}{ double-cell } \\
$r_{i} / R_{\odot}$ & $F_{i}$ & $r_{i} / R_{\odot}$ & $F_{i}$ \\
\hline 1.000 & -15 & 1.000 & -15 \\
0.880 & -7 & 0.980 & -14 \\
0.800 & 0 & 0.915 & 0 \\
0.713 & 4.877942 & 0.850 & 10 \\
& & 0.800 & 0 \\
& & 0.713 & -2.361903 \\
\hline
\end{tabular}

Table 3.2: Coordinates of points used to construct the radial profiles of the two synthetic flow models

The matrix $S$ has size $\left(2 N_{r}+2 N_{h}\right) \times 2 M$.

The saddle point of the cost function with respect to the flow coefficients $u$ and the Lagrange multipliers $\kappa$ and $\mu$ leads to the matrix equation

$$
\left[\begin{array}{ccc}
K^{T} \Lambda^{-1} K+\alpha D^{T} D & C^{T} & S^{T} \\
C & 0 & 0 \\
S & 0 & 0
\end{array}\right]\left[\begin{array}{l}
u \\
\kappa \\
\mu
\end{array}\right]=\left[\begin{array}{c}
K^{T} \Lambda^{-1} \tau \\
0 \\
0
\end{array}\right] .
$$

The system is solved by inverting the above matrix, for different values of the regularization parameter $\alpha$. This parameter $\alpha$ is chosen a posteriori by studying the trade-off between noise and bias using test cases with synthetic data, see section below.

The noise on the solution can be obtained by noticing that there exist two matrices $V$ and $W$ such that $v=V \tau$ and $w=W \tau$. The errors on the inferred flow components are then given by

$$
\begin{aligned}
& \operatorname{Var} U_{\theta}(r, \theta)=\phi(r, \theta)^{T} V \Lambda V^{T} \phi(r, \theta) \\
& \operatorname{Var} U_{r}(r, \theta)=\phi(r, \theta)^{T} W \Lambda W^{T} \phi(r, \theta),
\end{aligned}
$$

where $\phi(r, \theta)$ denotes the vector of basis functions.

\subsubsection{Tests with synthetics}

We adopt the flexible framework from Liang et al. (2018) (Sect. 4.1) to construct flow models to test the inversions. Here we consider two models, a single-cell model and double-cell model. The colatitudinal component of the velocity is of the form $U_{\theta}(r, \theta)=$ $F(r) G(\theta)$ with $G(\theta)=\sin \theta \sin 2 \theta$. The radial profiles $F(r)$ are constructed by choosing some values $F_{i}$ at fixed points $r_{i}$ and connecting them by cubic spline interpolation (see Table 3.2 and Appendix A of Liang et al. (2018)). The radial component $U_{r}$ is determined through mass conservation and the condition that the flow does not cross the convection zone boundary. The resulting flows are scaled so that the maximum value of $U_{\theta}$ at the surface is $15 \mathrm{~m} / \mathrm{s}$.

To validate the inversion method and choose the regularization parameters in order to be able to reconstruct both the single- and double-cell meridional flow profiles, we generate synthetic travel times with realistic noise. The travel times are obtained using the sensitivity kernels and adding Gaussian noise with zero-mean and the appropriate covariance matrix. The noise level corresponds to either 3 or 11 years of observations. To 
choose the regularization parameter $\alpha$, we investigate the trade-off between bias and propagated noise for a wide range of regularization parameters. Using 900 realizations of the noise, we construct the average inverted flow profile and the corresponding variance. As seen in Figure 3.16 on radial cuts at $30^{\circ}$ latitude, the selected choices of regularization parameters $\alpha_{3}$ and $\alpha_{11}$ are capable of distinguishing between the two models. The bias is approximately the same for the two regularizations, the difference is in the noise level. The inversions perform well also in the case when only distances $\Delta \leq 30^{\circ}$ are used (Figure 3.17). The large-distance travel times do not contribute significantly to the inversions because they have large error bars and the deep flow is determined by the shorter-distance travel times given the constraint of mass conservation.

\subsubsection{Inversions of observed travel times}

Inversions for different seasons. The meridional flow inversions for each season (stream functions) are shown in Figure 3.18. The flow has a single-cell structure in all four seasons, confirming that the correction for the $0.08^{\circ}$ error in the Carrington element describing the inclination angle of the sun's rotation axis is adequate.

Inversions for different sets. We saw earlier that the GONG++ and MDI (rightside up) travel times are in excellent agreement for distances less than $30^{\circ}$ and days in common. Figure 3.13 A shows the inverted stream functions, which indicate that the flows are also in excellent agreement. On the other hand, the bias between HMI and GONG++ travel times seen in Figure 3.1 leads to incompatible flow solutions, see Figure 3.13B. We note that the GONG++ solutions for the period covered by MDI and for the period covered by HMI look very similar.

\subsubsection{Flux-transport dynamo model}

In modeling the effect of the time-dependent meridional circulation on the subsurface toroidal magnetic field, we follow the approach detailed in Cameron and Schüssler (2017). In this framework the evolution of the toroidal field is determined by the one-dimensional mean-field MHD equation assuming that the source term for the toroidal field is the winding up of poloidal field by differential rotation. The evolution equation for the subsurface toroidal flux density $b=\int_{\mathrm{cz}} B_{\phi} r \mathrm{~d} r$ is

$$
\begin{aligned}
\frac{\partial b}{\partial t}= & \sin \theta R_{\odot}^{2} B_{r}\left(\Omega_{\mathrm{S}}-\Omega_{\mathrm{NSSL}}\right) \\
& -\frac{\partial \Omega_{\mathrm{NSSL}}}{\partial \theta} \int_{0}^{\theta} \sin \theta R_{\odot}^{2} B_{r} \mathrm{~d} \theta-\frac{1}{R_{\odot}} \frac{\partial}{\partial \theta}\left(V_{\theta} b\right) \\
& +\frac{\eta}{R_{\odot}^{2}} \frac{\partial}{\partial \theta}\left[\frac{1}{\sin \theta} \frac{\partial}{\partial \theta}(b \sin \theta)\right]-\frac{b}{t_{\mathrm{d}}}
\end{aligned}
$$

where $B_{r}$ is the radial magnetic field at the surface, $\Omega_{\mathrm{S}}$ is the rotation rate at the surface, $\Omega_{\mathrm{NSSL}}$ is the rotation rate at the base of the near-surface shear layer, $\eta$ is the turbulent diffusivity (here we use $100 \mathrm{~km}^{2} / \mathrm{s}$ ), and $t_{\mathrm{d}}=22 \mathrm{yr}$ is the decay time of the toroidal field due to, for example, flux loss through the photosphere (Cameron et al. 2018). Our results 
are rather insensitive to the choice of $t_{\mathrm{d}}$. We assume that the poloidal field lines turn over near the base of near-surface shear layer at $r=0.95 R_{\odot}$. The relevant part of the meridional velocity, $V_{\theta}$ is given by

$$
V_{\theta}=R_{\odot} \overline{U_{\theta} / r},
$$

where the overbar is a radial average weighted by $B_{\phi}$ over the range where it is stored (Cameron and Schüssler 2017). The radial field measurements are taken from a combination of KPVT/NSO and SOLIS synoptic observations extended using HMI synoptic observations and cover the time from 1974 on. We began the simulations from $b=0$ in 1974, and use the average of $V_{\theta}$ over the two cycles for the period before 1996, after which we use the meridional flow $U_{\theta}$ measured in this paper for cycles 23 and 24 .

To determine $V_{\theta}$, the depth range over which the toroidal field is stored is needed. Since this is poorly known, we performed a parameter study and found that using the depth range between the bottom of the convection zone and $0.8 R_{\odot}$ provided a good match with the observations. This depth range is roughly consistent with the range where Hotta (2017) argues that the solar convection zone is subadiabatic and is therefore likely to be a good place to store toroidal field. 


\subsection{Supplementary materials: Supplementary figures}

GONG-Classic and GONG++ 1996.05-2008.04

(3238 days used)
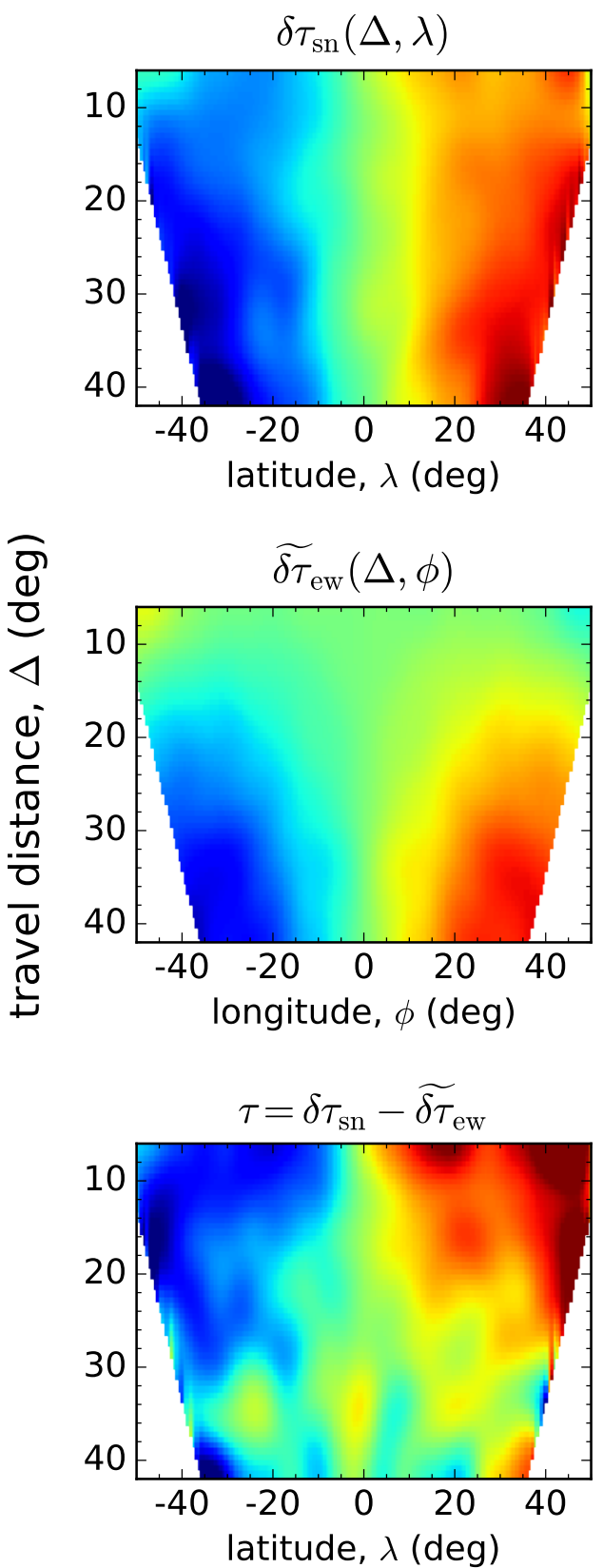

GONG++ 2008.05-2019.04

(3506 days used)

$\delta \tau_{\text {sn }}(\Delta, \lambda)$
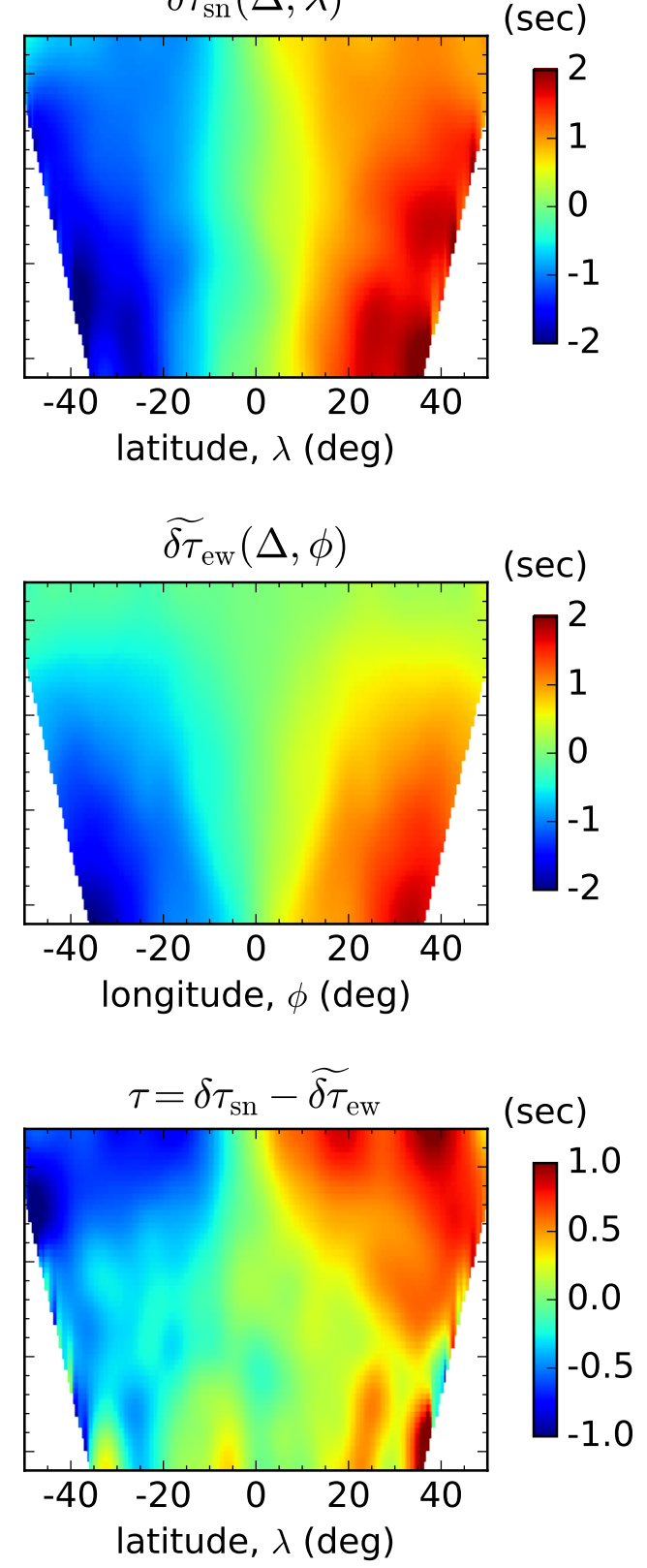

Figure 3.5: GONG travel times averaged over each cycle as a function of distance and latitude/longitude. Top: south-north travel times. Middle: antisymmetrized east-west travel times, representing the center-to-limb variations. Bottom: center-to-limb corrected travel times. All maps are smoothed for clarity. The color scale for each row is shown on the right. 

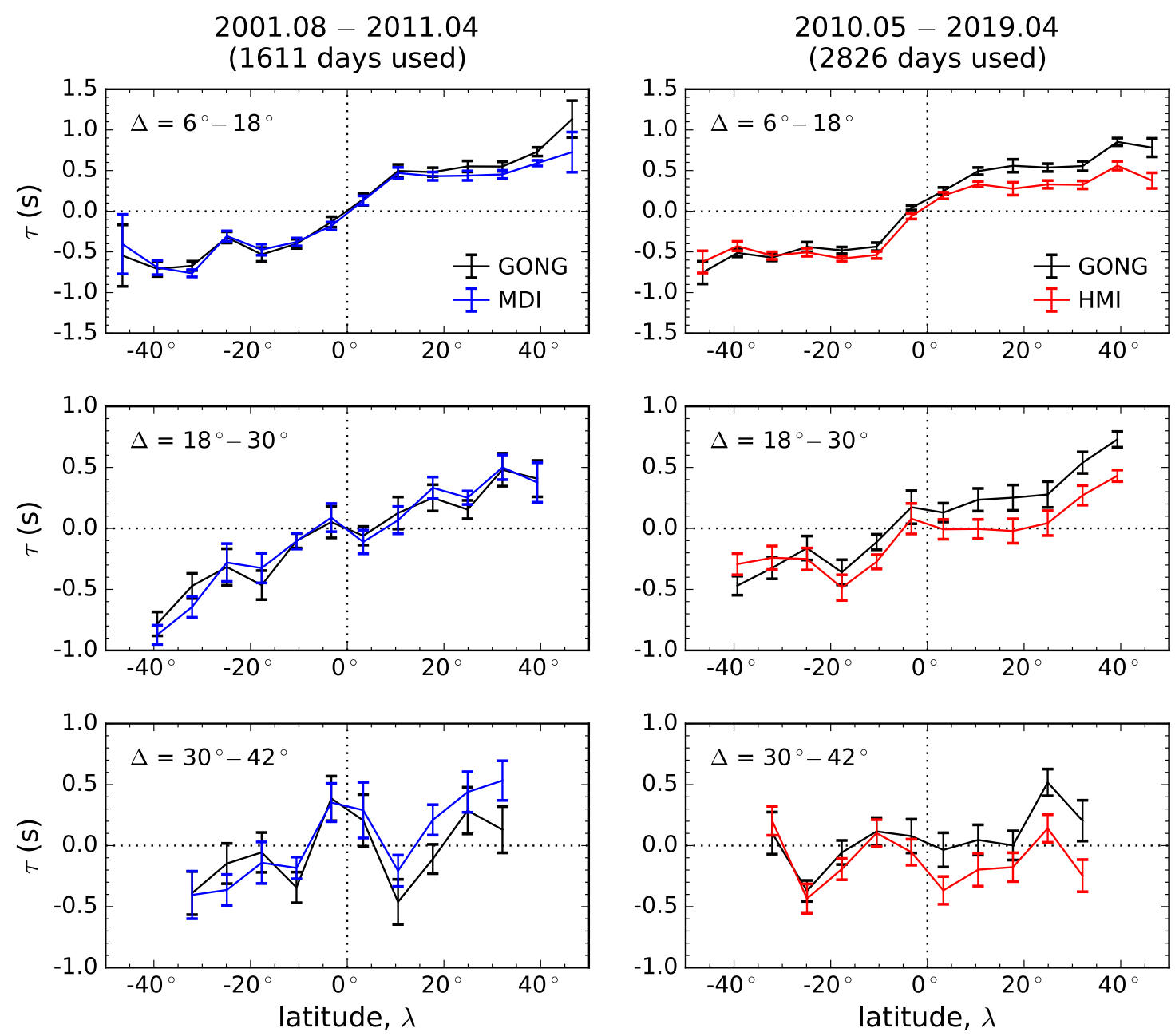

Figure 3.6: Comparison of measured travel times during the overlap periods, GONG++ vs. MDI (left panels) and GONG++ vs. HMI (right panels). Only the days when both data sets are available are used. 


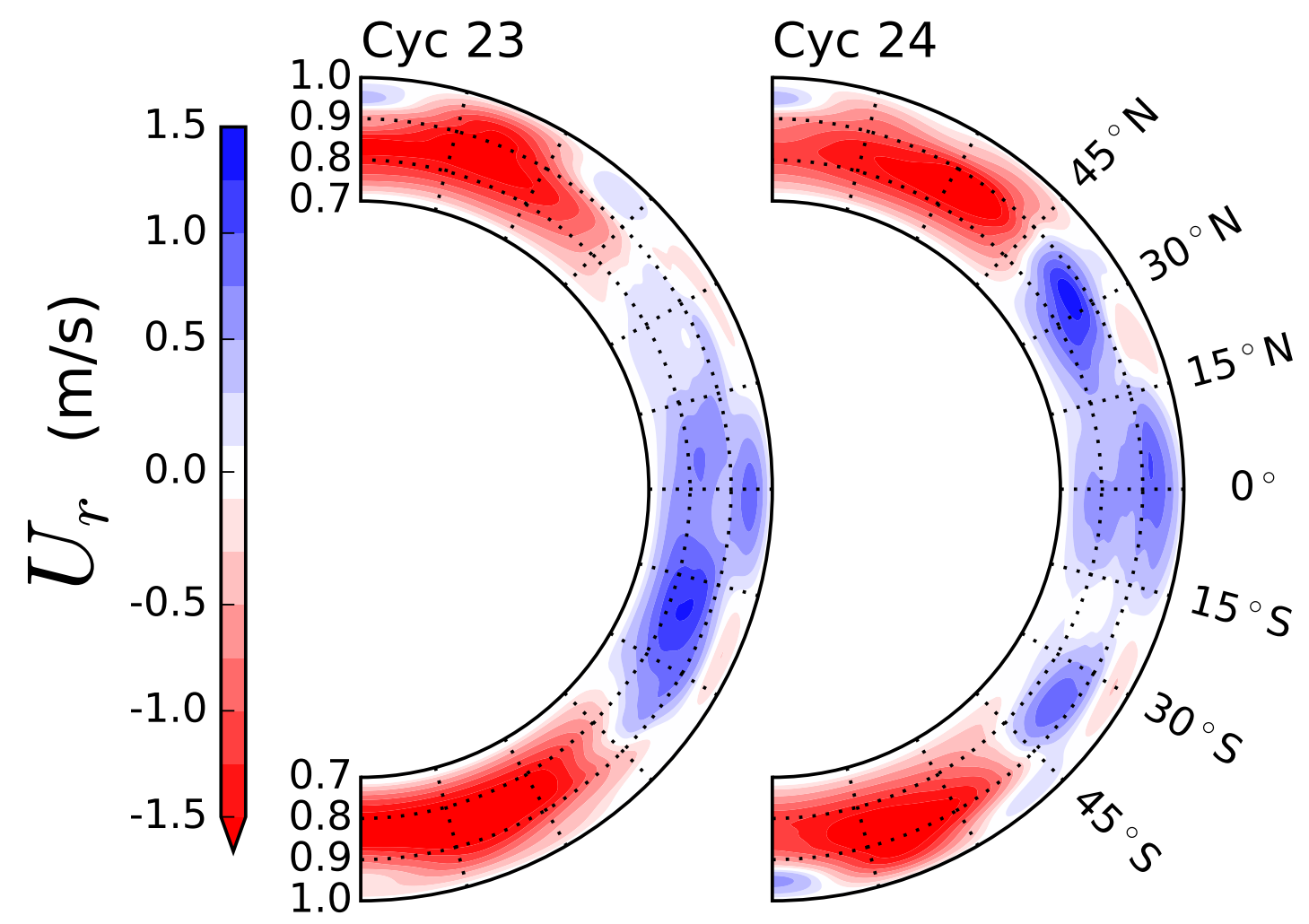

Figure 3.7: Inversion for the radial velocity $U_{r}$ using MDI (first seven years) and GONG data, for each cycle.
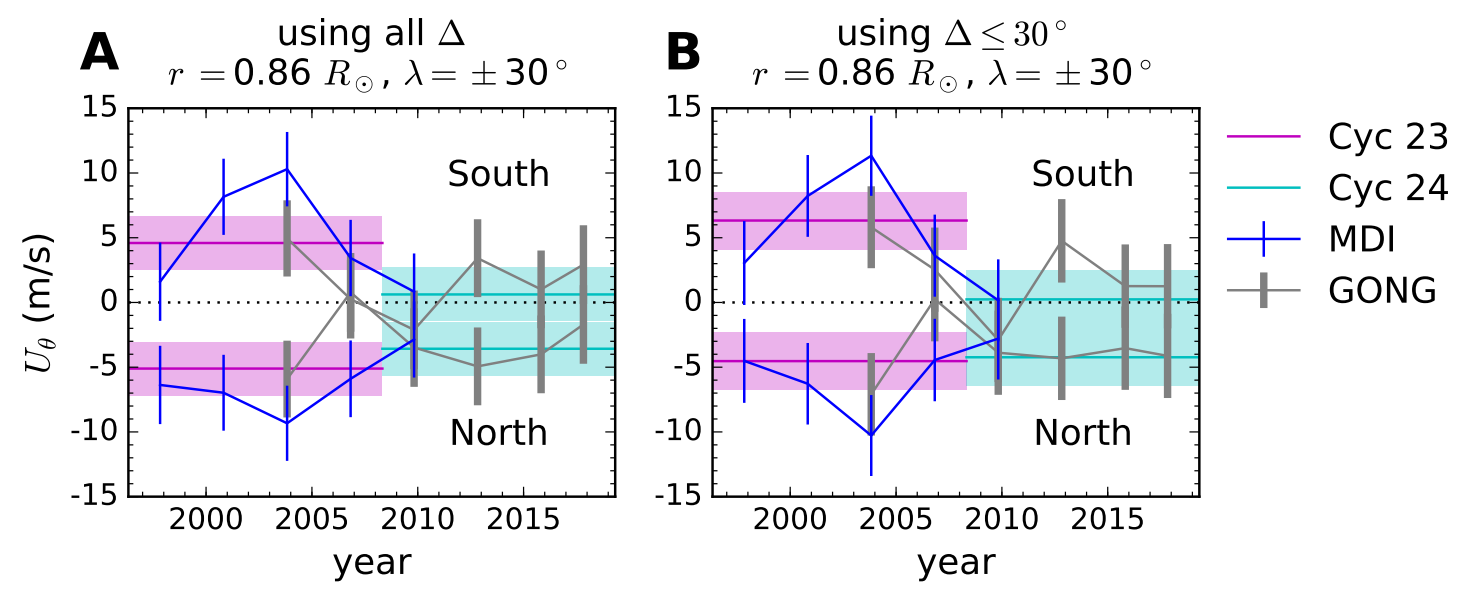

Figure 3.8: Cuts through the solution shown in Figure 3.2 $\mathrm{A}$ and $3.2 \mathrm{C}$ at radius $0.86 R_{\odot}$ and latitudes $\lambda= \pm 30^{\circ}$. 

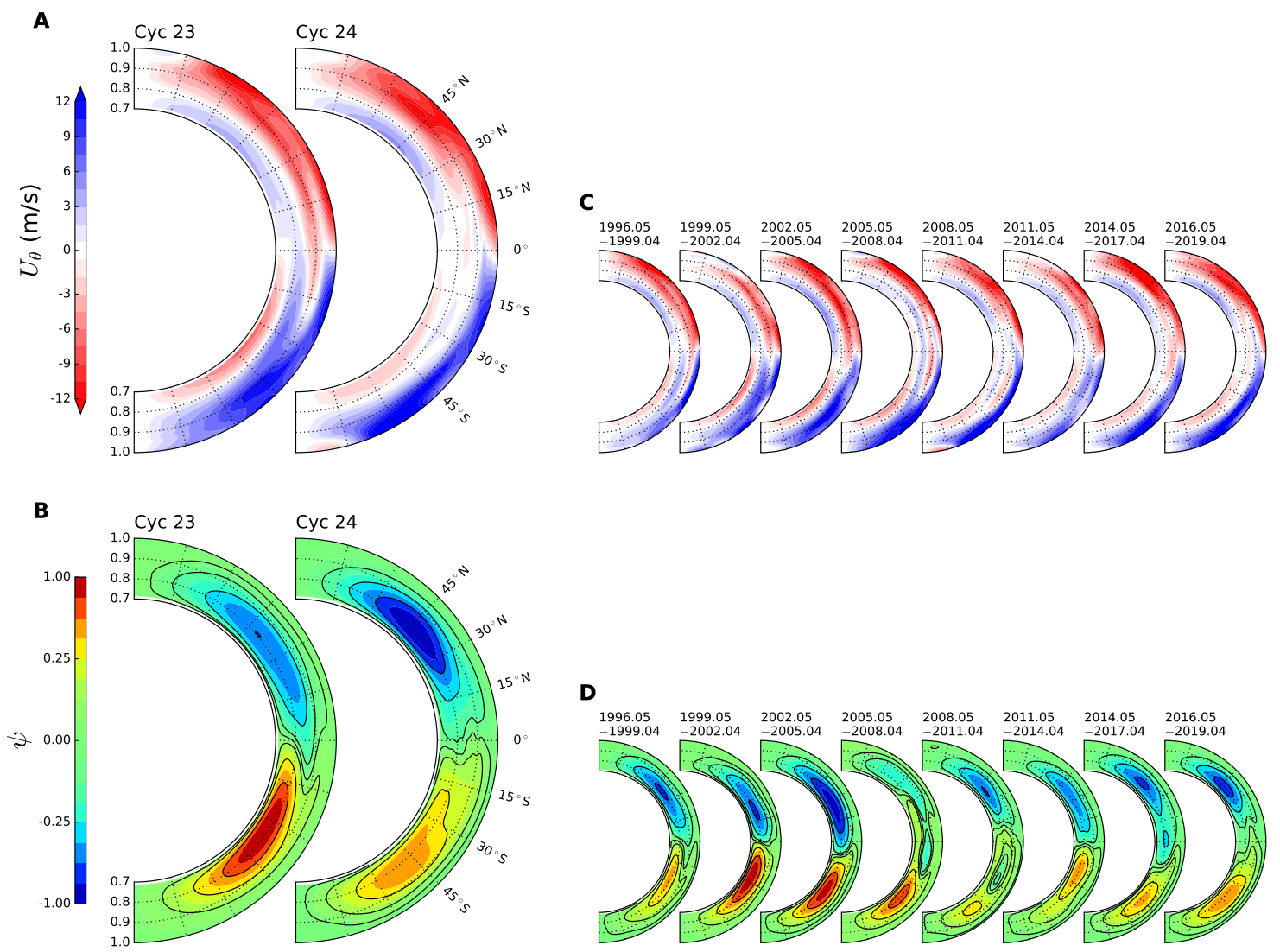

Figure 3.9: Same as Figure 3.2 but using $\Delta \leq 30^{\circ}$ only.
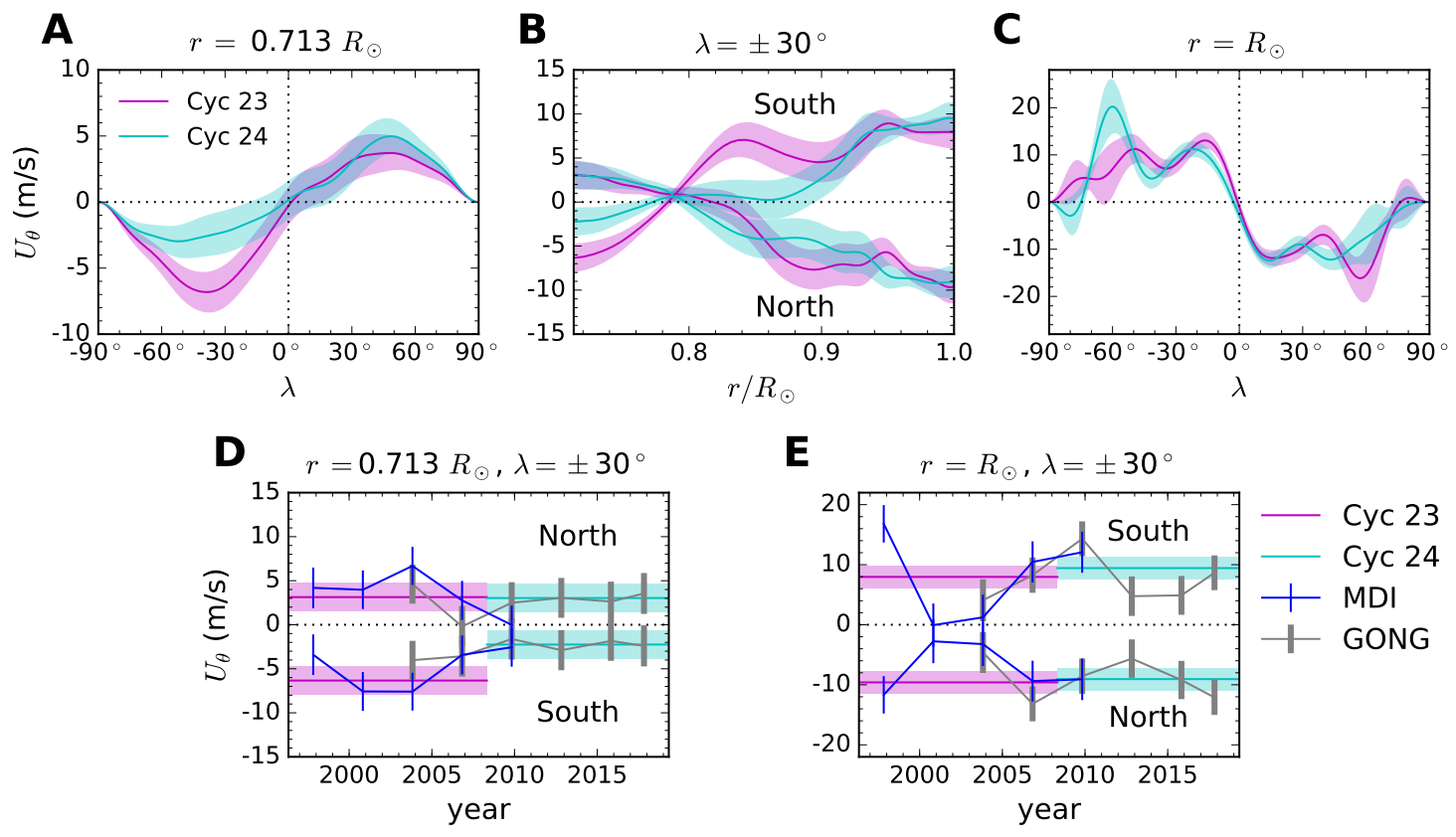

Figure 3.10: Same as Figure 3.3 but using $\Delta \leq 30^{\circ}$ only. 


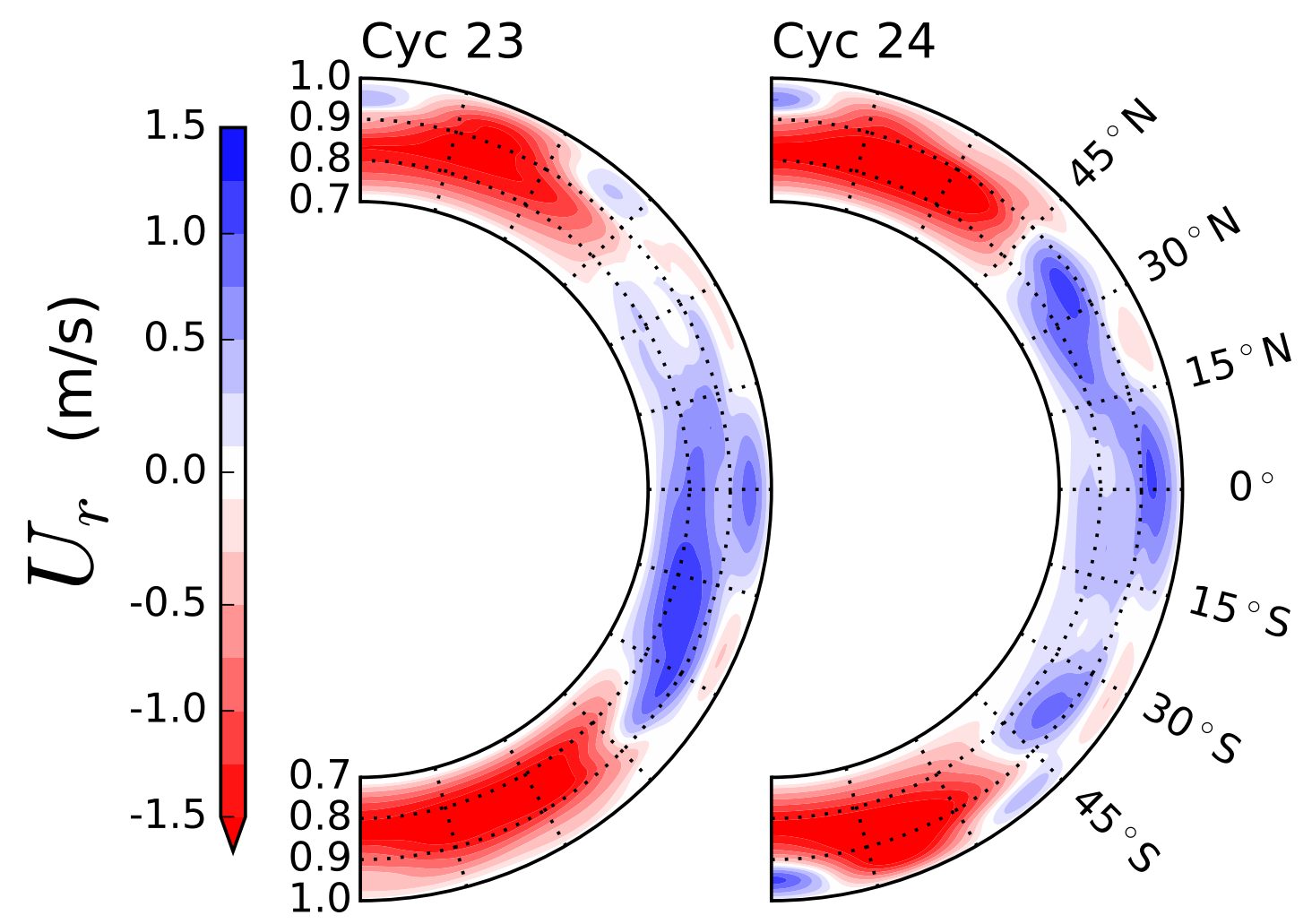

Figure 3.11: Same as Figure 3.7 but using $\Delta \leq 30^{\circ}$ only. 


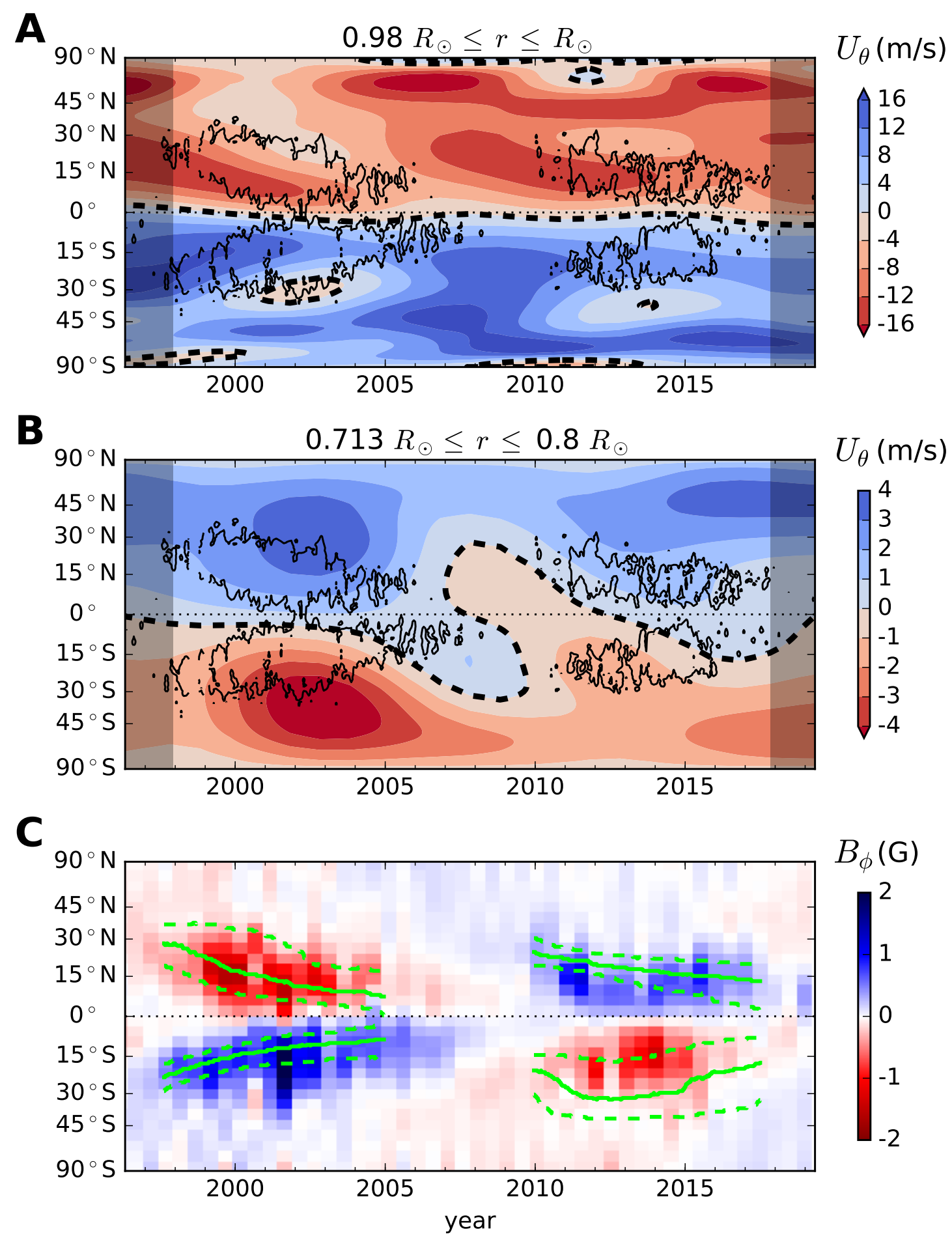

Figure 3.12: Same as Figure 3.4 but $\Delta \leq 30^{\circ}$ only. 

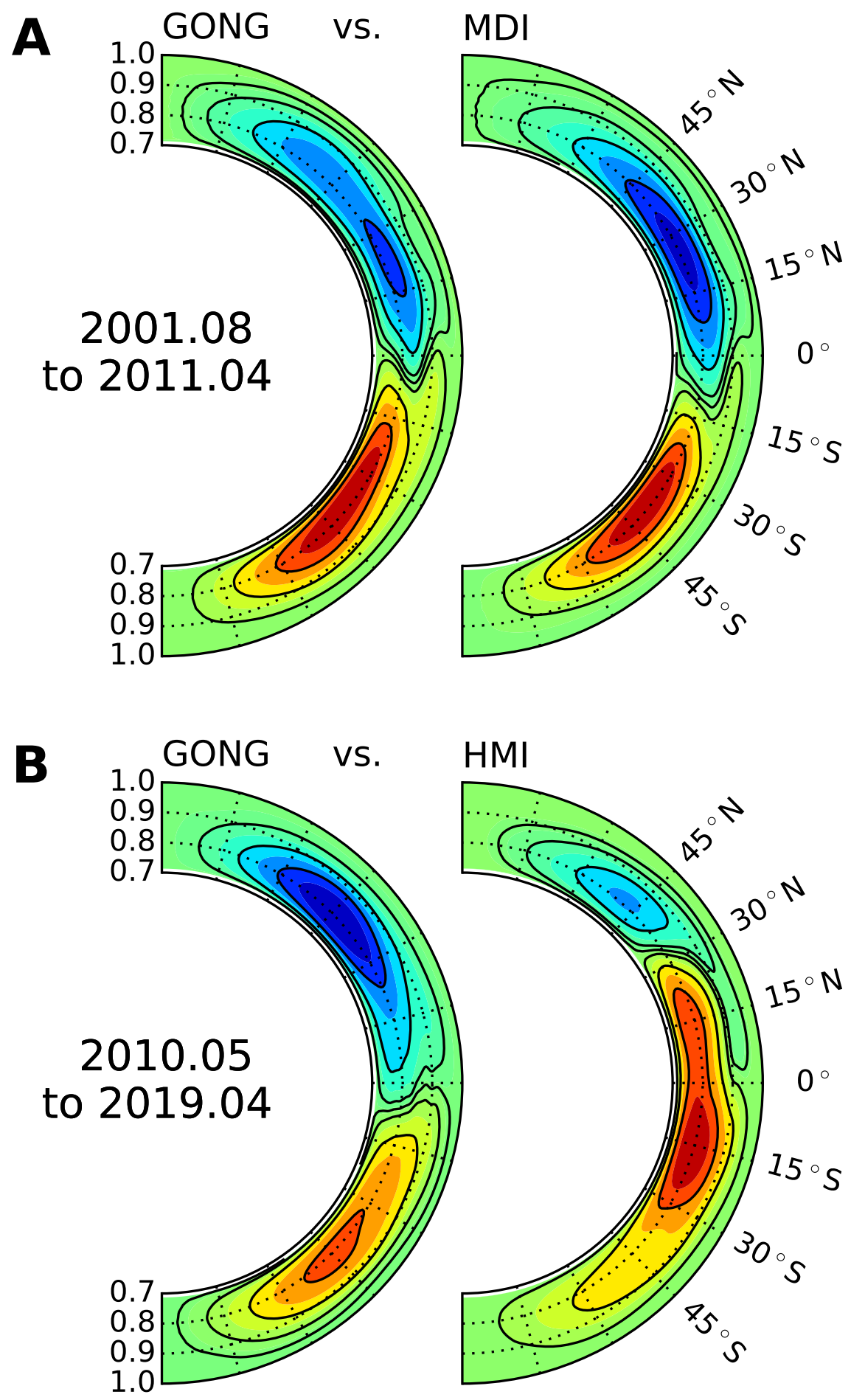

Figure 3.13: Comparison of stream functions $\psi$, inferred from intersecting data sets using $\Delta \leq 30^{\circ}$ only. (A) GONG++ and MDI (August 2001 to April 2011). (B) GONG++ and HMI (May 2010 to April 2019). In each case, only the days during which both data sets are available are used. 

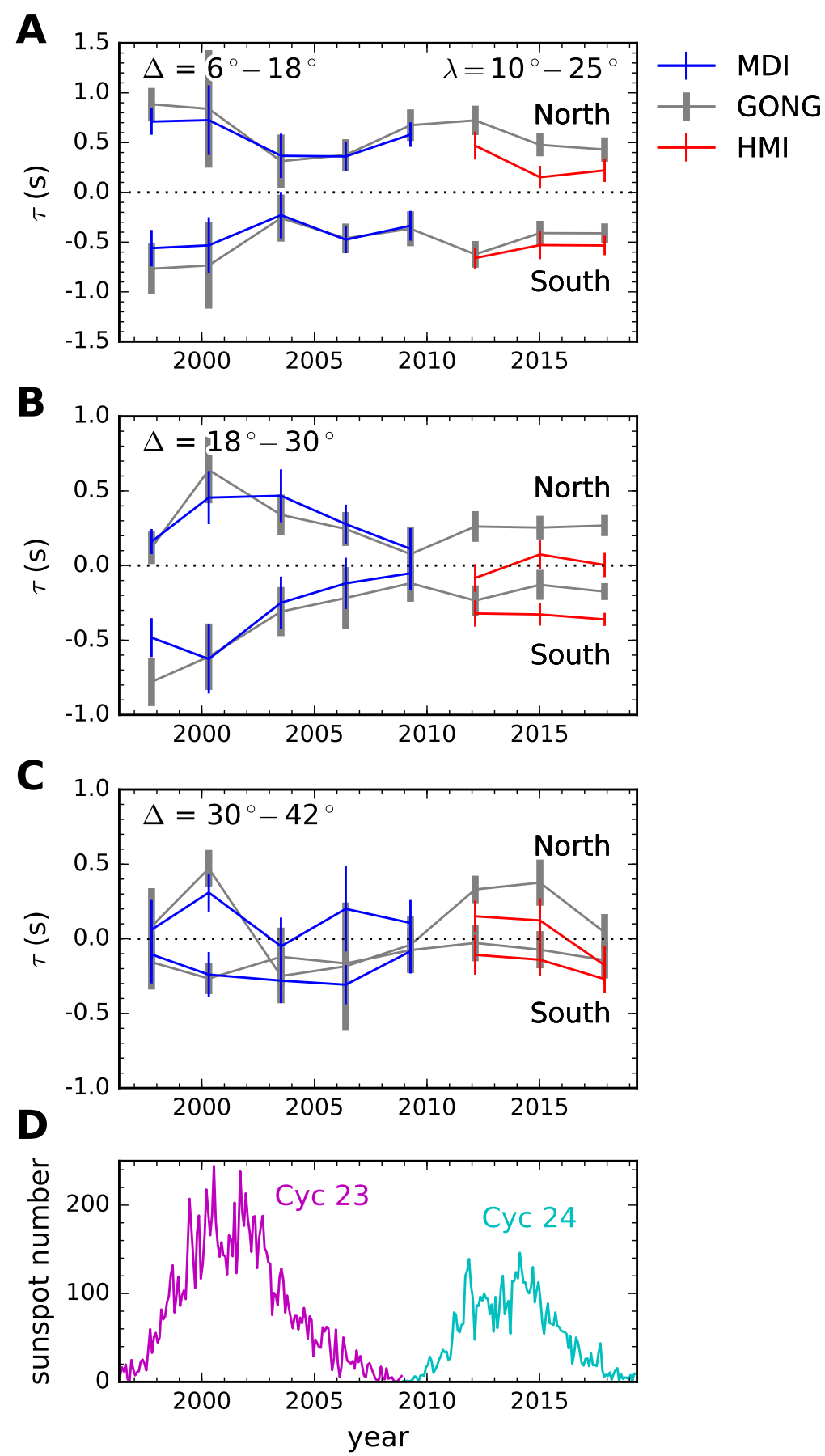

Figure 3.14: Same as Figure 3.1 but including the GONG-Classic data. 

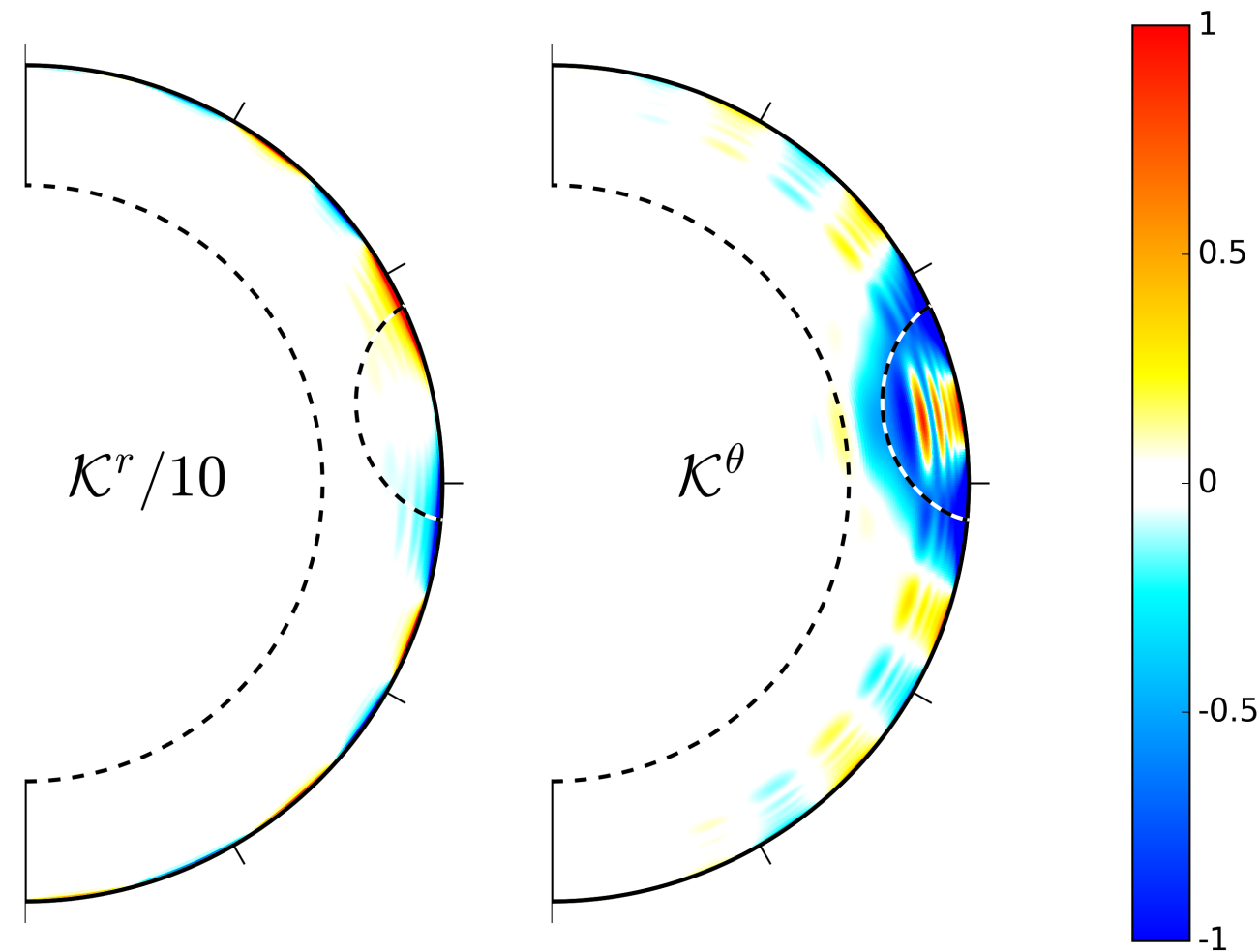

Figure 3.15: Example travel-time sensitivity kernels $\mathcal{K}^{r}$ and $\mathcal{K}^{\theta}$ for a mid-point latitude $\lambda=10^{\circ}$ and a separation distance $\Delta=30^{\circ}$. We show $\mathcal{K}^{r} / 10$ and $\mathcal{K}^{\theta}$ on the same scale, since $U_{r}$ is about an order of magnitude smaller than $U_{\theta}$. The numbers are given in units of $10^{-9} \mathrm{~s}^{2} / \mathrm{m}^{2}$ and only spherical harmonic degrees up to $l=15$ are used. 

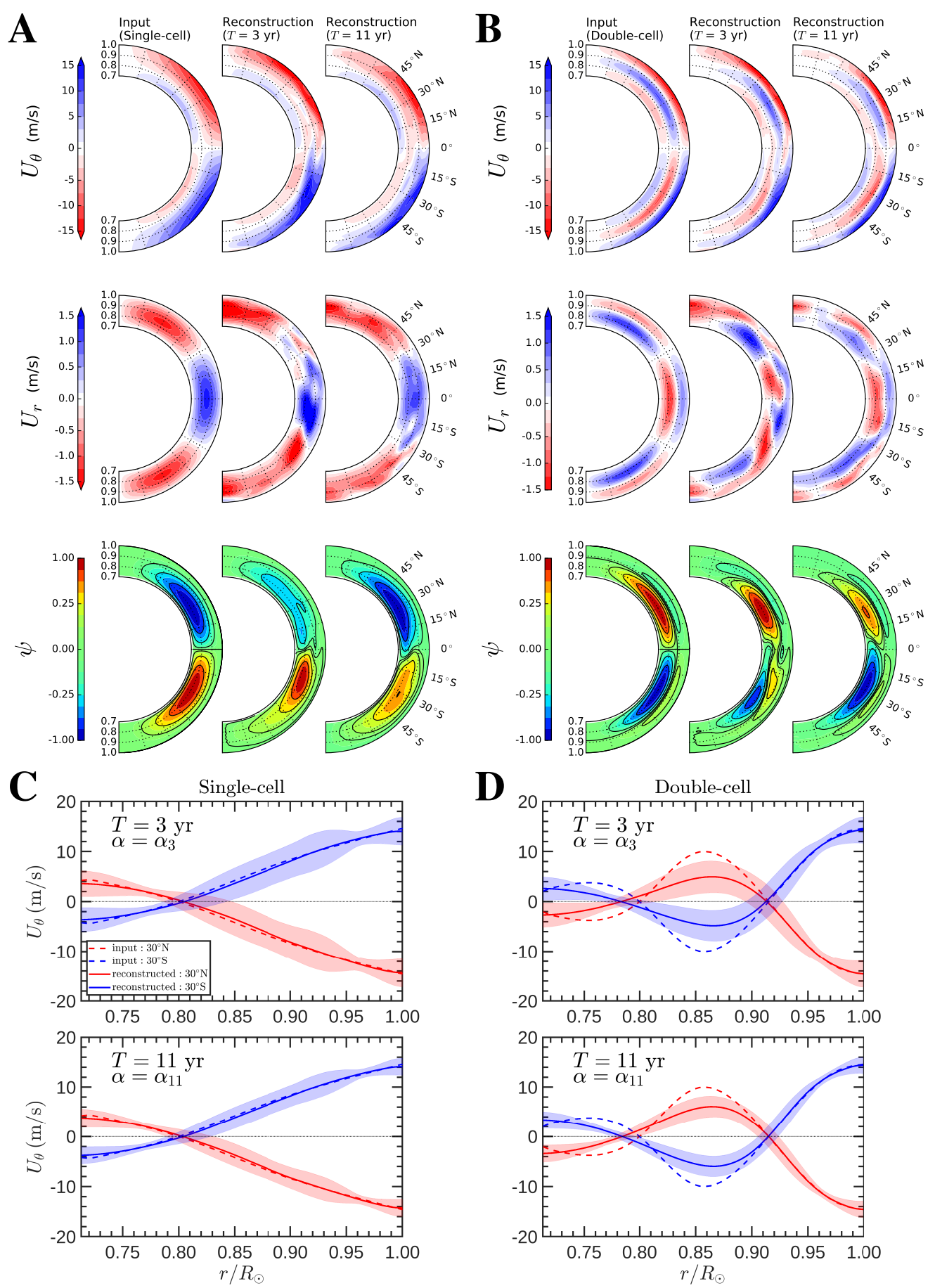

Figure 3.16: Inversion tests using synthetic travel times. (A) Input one-cell meridional flow model (true solution), inferred flow for a three-year noise level (one realization), and inferred flow for an 11-year noise level (one realization). (B) Same as A, but for an input two-cell meridional flow model. (C) Radial cuts of $U_{\theta}$ at $\pm 30^{\circ}$ latitude showing the mean and standard deviation over 900 realizations (solid lines and shading), compared to the input flow profile (dashed curves). (D) Same as $\mathbf{C}$ for the two-cell meridional flow model. 

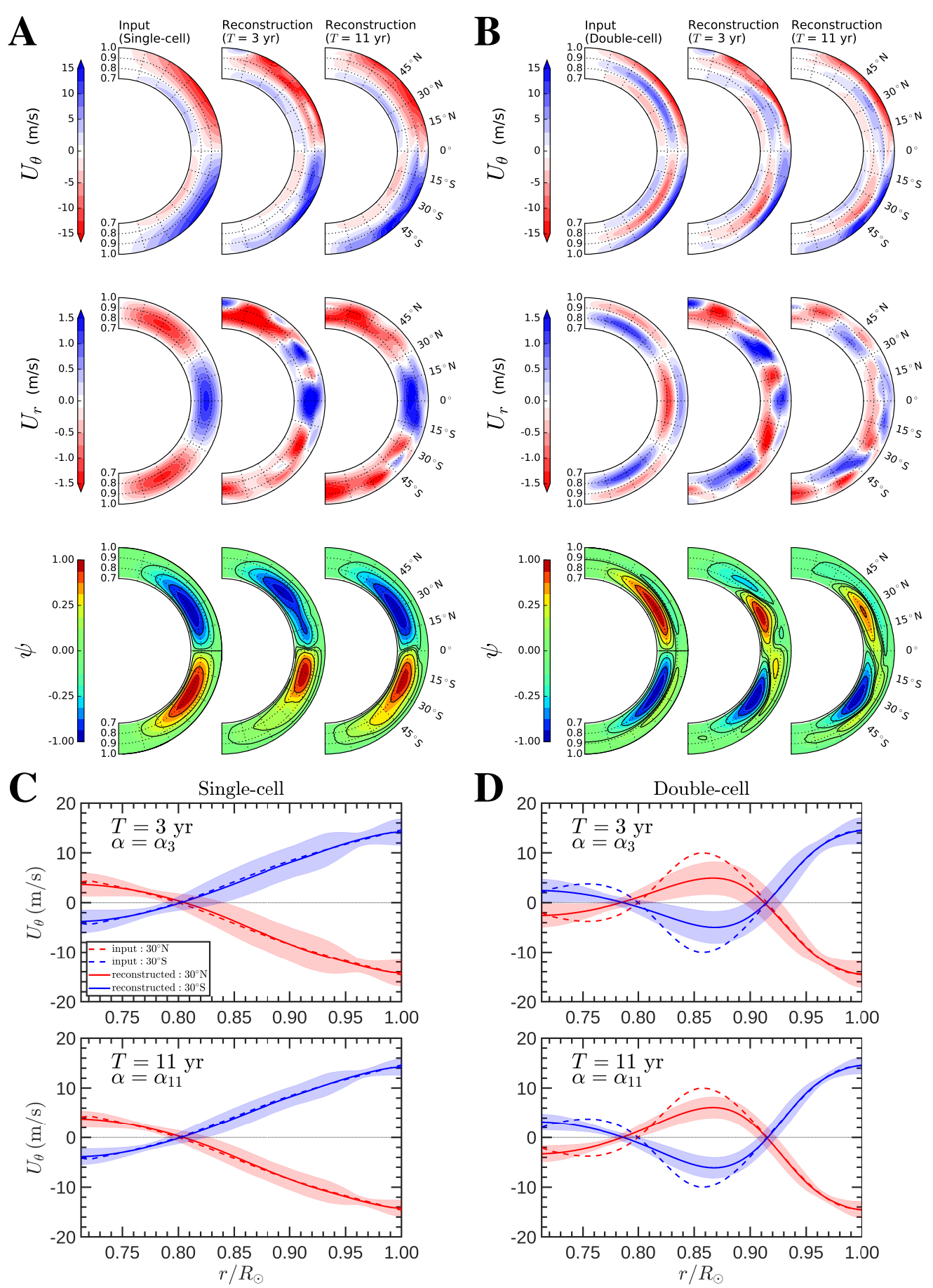

Figure 3.17: Same as Figure 3.16, except that only separation distances $\Delta \leq 30^{\circ}$ were used. The realizations of travel-time noise used to produce panels $\mathbf{A}$ and $\mathbf{B}$ are different than those used for panels $\mathbf{A}$ and $\mathbf{B}$ from Figure 3.16. Panels $\mathbf{C}$ and $\mathbf{D}$ are averages over 900 realizations. 


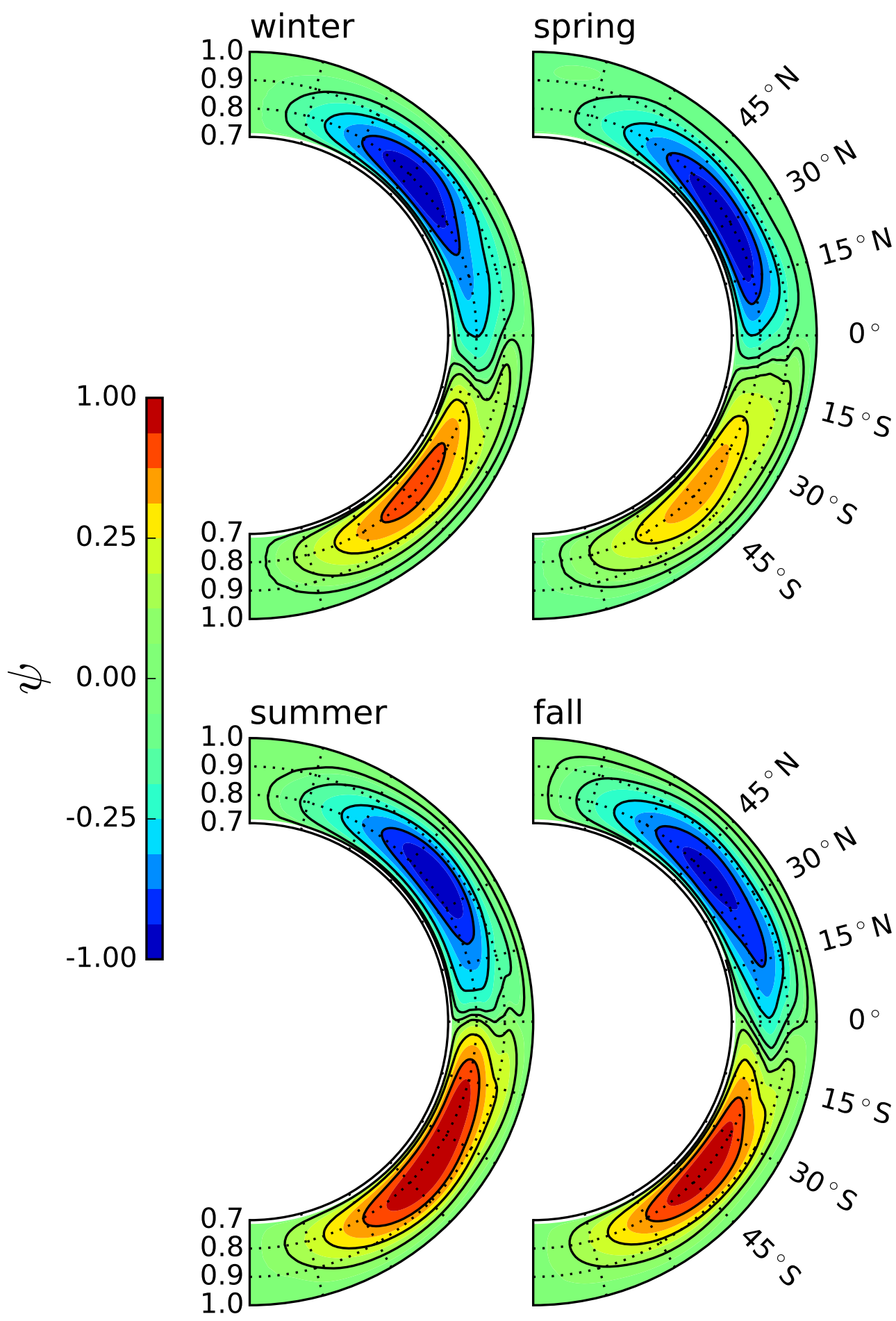

Figure 3.18: Stream function of the inverted flows using individual seasons from 23 years of MDI and GONG++ data, where the months covered by each season are November to January (winter; $\left|B_{0}\right|<4^{\circ}$ ), February to April (spring; $B_{0}<-4^{\circ}$ ), May to July (summer; $\left|B_{0}\right|<4^{\circ}$ ), and August to October (fall; $B_{0}>4^{\circ}$ ). 



\title{
4 Helioseismic inversions for the solar meridional flow using synthetic data ${ }^{[1}$
}

\begin{abstract}
Inconsistent inferences of the solar meridional flow have been reported in the literature, especially for the deeper layers of the convection zone. This is due to differences in the travel-time measurements and in the inversion procedure. Here, we tune and validate helioseismic inversions for the solar meridional flow using synthetic travel times and Born sensitivity kernels. Synthetic travel times are generated with realistic noise which is due to the stochastic nature of solar oscillations. We test the importance of the regularization term as well as the mass conservation and the boundary constraint. We find that the mass conservation constraint is necessary to reconstruct the radial component of the meridional flow and also improves the reconstruction of the horizontal component. To avoid fast flow variations in latitude, the weighted flow vorticity is introduced in the regularization term of the inverse problem. With the same bias in the solution, the weighted flow vorticity regularization matrix leads to smaller propagated errors (approximately two times) to the radial component of the reconstructed flow compared to the other regularization matrices. The results also suggest that imposing that the radial flow does not cross the base of the convection zone is crucial to reconstruct the flow in the deep layers. Additionally, we find that the travel times with separation distances larger than $30^{\circ}$ do not contribute significantly to the inversions because of the large noise of these travel times and the mass conservation constraint.
\end{abstract}

\subsection{Introduction}

There is a fairly good understanding about the near-surface solar meridional flow since the flow has been measured using different methods (e.g. Gizon and Birch 2005, Miesch 2005). However, this is not the case for the deep solar meridional flow. Different authors have reported inconsistent inferences of the solar meridional flow for the deeper layers of the convection zone using time-distance helioseismic inversions. They have reported different geometries (single-cell or double-cell) for the meridional flow in the radial direction (Zhao et al. 2013, Jackiewicz et al. 2015, Rajaguru and Antia 2015, Chen and Zhao

\footnotetext{
${ }^{1}$ This chapter presents work done by M. Pourabdian, D. Fournier, Z.-C. Liang, L. Gizon, and T. Hohage. Contribution statement: MP developed the inversion setup in collaboration with DF. MP accomplished the numerical computations for the inversions and wrote the text.
} 
2017, Böning et al. 2017, Lin and Chou 2018, Mandal et al. 2018). The reasons for the inconsistency in the reported results possibly stem from using different data sets, different observation time, and systematic effects including the center-to-limb effects (e.g. Zhao et al.2012), instrumental misalignment (e.g. Giles et al. 1997, Beck and Giles 2005), and surface magnetic field effects (e.g. Liang and Chou 2015).

In addition to the mentioned possible reasons for the inconsistent inferences of the solar meridional flow, different helioseismic inversion setups may give different solutions which affects the understanding of the total profile of the meridional flow. For instance, the assumption of uncorrelated travel-time measurements may lead to underestimation of the errors in the inversion solution by a factor of approximately $2-4$ (Böning et al. 2017). Moreover, computing travel-time sensitivity kernels using the ray approximation, which is a high-frequency approximation and gives sensitivity only along a ray path may be insufficient (Birch et al. 2001). Instead, using the first-order Born approximation (e.g. Gizon and Birch 2002), which is a single-scattering approximation and provides sensitivities away from the geometrical ray path, does not have this limitation. As another example, the radial component of the solar meridional flow is difficult to infer with timedistance helioseismology (e.g. Giles 2000, Rajaguru and Antia 2015) as it is very smaller than its horizontal counterpart. To be able to infer this component, the solution needs to be constrained by mass conservation. Many other points can be raised regarding the helioseismic inversions, such as how a regularization term would affect the solution or how to choose the regularization parameter. Hence, tuning and validating the inversions is fundamental before using the observational measurements.

In this chapter, we present the procedure and the corresponding test cases to develop an inversion setup for inferences of the solar meridional flow using time-distance helioseismology. We tune and validate the helioseismic inversions using synthetic data. In Section 4.2, the forward problem for the meridional flow and the utilized models are explained. The inversion procedure is described in Section 4.3 . The results of different test cases using synthetic travel times are presented in Section 4.4 and conclusions are summarized in Section 4.5.

\subsection{Forward problem}

In time-distance helioseismology (Duvall et al. 1993), one measures travel times of the wave packets traveling between any two points on the solar surface to infer the physical properties of the solar interior. These travel times are shifted due to the perturbations in the solar interior with respect to a reference model. For instance, let's consider two points on the solar surface like A and B and measure the travel times which take for acoustic waves to travel from A to B and also from B to A. The difference between the two travel times is sensitive to the subsurface meridional flow. In this sense, the southward minus northward travel-time shifts, hereafter travel-time measurements, are used to probe the subsurface meridional flow.

From an observational point of view, the northward and southward travel times are extracted from the averaged cross-covariance function between the oscillation signals (e.g. Doppler velocity observations) by fitting a Gabor wavelet function (Kosovichev and Duvall 1997, Duvall et al. 1997) or using a one-parameter fit (Gizon and Birch 2004) to the 

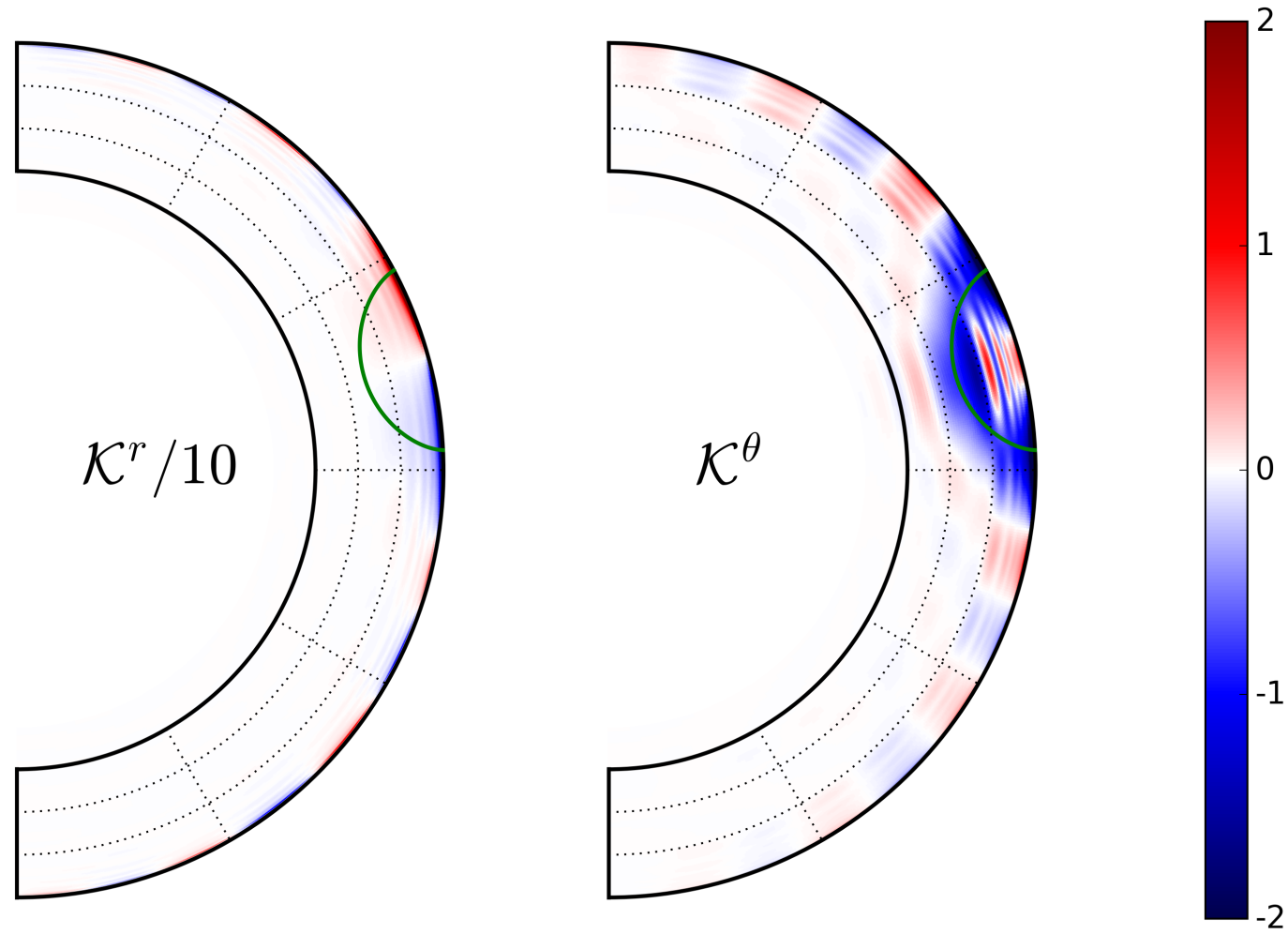

Figure 4.1: Born sensitivity kernels $\mathcal{K}^{r}$ and $\mathcal{K}^{\theta}$ of a travel-time measurement for a pair of points on the solar surface where the mid-point latitude and separation distance between the points are $15.3^{\circ}$ and $25.2^{\circ}$, respectively. The kernels are computed in the spherical harmonic space, up to degrees $l=15$. The units of the kernels are in $10^{-9} \mathrm{~s}^{2} / \mathrm{m}^{2}$. For illustrative purposes, the kernel $\mathcal{K}^{r}$ is shown divided by a factor 10 since $U_{r}$ is about 10 times smaller than $U_{\theta}$. The oscillating bands seen in the kernels are due to the finitewavelength effects that provide sensitivity away from the local ray paths (green curves).

first-skip wave packet in the positive and negative time lags, respectively.

\subsubsection{Modeling travel-time measurements using Born sensitivity ker- nels}

In time-distance helioseismology, the interpretation of the travel-time measurements is based on solving the forward problem that determines the relationship between the traveltime measurements and the subsurface properties such as the meridional flow of interest in this study. Here, this relationship is given by the finite-wavelength Fréchet kernels in the first-order Born approximation. The forward problem of the solar meridional flow is written as

$$
\tau_{i}=\int_{0}^{\pi} \int_{0}^{R_{\odot}}\left(\mathcal{K}_{i}^{r}(r, \theta) U_{r}(r, \theta)+\mathcal{K}_{i}^{\theta}(r, \theta) U_{\theta}(r, \theta)\right) \mathrm{d} r \mathrm{~d} \theta+n_{i}, \quad i \in[1, N]
$$

where $\mathcal{K}^{r}$ and $\mathcal{K}^{\theta}$ are longitudinally averaged sensitivity kernels, $U_{r}(r, \theta)$ and $U_{\theta}(r, \theta)$ are the radial and horizontal components of the meridional flow at 2D spatial location $(r, \theta)$, 
respectively. Here, we work in spherical-polar coordinates where $r$ denotes the distance from the solar center and $\theta$ denotes the colatitude. The index $i$ is associated with traveltime measurements $\left[\tau_{i}\right]$ with noise $\left[n_{i}\right]$

$$
\tau_{i}=\tau\left(\theta_{i}, \Delta_{i}\right), \quad n_{i}=n\left(\theta_{i}, \Delta_{i}\right), \quad i \in[1, N]
$$

where $N$ is the number of measurements and $\left(\theta_{i}, \Delta_{i}\right)$ are coordinates of each travel-time measurement. Each pair of points on the solar surface are separated by an angular distance $\left[\Delta_{i}\right]$ and $\theta_{i}$ denotes the colatitude of the mid-point between the pair of points. Following the framework of Liang et al. (2018), the travel distances $\left[\Delta_{i}\right]$ range from $6^{\circ}$ to $42^{\circ}$ in increments of $0.6^{\circ}$ while the colatitudes are in the range $30^{\circ}<\theta_{i}<150^{\circ}$ every $0.6^{\circ}$. The number of measurements get smaller for larger distances as both points have to be in the range $\left[30^{\circ}, 150^{\circ}\right]$.

The computation of the kernels follows the framework of Gizon et al. (2017). It requires the knowledge of Green's functions obtained from a wave equation with a solar background model (e.g. the standard solar model S described by Christensen-Dalsgaard et al. (1996)). The Green's functions are computed using the finite element method with the solver Montjoie (Chabassier and Durufle 2016). In order to have a modeled power spectrum as close as possible to the observed one, we use the same filters in the kernel computation as in the measurement procedure. Moreover, the measured linewidths of the solar power spectrum are used to tune the attenuation in the wave equation. To accelerate the computation of the kernels, we use the method proposed by Fournier et al. (2018). Examples of the sensitivity kernels $\mathcal{K}^{r}$ and $\mathcal{K}^{\theta}$ of a travel-time measurement are shown in Figure 4.1.

\subsubsection{Discretization of the model}

The meridional flow components are expressed as linear combinations of basis functions as explained in Section 3.2.2. Synthetic travel times introduced in Equation 4.1 are set in a vector,

$$
\tau=\left[\tau_{1} \tau_{2} \ldots \tau_{N}\right]^{T}
$$

where $T$ denotes the transpose of a vector/matrix. The flow coefficients $w_{j}$ and $v_{j}$, introduced in Equations 3.4 and 3.5 , are also sorted in a vector of length $2 M$ given as

$$
\boldsymbol{u}=\left[w_{1} w_{2} \ldots w_{M} v_{1} v_{2} \ldots v_{M}\right]^{T},
$$

where $M$ is the number of flow coefficients for each flow component. Using the decomposition of the flow in Equations 3.4 and 3.5 , the linear equation introduced in Equation 4.1 is written in matrix form as,

$$
\tau=K \boldsymbol{u}+\boldsymbol{n}
$$

where $\boldsymbol{n}=\left[n_{1} n_{2} \ldots n_{N}\right]^{T}$ is the corresponding noise vector of the travel times. The matrix of kernel coefficients [ $K]$, of size $N \times 2 M$, contains the projection of the kernels onto the basis functions. The elements of this matrix is obtained from Equation 3.9 (Section 3.2.3). 


\subsubsection{Noise}

Due to the stochastic excitation of solar oscillations, travel-time measurements are noisy so we add a noise term to our synthetic travel times. In this study, we assume the noise is Gaussian with zero mean and a realistic solar noise level, which is specified by the noise covariance matrix $[\Lambda]$. The elements of the noise covariance matrix is obtained from Equation 3.7 (Section 3.2.3).

This $N \times N$ matrix is computed using the theory from Fournier et al. (2014) that only requires the knowledge of the expectation value of the cross-covariance for the reference 1D solar model. Using the convenient source of excitation introduced in Gizon et al. (2017), the cross-covariance function is then directly related to the imaginary part of the Green's function.

Due to the foreshortening effect, the observational errors increase towards the limb and this effect is not considered in the model by Fournier et al. (2014). Hence, we scale the noise covariance matrix with a realistic noise level from the observations to take care of the latitudinal dependence of the noise.

\subsection{Inversion setup}

To infer the subsurface meridional flow in the solar interior, the travel-time measurements need to be inverted. Using the regularized least squares (RLS) method (e.g. Kosovichev 1996, Tarantola 2005), we seek to minimize the cost function

$$
\left\|\Lambda^{-1 / 2}(K \boldsymbol{u}-\tau)\right\|^{2}+\alpha\|D \boldsymbol{u}\|^{2}
$$

with respect to flow coefficients $\boldsymbol{u}$ where $\|\cdot\|$ denotes the discrete $L^{2}$ norm. The second term in Equation 4.6 adds a regularization term to the misfit function $\left\|\Lambda^{-1 / 2}(K \boldsymbol{u}-\tau)\right\|^{2}$ with a regularization matrix $[D]$ and a regularization parameter $[\alpha]$. The regulation term reduces the propagation of the noise from the travel-time measurements. The regularization parameter is tuned to obtain the best trade-off between the misfit to the data and the smoothness of the solution.

\subsubsection{Regularization term}

The choice of the regularization matrix $[D]$ in the regularization term $\alpha\|D \boldsymbol{u}\|^{2}$ is important in RLS. In this study, we consider several choices for $D$.

\subsubsection{Flow vorticity $(\nabla \times U)_{\varphi}$ and weighted flow vorticity}

Denoting $\boldsymbol{U}=\left(U_{r}(r, \theta), U_{\theta}(r, \theta)\right)$, one option for the regularization operator is that $D \boldsymbol{u}$ is the discretization of the flow vorticity or another option is $D \boldsymbol{u}$ is the discretization of the weighted flow vorticity. The equation for flow vorticity is

$$
(\boldsymbol{\nabla} \times \boldsymbol{U})_{\varphi}=\frac{1}{r}\left(\frac{\partial}{\partial r}\left(r U_{\theta}(r, \theta)\right)-\frac{\partial U_{r}(r, \theta)}{\partial \theta}\right)
$$


where $\varphi$ denotes the azimuthal direction in spherical-polar coordinates. We also introduce the equation for the weighted flow vorticity as:

$$
\frac{1}{r}\left(\frac{\partial}{\partial r}\left(r U_{\theta}(r, \theta)\right)-\frac{\beta \partial U_{r}(r, \theta)}{\partial \theta}\right)
$$

where $\beta$ is a factor to compensate the fact that $U_{r}$ is smaller than $U_{\theta}$. In this study, we consider $\beta=10$ in the case of the weighted flow vorticity unless it is specified by another value in this chapter. The factor $\beta=10$ accounts for the fact that $U_{r}$ is about 10 times smaller than $U_{\theta}$. We study the effect of this factor on the inversions in Section 4.4.3. We also define the scalar product for any two functions $f(r, \theta)$ and $g(r, \theta)$ as

$$
\langle f, g\rangle=\int_{0}^{\pi} \int_{r_{b}}^{R_{\odot}} f(r, \theta) g(r, \theta) r^{2} \sin \theta \mathrm{d} r \mathrm{~d} \theta,
$$

where $r_{b}=0.713 R_{\odot}$ denotes the radius at the base of the convection zone (ChristensenDalsgaard et al.1991).

By projecting the equation for the weighted flow vorticity (Equation 4.8) onto the basis functions with respect to the scalar product, we obtain the weighted flow vorticity in matrix form $D \boldsymbol{u}$ where $D$ is the weighted flow vorticity regularization matrix of size $M \times 2 M$ and has elements

$$
\begin{aligned}
D_{i, j} & =\left\langle\phi_{i},-\frac{\beta}{r} \frac{\partial \phi_{j}}{\partial \theta}\right\rangle, \\
D_{i, j+M} & =\left\langle\phi_{i}, \frac{1}{r} \frac{\partial\left(r \phi_{j}\right)}{\partial r}\right\rangle,
\end{aligned}
$$

with $1 \leq i, j \leq M$. For obtaining the flow vorticity in matrix form $D \boldsymbol{u}$ where $D$ is the flow vorticity regularization matrix, we only need to choose $\beta=1$ in Equation 4.10.

\subsubsection{Flow gradient $\nabla U$}

Another choice for the regularization term is that $D \boldsymbol{u}$ is the discretization of the flow gradient. The gradient of the radial and horizontal components of the flow with respect to the $r$ and $\theta$ variables is written in the form

$$
\boldsymbol{\nabla} \boldsymbol{U}=\left[\begin{array}{cc}
\frac{\partial U_{r}(r, \theta)}{\partial r} & \frac{\partial U_{\theta}(r, \theta)}{\partial r} \\
\frac{1}{r} \frac{\partial U_{r}^{r}(r, \theta)}{\partial \theta} & \frac{1}{r} \frac{\partial U_{\theta}(r, \theta)}{\partial \theta}
\end{array}\right] .
$$

By projecting Equation 4.12 onto the basis functions in the radial and horizontal directions, we obtain the flow gradient in matrix form $D \boldsymbol{u}$ where the flow gradient regularization matrix $[D]$, of size $2 M \times 2 M$, consists of four sub-matrices as

$$
D=\left[\begin{array}{ll}
D^{1} & D^{1} \\
D^{2} & D^{2}
\end{array}\right]
$$

The sub-matrices $D^{1}$ and $D^{2}$ have elements

$$
\begin{aligned}
& D_{i, j}^{1}=\left\langle\phi_{i}, \frac{\partial \phi_{j}}{\partial r}\right\rangle, \\
& D_{i, j}^{2}=\left\langle\phi_{i}, \frac{1}{r} \frac{\partial \phi_{j}}{\partial \theta}\right\rangle,
\end{aligned}
$$

with $1 \leq i, j \leq M$. 


\subsubsection{Flow Laplacian $\nabla^{2} U$}

We also consider $D \boldsymbol{u}$ to be the discretization of the flow Laplacian. The equation for the flow Laplacian is

$$
\begin{aligned}
\boldsymbol{\nabla}^{2} \boldsymbol{U} & =\left[\frac{1}{r^{2}} \frac{\partial}{\partial r}\left(r^{2} \frac{\partial U_{r}(r, \theta)}{\partial r}\right)+\frac{1}{r^{2} \sin \theta} \frac{\partial}{\partial \theta}\left(\sin \theta \frac{\partial U_{r}(r, \theta)}{\partial \theta}\right)\right] \mathbf{e}_{r} \\
& +\left[\frac{1}{r^{2}} \frac{\partial}{\partial r}\left(r^{2} \frac{\partial U_{\theta}(r, \theta)}{\partial r}\right)+\frac{1}{r^{2} \sin \theta} \frac{\partial}{\partial \theta}\left(\sin \theta \frac{\partial U_{\theta}(r, \theta)}{\partial \theta}\right)\right] \mathbf{e}_{\theta},
\end{aligned}
$$

where $\mathbf{e}_{r}$ and $\mathbf{e}_{\theta}$ are the unit vectors in spherical-polar coordinates.

With the same procedure and projecting the equation for the flow Laplacian onto the basis functions, we obtain the flow Laplacian in matrix form $D \boldsymbol{u}$, and the flow Laplacian regularization matrix $[D]$ of size $2 M \times 2 M$ is

$$
D=\left[\begin{array}{ll}
L & 0 \\
0 & L
\end{array}\right]
$$

The sub-matrix $L$ has elements

$$
L_{i, j}=\left\langle\phi_{i},\left(\frac{\partial^{2} \phi_{j}}{\partial r^{2}}+\frac{2}{r} \frac{\partial \phi_{j}}{\partial r}+\frac{1}{r^{2}} \frac{\partial^{2} \phi_{j}}{\partial \theta^{2}}+\frac{\cos \theta}{r^{2} \sin \theta} \frac{\partial \phi_{j}}{\partial \theta}\right)\right\rangle,
$$

with $1 \leq i, j \leq M$.

\subsubsection{Inversion with constraints}

As the contribution of the radial component of the meridional flow $\left[U_{r}\right]$ to the travel-time measurements is small, it is not feasible to determine this component directly from inverting $\tau$ (Giles 2000). However, the flow components are not independent and they satisfy the steady-state continuity equation. Following Section 3.2.4, we seek the subspace of $\boldsymbol{U}$ that satisfies the mass conservation constraint introduced in matrix form in Equation 3.11 . To reduce the ill-posedness of the problem, we add four boundary constraints so that the flow is confined to the convection zone, following Section 3.2 .4 (see Equations 3.13 and 3.14).

The solution $\boldsymbol{u}$ of the saddle point problem is obtained by solving the matrix equation introduced in Equation 3.19. To choose the regularization parameter $[\alpha]$, the linear system in Equation 3.19 is solved for a wide range of values of $[\alpha]$. To choose the optimal value for the regularization parameter, a trade-off study between bias and error to the estimated solution is needed.

\subsection{Inversion tests with synthetics}

\subsubsection{Synthetic travel times}

In this section, we consider different test cases and perform inversions to tune the linear inversions. To generate synthetic travel times, we construct a single-cell and a double-cell 
meridional flow models following the framework of Liang et al. (2018). Section 3.2.5 briefly explains this framework and how to construct the two synthetic flow models used in the test cases. Synthetic travel times are then generated using a meridional flow model (single- or double-cell) and Born sensitivity kernels and adding a Gaussian noise with zero-mean and the covariance matrix (see Equations 4.1 and 3.7).

To have better comparisons and also not to be dependent to the noise realizations of travel times, we use the same set of noise realizations to generate the synthetic travel times and we consider the mean reconstructed flow for 900 realizations of the noise. Also, the noise level is fixed at a three-year noise level for all the test cases. To choose the optimal regularization parameter, we approximately maintain the same bias for all the test cases. The difference will then be in the propagated errors to the solutions. The propagated error to the solution is obtained by computing the standard deviation of the reconstructed flow over 900 realizations of the noise.

\subsubsection{Tests for mass conservation}

To see the importance of the mass conservation constraint in the current setup, we consider the single-cell flow profile and reconstruct the flow with and without mass conservation. We use the weighted flow vorticity regularization matrix. Note that in the case of nomass-conservation, we remove the related terms to the constraint of mass conservation in Equation 3.19, i.e. $C$ and $\kappa$.

As it is seen in Figure 4.2, the mass conservation has a huge effect on the reconstruction of the flow in both directions, the radial and the colatitudinal components. Using the factor $\beta=10$ in the weighted flow vorticity regularization matrix (see Equation 4.10), Figure 4.2 shows that the mean solution for $U_{r}$ is drastically unstable without mass conservation and has quite large amplitudes. Due to the large cross-talk between $U_{r}$ and $U_{\theta}$ in this case, the mean solution for $U_{\theta}$ is not smooth either. In order to decrease the effect of $U_{r}$ on $U_{\theta}$ for the case of no-mass-conservation, we replace the factor 10 in the weighted flow vorticity regularization matrix by a large factor, e.g. $\beta=10^{5}$, and do the inversions again for the no-mass-conservation case. In this case, $U_{r}$ is almost zero (because it is regularized too much due to the large factor) and the mean reconstructed $U_{\theta}$ is improved and more smoothed. Nevertheless, the mean reconstructed $U_{\theta}$ with mass conservation and using the weighted flow vorticity regularization matrix with $\beta=10$ is much better than the mean reconstructed $U_{\theta}$ with the large factor $\left(\beta=10^{5}\right)$ in the no-mass-conservation case in particular near the poles and at the base of the convection zone.

\subsubsection{Tests for regularization term}

Regularization term plays a crucial role in RLS. To find the optimal regularization matrix $[D]$ in Equation 3.19, we consider the double-cell flow model as input and do inversions using different regularization matrices in the regularization term. Here, we consider the flow vorticity $(\beta=1$ in Equation 4.10), the weighted flow vorticity $(\beta=10$ in Equation 4.10), the flow gradient, and the flow Laplacian regularization matrices.

As Figure 4.3 shows, the weighted flow vorticity regularization matrix gives better mean reconstructed flow for $U_{\theta}$ in particular at high latitudes $\left(\geq \pm 45^{\circ}\right)$ than using the flow vorticity, gradient and Laplacian regularization matrices. Figure 4.4, which displays 

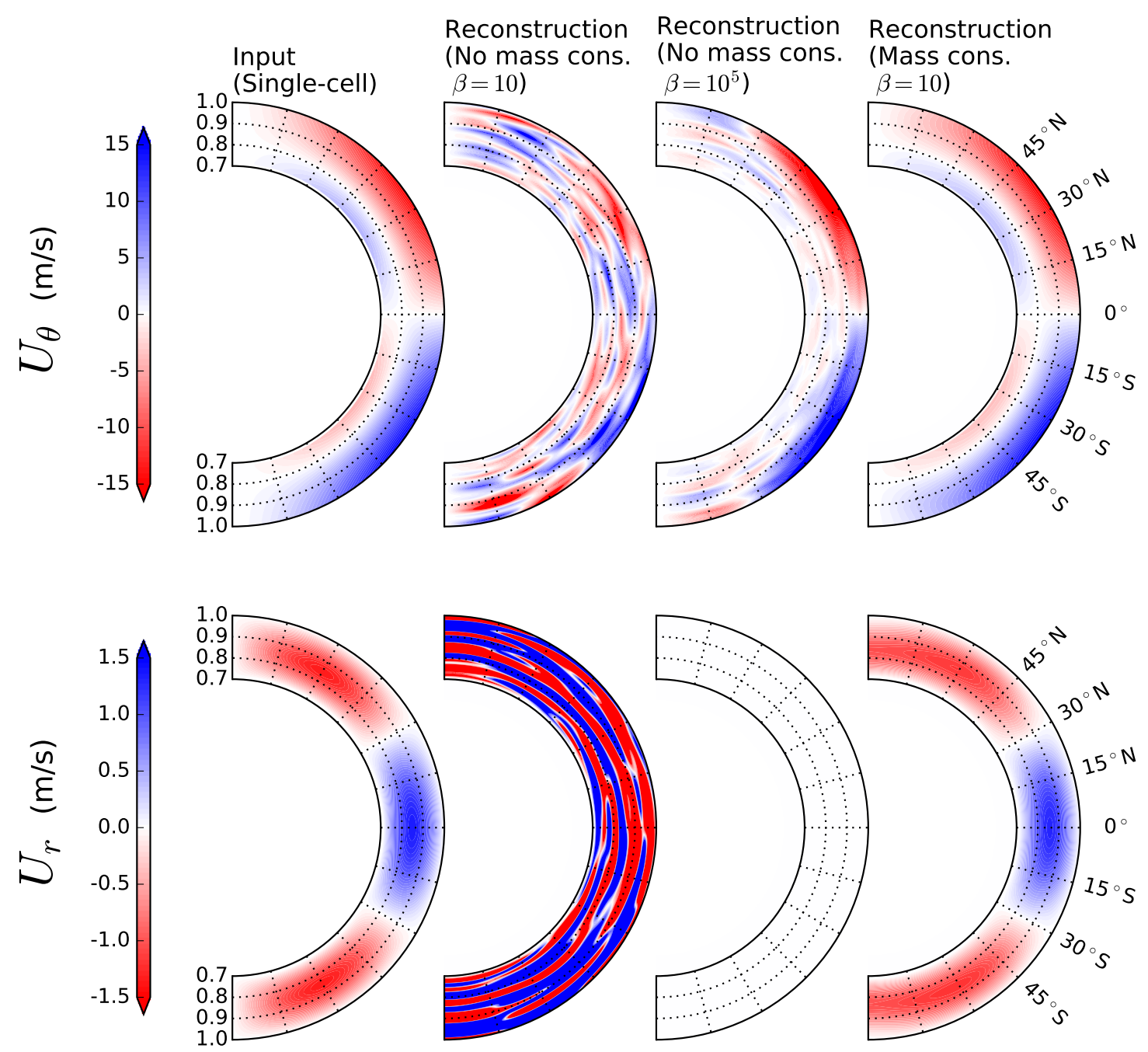

Figure 4.2: (Top panels) $U_{\theta}$ of the single-cell meridional flow input (true solution) and the mean reconstructed flows (900 realizations) for the cases: no-mass-conservation using the weighted flow vorticity regularization matrix with $\beta=10$, no-mass-conservation using the weighted flow vorticity regularization matrix with $\beta=10^{5}$, and with mass conservation using the weighted flow vorticity regularization matrix with $\beta=10$. (Bottom panels) Same as in the top panels, but for $U_{r}$. In the case of the no-mass-conservation using the weighted flow vorticity regularization matrix with $\beta=10, U_{r}$ is varying between $[-41.7,28.3] \mathrm{m} / \mathrm{s}$. 

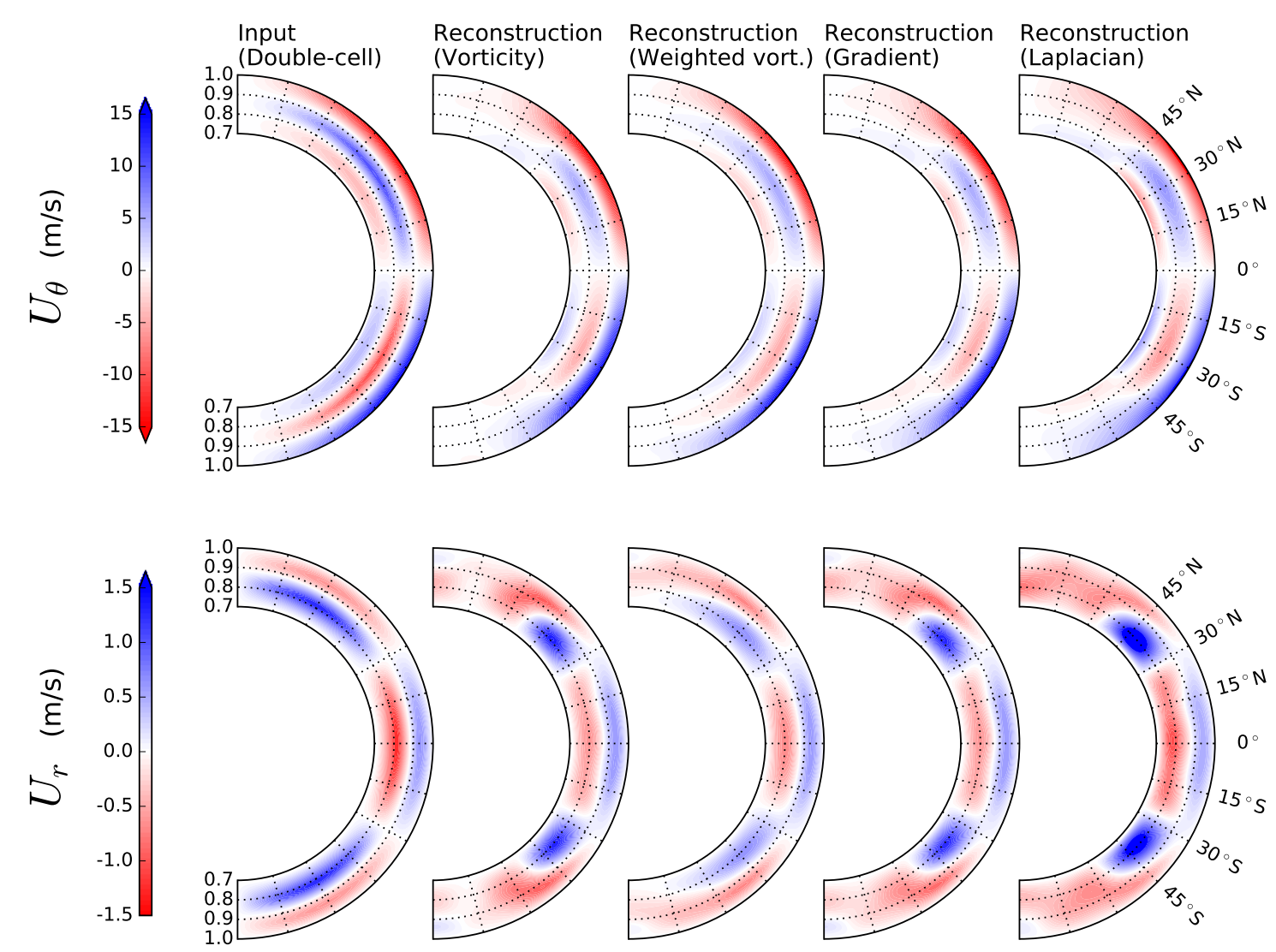

Figure 4.3: (Top panels) $U_{\theta}$ of the double-cell meridional flow input (true solution) and the mean reconstructed flows (900 realizations) using different regularization matrices: the flow vorticity, the weighted flow vorticity, the flow gradient, and the flow Laplacian. (Bottom panels) Same as in the top panels, but for $U_{r}$.

the flow reconstructions for one realization, shows that the factor 10 has a significant effect on the reconstruction of $U_{r}$ and the weighted flow vorticity regularization matrix provides better reconstruction of $U_{r}$ in the whole convection zone than the other regularization matrices. In other words, if the factor 10 is not used in the weighted flow vorticity regularization matrix, $U_{r}$ is under-regularized and has fast flow variations in latitude. We also did inversions using the weighted flow gradient and the weighted flow Laplacian regularization matrices by boosting $U_{r}$ with a factor 10 . The results are quite similar to the results when using the weighted flow vorticity regularization matrix. However, $U_{r}$ of the reconstructed flows for one realization using the weighted flow vorticity and the weighted flow gradient regularization matrices are better than the reconstructed ones using the weighted flow Laplacian regularization matrix.

In addition, with the same bias in the solutions, Figure 4.5 shows that the propagated errors to the solution for $U_{r}$ using the weighted flow vorticity regularization matrix is about two times smaller than to the solutions when using the flow vorticity/gradient regularization matrix. The propagated errors to $U_{r}$ is even larger when using the flow Laplacian regularization matrix. 

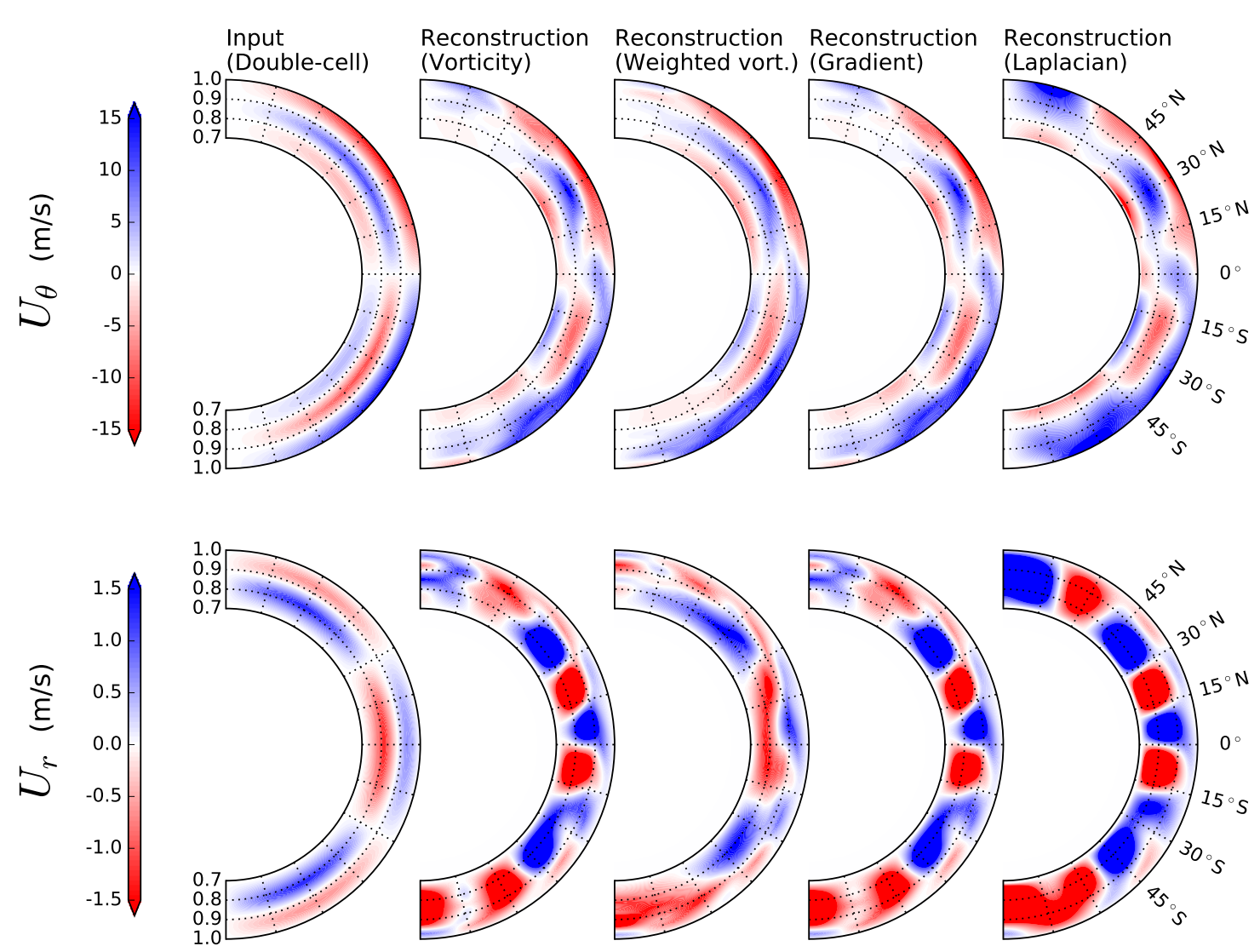

Figure 4.4: (Top panels) $U_{\theta}$ of the double-cell meridional flow input (true solution) and the reconstructed flows (one realization) using different regularization matrices: the flow vorticity, the weighted flow vorticity, the flow gradient, and the flow Laplacian. (Bottom panels) Same as in the top panels, but for $U_{r}$.

\subsubsection{Tests for the boundary constraint}

As solar meridional flow is an axisymmetric flow, imposing $U_{\theta}=0$ along the rotation axis is natural. The radial component of the meridional flow is also zero in the upper boundary, i.e. $U_{r}\left(r=R_{\odot}, \theta\right)=0$ and at the base of the convection zone $U_{r}\left(r=r_{b}, \theta\right)=0$. Using the weighted flow vorticity regularization matrix and the double-cell flow model, Figures 4.6 and 4.7 show that the mean reconstructions of $U_{\theta}$ and $U_{r}$ do not work below $0.8 R_{\odot}$ without the constraint at the base of the convection zone and the values of $U_{r}$ are extremely large and not smooth below this depth. Note that in the case of no constraint at the base of the convection zone, we remove the sub-matrix $S^{1}$, which is responsible for imposing the constraint at the base of the convection zone, from the matrix $S$ in Equation 3.14. This test shows that the constraint at the base of the convection zone is needed to obtain smooth solutions in the deep layers (below $0.8 R_{\odot}$ ).

\subsubsection{Tests for the effect of large separation distances}

Figure 4.8 shows cuts of the mean reconstructed flows using travel times with different ranges of separation distance. The cuts in Figure 4.8 are shown for both the single-cell 

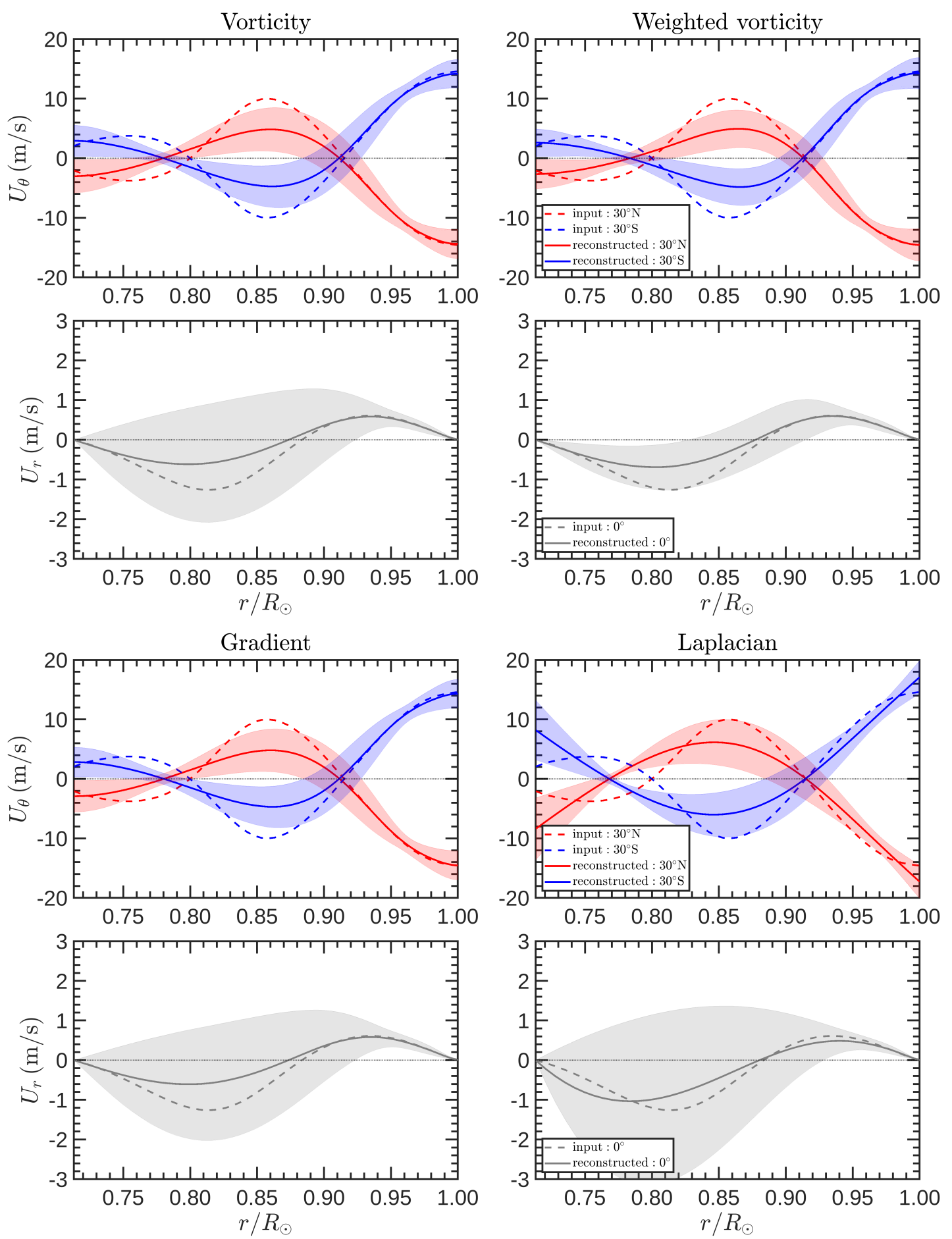

Figure 4.5: Radial cuts of $U_{\theta}$ at $\pm 30^{\circ}$ latitude and $U_{r}$ at the equator showing the mean reconstructed flow and its standard deviation (solid lines and shading), compared to the input double-cell flow profile (dashed curve) using the flow vorticity (top left), the weighted flow vorticity (top right), the flow gradient (bottom left), and the flow Laplacian (bottom right) regularization matrices. 

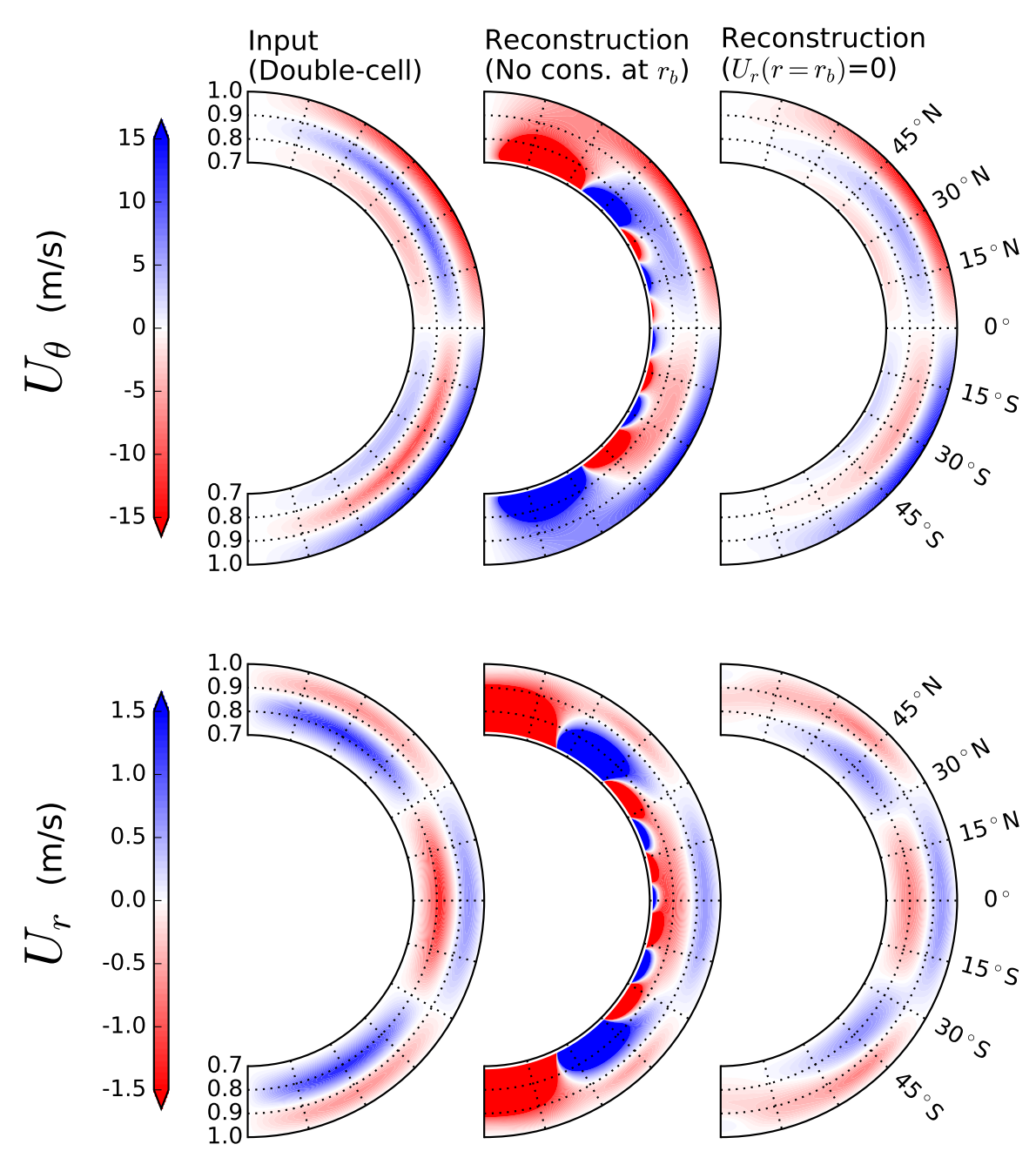

Figure 4.6: (Top panels) $U_{\theta}$ of the double-cell meridional flow input (true solution) and the mean reconstructed flows (900 realizations) without and with the constraint $U_{r}(r=$ $\left.r_{b}\right)=0$ using the weighted flow vorticity regularization matrix. (Bottom panels) Same as in the top panels, but for $U_{r}$.

and double-cell meridional flow inputs. The results of the cases $\Delta \leq 30^{\circ}$ and $\Delta \leq 42^{\circ}$ are not distinguishable. In other words, the results show that the travel times with separation distances $\Delta>30^{\circ}$ do not contribute significantly to the inversions.

In order to assess the importance of the travel times with large separation distances $\left(\Delta>30^{\circ}\right)$, we artificially decrease the noise of these travel times by a factor 100 . The mean reconstruction improves mainly near the middle of the convection zone with a reduction in bias by a factor 1.4 for the single-cell flow model and a reduction in bias by a factor 1.5 for the double-cell flow model. The mean reconstruction also improves with a reduction in noise by a factor 1.2. Hence, we conclude that the large-distance travel times $\left(\Delta>30^{\circ}\right)$ do not contribute significantly to the inversions. Firstly, because they have large noise and secondly, because the constraint of mass conservation has significant contribution to the determination of the deep meridional flow. 

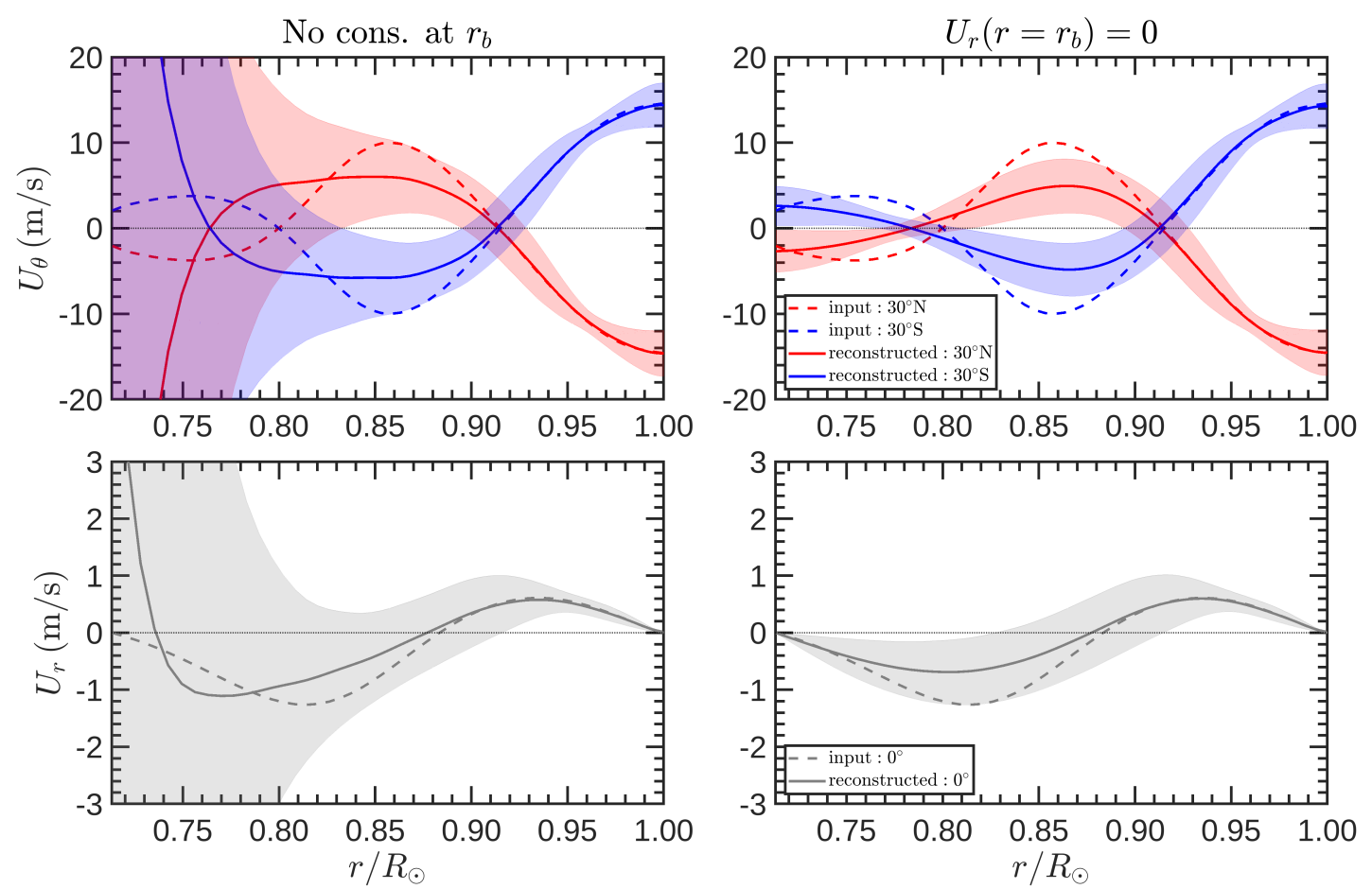

Figure 4.7: (Top panels) Radial cuts of $U_{\theta}$ at $\pm 30^{\circ}$ latitude and (bottom panels) $U_{r}$ at the equator showing the mean reconstructed flow and its standard deviation (solid lines and shading), compared to the input double-cell flow profile (dashed curve) without and with the constraint $U_{r}\left(r=r_{b}\right)=0$ using the weighted flow vorticity regularization matrix.

\subsection{Conclusion}

In this chapter, we tuned and validated the helioseismic inversions to infer the solar meridional flow using time-distance helioseismology. We used the first-order Born sensitivity kernels and realistic noise to generate synthetic travel times. Different test cases were considered related to different parts of the inversion setup. We find that the mass conservation constraint is necessary to reconstruct the radial component of the meridional flow $\left[U_{r}\right]$. This constraint also improves the colatitudinal component of the reconstructed flow $\left[U_{\theta}\right]$. The weighted flow vorticity regularization matrix is superior to other choices of the regularization matrix. It gives the best reconstructions of $U_{\theta}$ at high latitudes $\left(\geq \pm 45^{\circ}\right)$ and of $U_{r}$ in the whole convection zone. With the same bias in the solution, the weighted flow vorticity regularization matrix provides smaller errors (approximately two times) to the solution for $U_{r}$ in comparison to the flow vorticity and gradient regularization matrices.

Implementing the constraint $U_{r}\left(r=r_{b}\right)=0$, where $r_{b}$ denotes the radius of the base of the convection zone, is necessary to reconstruct the flow below $0.8 R_{\odot}$. The contribution of the travel times with large separation distances $\left(\Delta>30^{\circ}\right)$ to the inversions is not significant. One reason is that these travel times have large noise. Another reason is that the constraint of mass conservation has significant contribution to the determination of the deep meridional flow. 

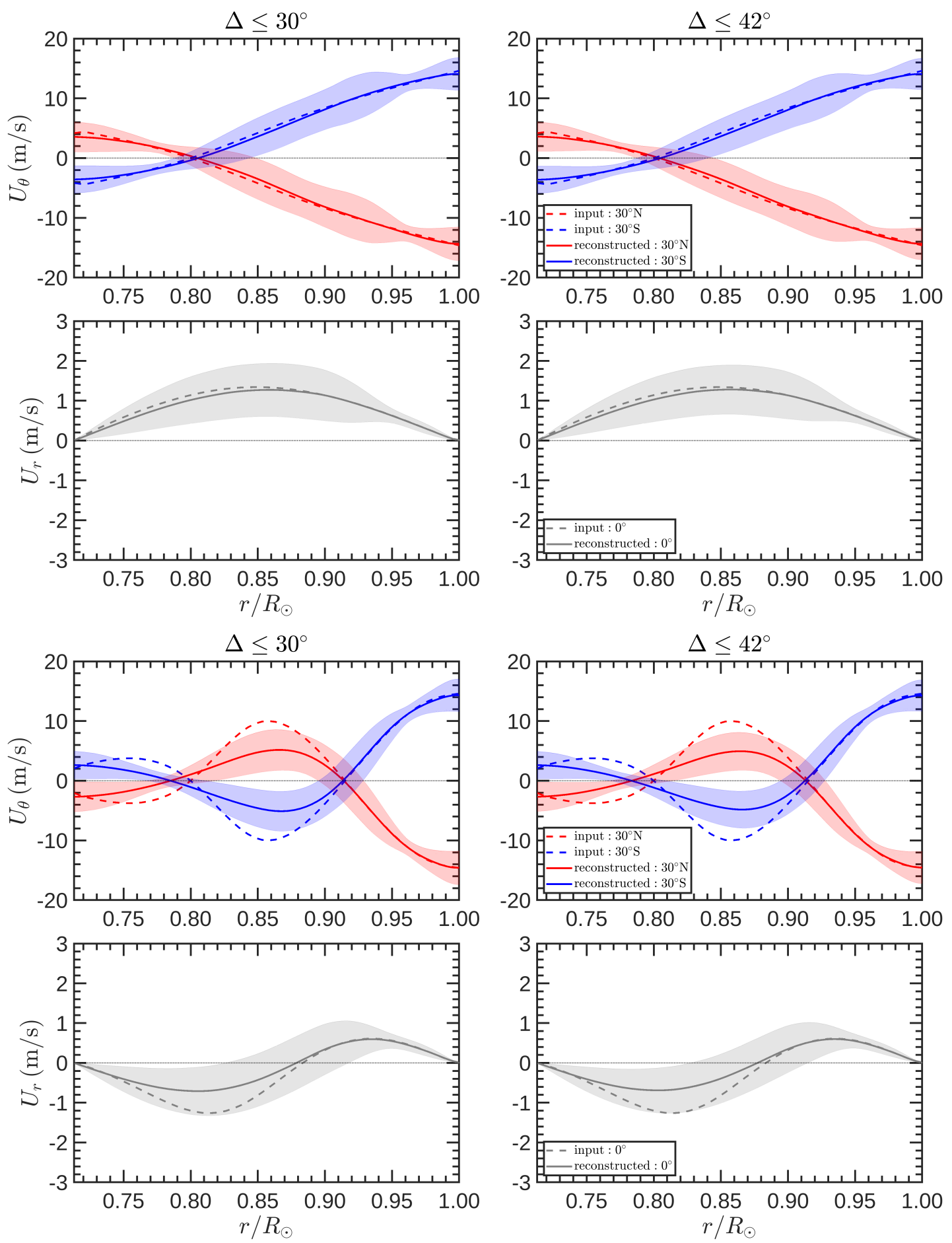

Figure 4.8: Radial cuts of $U_{\theta}$ at $\pm 30^{\circ}$ latitude and $U_{r}$ at the equator showing the mean reconstructed flow and its standard deviation (solid lines and shading), compared to the input single-cell flow profile (dashed curve) using travel times with separation distances $\Delta \leq 30^{\circ}$ (top left) and $\Delta \leq 42^{\circ}$ (top right) using the weighted flow vorticity regularization matrix for the single-cell meridional flow input. (Bottom) Same as in the top, but for the double-cell meridional flow input. 



\section{Discussion}

In this section, I compare the spatial resolutions of inversions for flows for different choices of helioseismic observables at fixed noise level. In addition to the standard techniques used in the thesis so far, I also consider full-waveform inversions as a new approach. I then provide a summary of the conclusions obtained in this thesis, and I present a few suggestions for future work.

\subsection{Helioseismic observables and forward modeling}

Interpreting the helioseismic data and performing helioseismic inversions are challenging since there is a very large amount of data and there is high level of noise in the data. Therefore, averaging helioseismic data is necessary to increase the signal-to-noise ratio. This leads to intermediate data products obtained from the cross-correlation of the oscillation signal (e.g. the Doppler velocity) between pairs of points on the solar surface. Regarding the inverse problem, different types of input data, also known as observables, can thus be used in helioseismic inversions.

Different choices of helioseismic observables can lead to different systematic errors and different signal-to-noise ratios. These differences in the observables might lead to inversion solutions with different spatial resolutions and propagated errors to the solution or even contradictory solutions. In this section, ongoing work corresponding to different helioseismic observables is discussed. The focus is on comparing the averaging kernels of inversions for different choices of helioseismic observables at fixed noise level for recovering flows at a given $3 \mathrm{D}$ location in the solar interior.

As it was mentioned in Section 1.2, the cross-covariance function is a solar seismogram and it contains information about travel times and amplitudes of the wave packets traveling between any two surface locations. We define the cross-covariance function in the frequency domain as

$$
C_{i}(\omega)=\frac{2 \pi}{T} \psi^{*}\left(\boldsymbol{r}_{i}, \omega\right) \psi\left(\boldsymbol{r}_{i}^{\prime}, \omega\right),
$$

where $\psi$ denotes the observed signal, $\omega$ is the angular frequency, $\boldsymbol{r}_{i}=\left(R_{\odot}, \theta_{i}\right)$ and $\boldsymbol{r}_{i}^{\prime}=$ $\left(R_{\odot}, \theta_{i}^{\prime}\right)$ are two locations on the solar surface, and $T$ is the duration of observation.

As the forward problem introduced in Equation 1.2 in Section 1.3, in time-distance helioseismology one seeks to relate the helioseismic surface measurements to the internal physical properties. In this study, the measurements of the cross-covariance function in the frequency domain (full-waveform observable) are related to the subsurface meridional flow as

$$
d_{i}(\omega)=\iint_{A} \boldsymbol{K}_{i}(\omega, \boldsymbol{r}) \cdot \boldsymbol{U}(\boldsymbol{r}) \mathrm{d} \boldsymbol{r}+n_{i}(\omega),
$$


where $\boldsymbol{U}(\boldsymbol{r})=\left(U_{r}(\boldsymbol{r}), U_{\theta}(\boldsymbol{r})\right)$ denotes the vector flow, $d_{i}(\omega)$ is a set of cross-covariance measurements with the corresponding noise $n_{i}$, and $\boldsymbol{K}_{i}=\left(K_{i}^{r}, K_{i}^{\theta}\right)$ where $K_{i}^{r}$ and $K_{i}^{\theta}$ are the sensitivity kernels in the radial and colatitudinal directions. The kernels in Equation 5.2 are longitudinally averaged. The position vector $\boldsymbol{r}=(r, \theta)$ belongs to the half-disk of radius $R_{\odot}$, denoted by $A$.
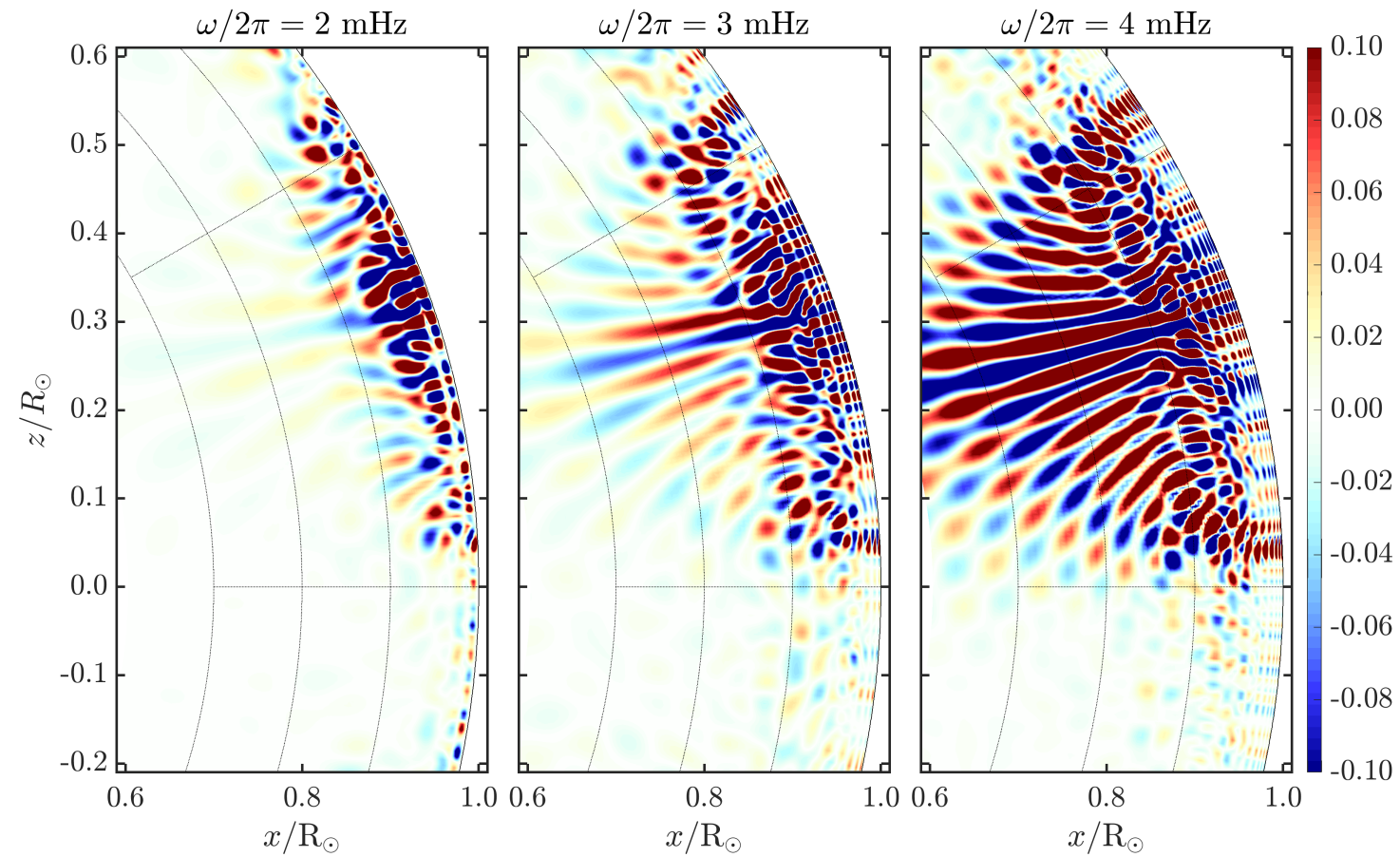

Figure 5.1: Cross-covariance sensitivity kernels $K^{\theta}(\omega, \boldsymbol{r})$ for $U_{\theta}$ centered at three different frequencies 2, 3, and $4 \mathrm{mHz}$. The kernels correspond to a pair of points located at the latitudes $3^{\circ}$ and $33^{\circ}$ on the solar surface. Only imaginary parts of the kernels are shown and spherical harmonic degrees up to $l=300$ are used to compute the kernels. For illustrative purposes, the values of the kernels in each panel are scaled by the sound speed, normalized by the maximum absolute value and saturated. The kernels are computed following the framework of Fournier et al. (2018).

Here, "cross-covariance measurements" denoted by $d_{i}(\omega)$, are the cross-covariance perturbations due to the meridional flow with respect to a reference cross-covariance $C_{\text {ref }}$. As Equation 5.2 indicates, there are cross-covariance sensitivity kernels at each frequency. Figure 5.1 shows the cross-covariance sensitivity kernels (imaginary parts) for $U_{\theta}$ corresponding to a pair of points on the solar surface at three different frequencies. The cross-covariance kernels show different levels of sensitivity in the solar interior for different frequencies and every single frequency contains certain information about the waves propagating in the solar interior.

Following the approaches presented in Gizon and Birch (2002) and Nagashima et al. (2017), we extract travel-time and amplitude measurements from the cross-covariance. We define the "travel-time measurement" as the time lag and "amplitude measurement" as the relative cross-covariance amplitude that minimize $\delta C=C-C_{\text {ref }}$ (in the sense of least squares) isolating the first-skip branch of the cross-covariance in the time domain. 
Thus, forward problem stated in Equation 5.2 for travel-time and amplitude measurements shrinks to

$$
d_{i}=\iint_{A} \boldsymbol{K}_{i}(\boldsymbol{r}) \cdot \boldsymbol{U}(\boldsymbol{r}) \mathrm{d} \boldsymbol{r}+n_{i},
$$

where $d_{i}$ denotes either travel-time or amplitude measurements. Therefore, travel-time and amplitude measurements only depend on the observational points since they are frequency-averaged quantities. Figure 5.2 shows the sensitivity kernels of the different helioseismic observables for $U_{\theta}$ corresponding to a pair of points located on the solar surface.
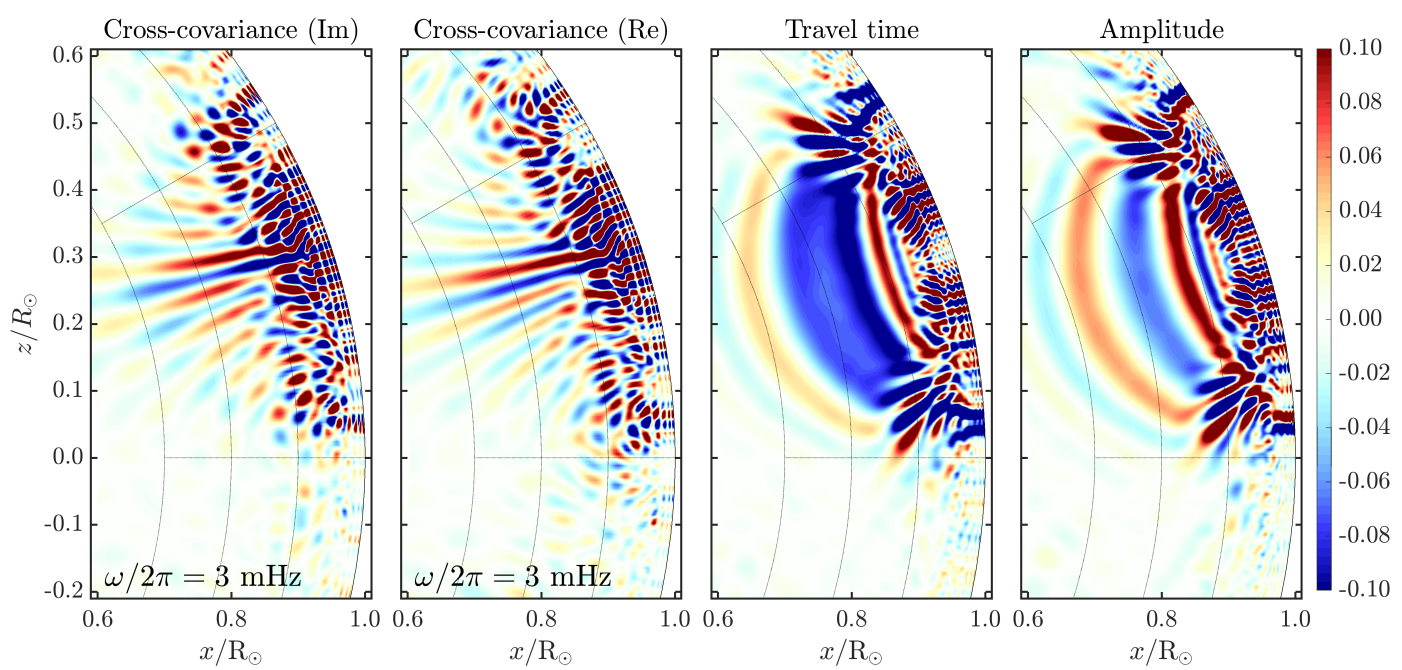

Figure 5.2: Sensitivity kernels of the cross-covariance (imaginary and real parts at $3 \mathrm{mHz}$ ), travel-time and amplitude measurements for $U_{\theta}$. The kernels correspond to a pair of points located at the latitudes $3^{\circ}$ and $33^{\circ}$ on the solar surface. Spherical harmonic degrees up to $l=300$ are used to compute the kernels. For illustrative purposes, the values of the kernels in each panel are scaled by the sound speed, normalized by the maximum absolute value and saturated. The kernels are computed following the framework of Fournier et al. (2018).

So far different helioseismic observables and their corresponding forward problems are introduced. We seek to probe flows down to the depth of the convection zone, so it is important to compare the spatial resolutions of inversions for flows for different choices of helioseismic observables. For this aim, we consider a 2D problem. Figure 5.3 shows the 2D setup of the problem and some sample ray paths for different separation distances. The positions of the observational surface points are chosen on uniform grid within the latitudes $\pm 60^{\circ}$ from the equator, sampled every $3^{\circ}$. All possible combinations of the observational points were computed from this grid. Those pairs with separation distance greater than $\Delta=42^{\circ}$ were removed, as these distances present difficulties for observational interpretation (Liang et al. 2017). The point-to-point sensitivity kernels are then computed for these observational pairs of points. Note that sensitivity kernels are also computed for different frequencies in the case of cross-covariance. Frequencies between 


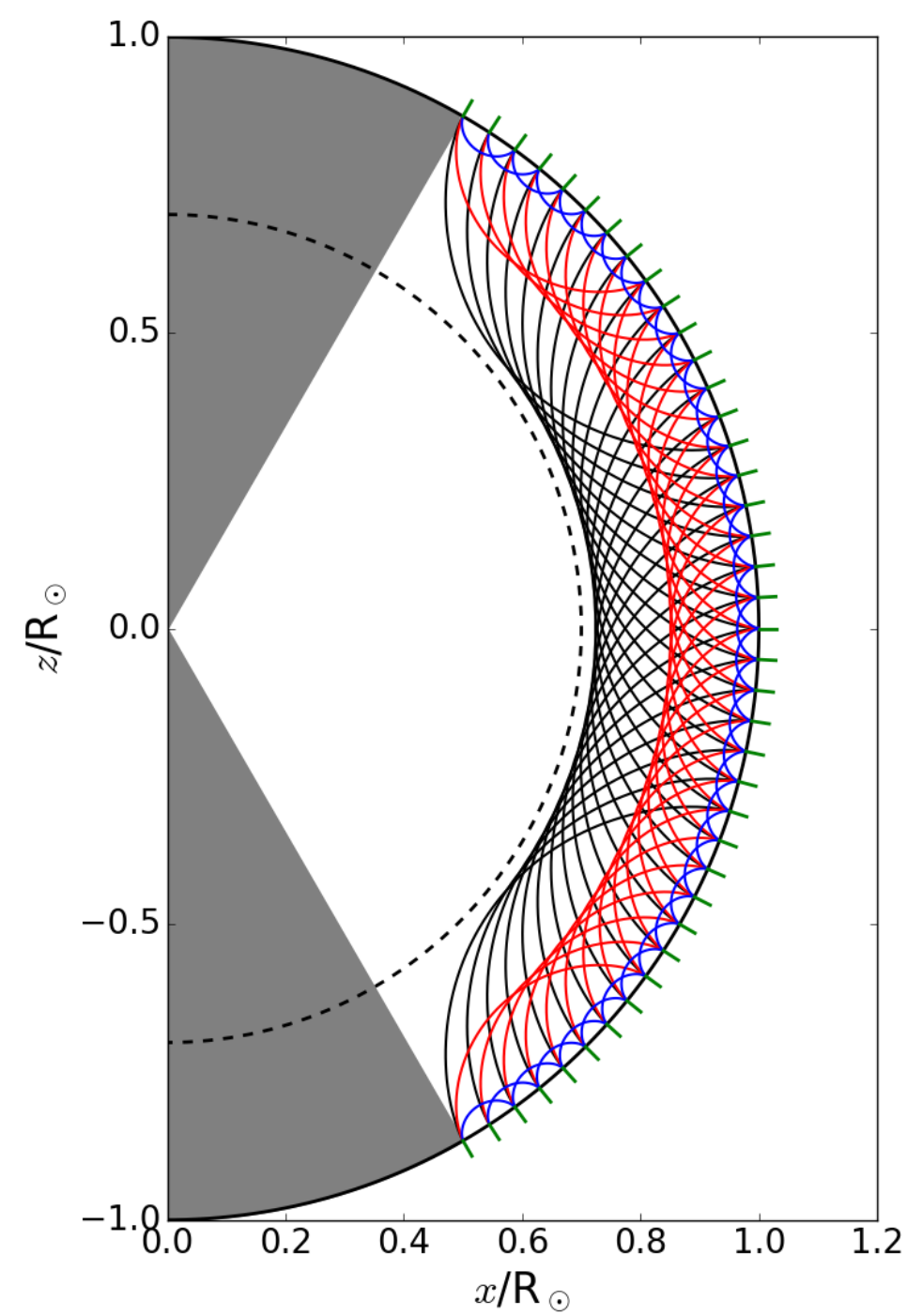

Figure 5.3: The pairs of points (green ticks) chosen for this study. The ray paths between these points are shown for pairs separated by $6^{\circ}$ (blue), $24^{\circ}$ (red) and $42^{\circ}$ (black). In order to remain consistent with observational capabilities we have neglected observational points located further than $60^{\circ}$ from the equator. The dashed line indicates the base of the convection zone.

[2-4] $\mathrm{mHz}$ are considered for the cross-covariance measurements. The kernels are computed following the framework of Fournier et al. (2018).

\subsection{Comparison of inversion strategies}

To compare different inversion strategies by considering different choices of helioseismic observables, we express the flow components as linear combinations of basis functions as introduced in Section 3.2.2. We discretize the problem following the same approach explained in Section 3.2.2 except that we use piecewise constant basis functions in both the radial and horizontal directions in this study. 
As it is explained in Section 1.3, inverse problems in helioseismology are mostly solved linearly that means we seek to find the solution vector of flow coefficients $\hat{\boldsymbol{u}}$ as linear combination of the input data vector $\boldsymbol{d}$ as (see Equation 1.10)

$$
\hat{\boldsymbol{u}}=\mathcal{W} \boldsymbol{d}
$$

where $\mathcal{W}$ is the weight matrix to be determined. Note that the input data vector $\boldsymbol{d}$ in Equation 5.4 can be the data set of any types of observables such as cross-covariance measurements, travel times or amplitudes. To find the weight matrix $\mathcal{W}$ (see Equation 1.12 , we seek to minimize the RLS cost function (see Equation 1.11) with respect to the vector of flow coefficients $\boldsymbol{u}$

$$
\hat{\boldsymbol{u}}=\operatorname{argmin}_{\boldsymbol{u}}\left\{\left\|\Lambda^{-1 / 2}(K \boldsymbol{u}-\boldsymbol{d})\right\|^{2}+\alpha\|\boldsymbol{u}\|^{2}\right\},
$$

where $\|\cdot\|$ denotes the discrete $L^{2}$ norm, $K$ is the matrix containing the kernel coefficients (see Equation 1.9), $\Lambda$ is the noise covariance matrix of the measurements, and $\alpha$ is a regularization parameter.

To investigate the intrinsic properties of the forward models of the different observables, we utilize the singular value decomposition (SVD) (e.g. Hansen 2010) which provides information about the intrinsic properties of a matrix. The SVD of the $N \times M$ matrix $\Lambda^{-1 / 2} K$ of rank $R$ is a factorization of $\Lambda^{-1 / 2} K$ into the product of three matrices as:

$$
\Lambda^{-1 / 2} K=\mathcal{U} \Sigma \mathcal{V}^{H}
$$

where the superscript $H$ denotes the Hermitian conjugate, $\mathcal{U}$ and $\mathcal{V}$ are isometric matrices of sizes $N \times N$ and $M \times M$, respectively (i.e. $\mathcal{U}^{H} \mathcal{U}=\mathbb{I}_{N}$ and $\mathcal{V}^{H} \mathcal{V}=\mathbb{I}_{M}$ ), and $\Sigma$ is an $N \times M$ rectangular diagonal matrix. The diagonal entries $\sigma_{k}$ of $\Sigma$ for $k=1, \ldots, R$ are known as the singular values of $\Lambda^{-1 / 2} K$, and we assume that they are sorted by size:

$$
\sigma_{1} \geq \sigma_{2} \geq \ldots \geq \sigma_{R}>0
$$

The ill-posedness of the inverse problem is reflected in the spectrum of the singular values of the matrix $\Lambda^{-1 / 2} K$. As Figure 5.4 shows, the singular values for crosscovariance measurements decay slower than the singular values for travel-time/amplitude measurements. The faster the singular values decay, the more ill-posed the problem is (e.g. Christensen-Dalsgaard et al. 1993). The slower decay of the cross-covariance singular values is because more information is present in the cross-covariance than in travel times/amplitudes.

Inverse problem is a trade-off between bias and variance. These quantities can be extracted from the difference between the reconstructed and exact flow coefficients as

$$
\begin{aligned}
& \mathbb{E}\left[\|\hat{\boldsymbol{u}}-\boldsymbol{u}\|^{2}\right]=\mathbb{E}\left[\|\boldsymbol{W} \boldsymbol{d}-\boldsymbol{u}\|^{2}\right] \\
& =\|(\mathcal{K}-\mathbb{I}) \boldsymbol{u}\|^{2}+\|\mathcal{W}\|^{2},
\end{aligned}
$$

where $\mathbb{E}$ denotes the expectation value of a stochastic variable and

$$
\mathcal{K}=\mathcal{W} K,
$$




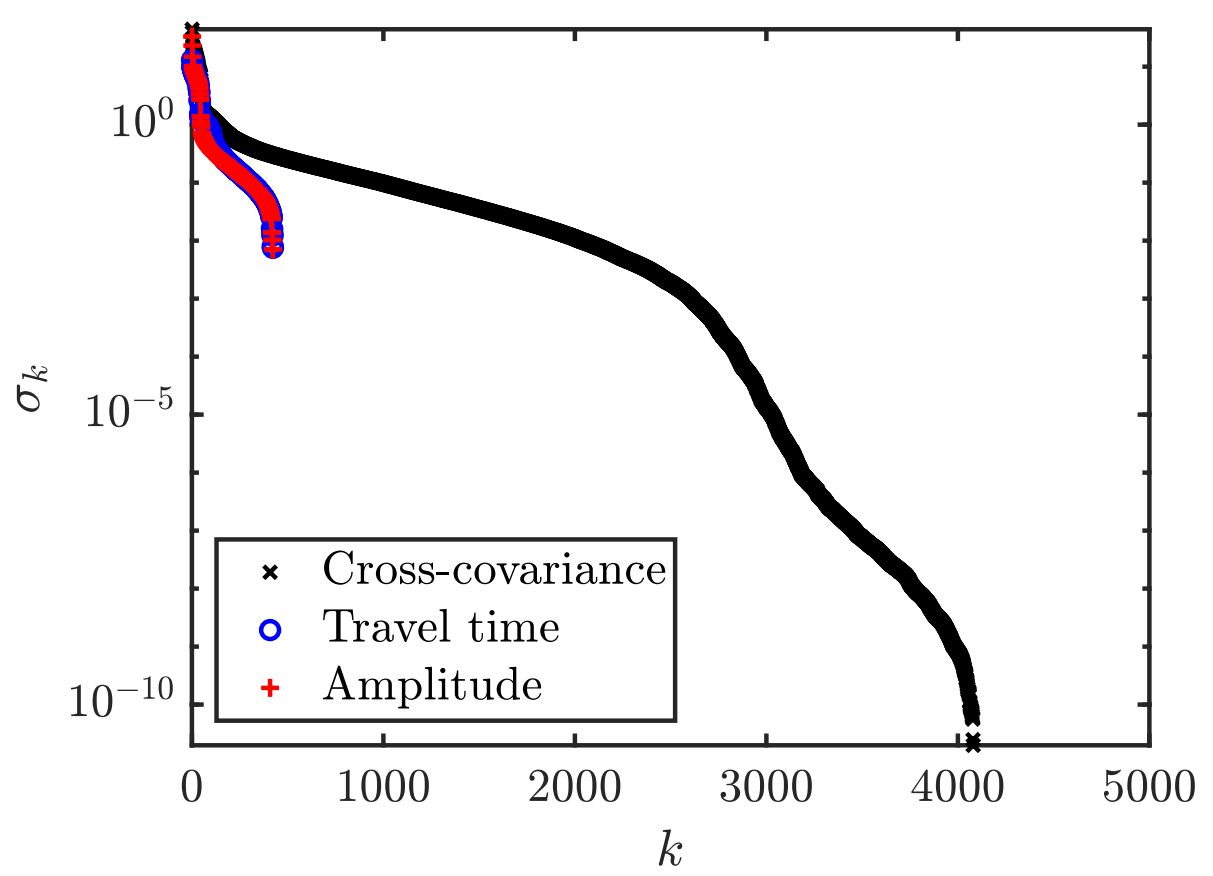

Figure 5.4: Spectrum of the singular values $\sigma_{k}$ for the cross-covariance, travel-time and amplitude measurements. More singular values are available for the cross-covariance since there are more measurements in the cross-covariance than travel times/amplitudes.

is the averaging kernel. The matrix $\mathcal{K}$ in Equation 5.9 contains the averaging kernels for $U_{\theta}, U_{r}$, and the corresponding cross-talk averaging kernels.

Averaging kernel demonstrates the resolution of the inversion at each chosen target position $\boldsymbol{r}_{0}=\left(r_{0}, \theta_{0}\right)$. The first term in Equation 5.8 defines how far the averaging kernel is far from the identity, i.e. the bias of the estimator. The second term in Equation 5.8 corresponds to the variance of the estimator, i.e. the propagated error to the estimated solution in Equation 5.4.

Example averaging kernels of inversions for $U_{\theta}$ at a chosen target location for the three choices of observables are shown in Figure 5.5. With the same noise level in the solution (propagated error), the averaging kernel is more localized when using the cross-covariance function in the frequency domain (FWI), rather than travel times or amplitudes as input data. This means that the FWI provides solutions with better spatial resolutions and thus less bias at fixed noise level. This more localized averaging kernel of FWI is due to the more available information in the cross-covariance data set than in travel times/amplitudes. Even though each single travel-time/amplitude measurement has a higher signal-to-noise ratio than a single cross-covariance measurement, the subspace of linear combinations of the travel-time/amplitude data set is smaller than FWI in the reconstruction. This point can also be seen from the spectrum of the singular values of the observables as shown in Figure 5.4 as the decay rate of the singular values is way slower for cross-covariance than travel time or amplitude. Radial cuts through averaging kernels of FWI at different chosen target locations are shown in Figure 5.6. Comparing the full width at half maximum of the averaging kernels in the radial direction at each target location to the local wavelength $\lambda$, the spatial resolution of FWI is close to $\lambda / 2$ which is the diffraction limit of acoustic waves. 

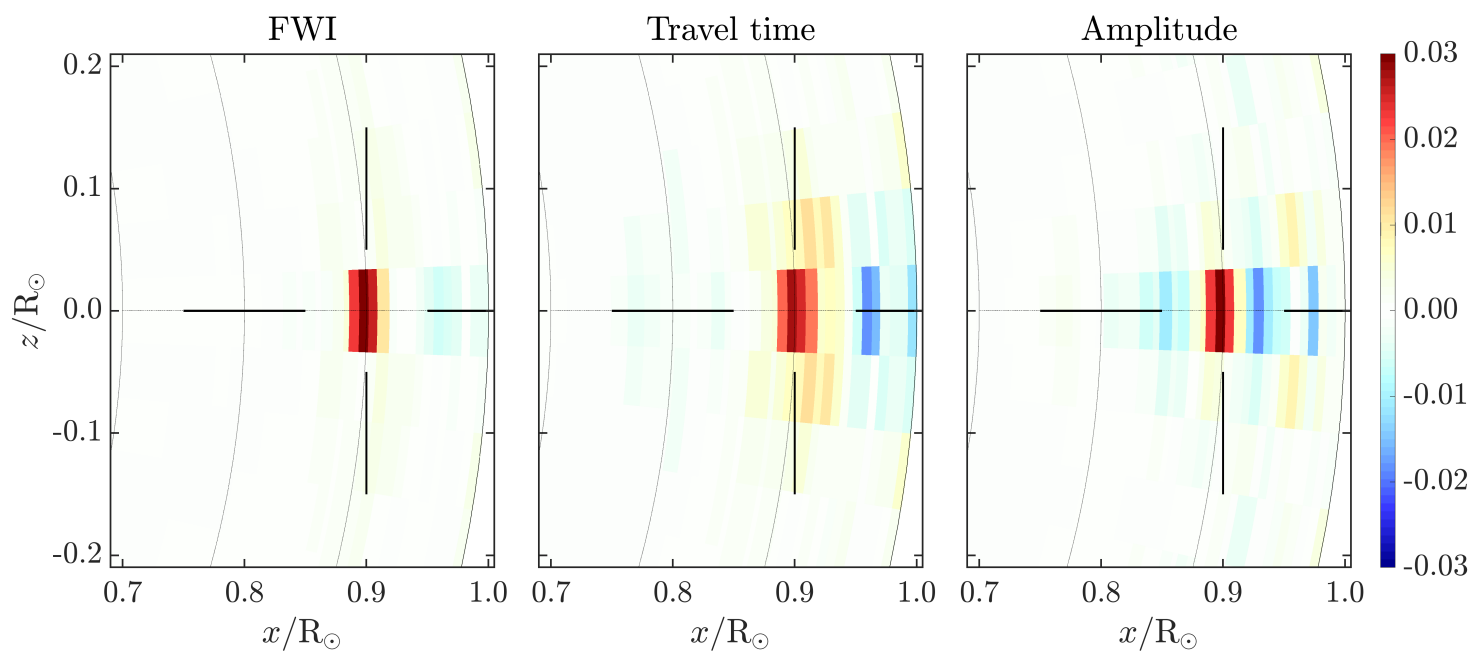

Figure 5.5: Averaging kernels of FWI (left panel), travel-time (middle panel) and amplitude (right panel) inversions for $U_{\theta}$ considering $T=4$ years. The crosshairs (solid lines) indicate the target location at $r_{0} / R_{\odot}=0.9$ at the solar equator. The propagated error to the solutions at this chosen target is about $1.5 \mathrm{~m} / \mathrm{s}$ for the three observables. Frequencies between [2-4] $\mathrm{mHz}$ are considered for the cross-covariance measurements.

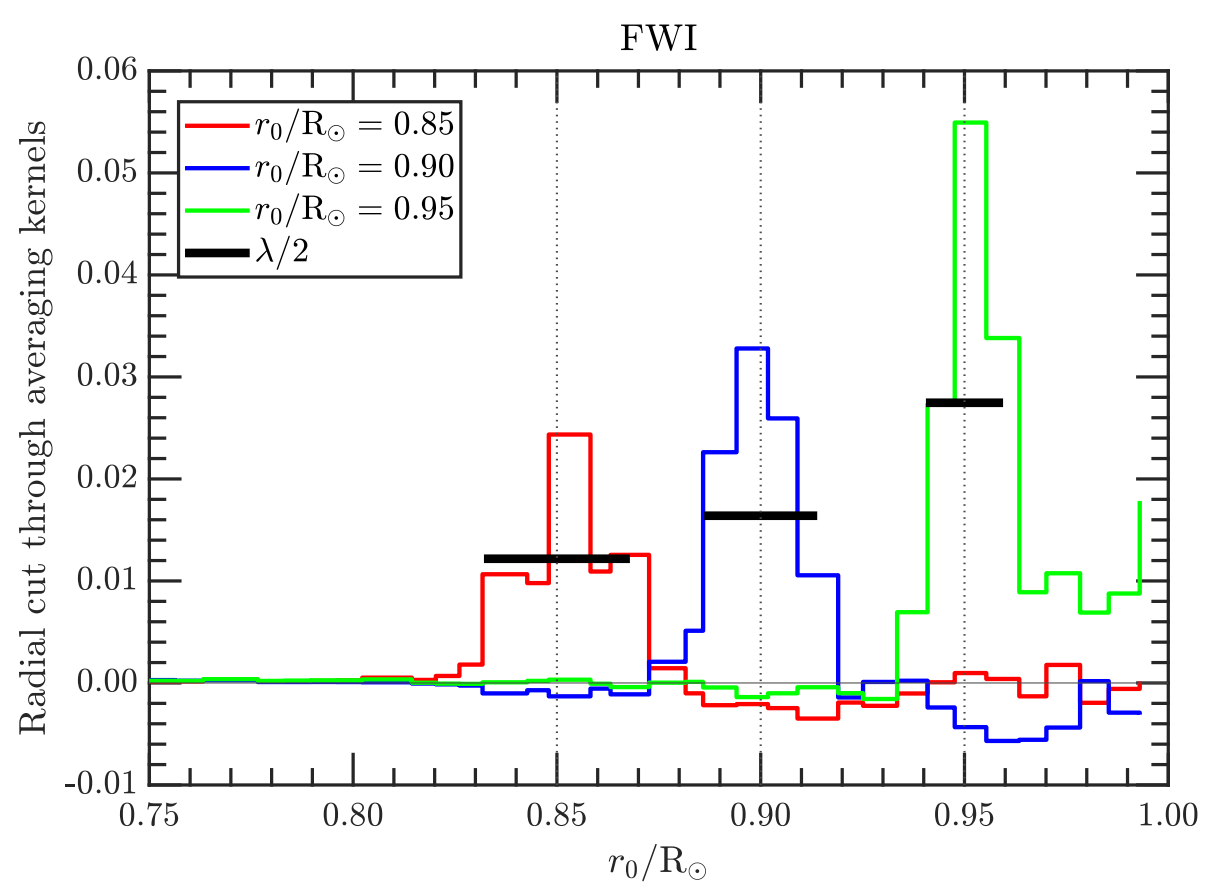

Figure 5.6: Radial cuts through averaging kernels of FWI for $U_{\theta}$ at three chosen target locations considering $T=4$ years (see the left panel of Figure 5.5 targeting at $r_{0} / R_{\odot}=$ 0.9). The dots indicate the depths of the target points at $r_{0} / R_{\odot}=[0.85,0.9,0.95]$ at the solar equator. The thick lines plotted at the half maximums of the averaging kernels are of length $\lambda / 2$ where $\lambda$ denotes the local wavelength at $3 \mathrm{mHz}$. The propagated errors to the solutions at $r_{0} / R_{\odot}=[0.85,0.9,0.95]$ are about $2,1.5$, and $1 \mathrm{~m} / \mathrm{s}$, respectively. 


\subsection{Conclusions}

By performing helioseismic inversions of wave travel times, the main objective of this dissertation was to contribute to a better understanding of the solar meridional flow which is a crucial ingredient to understand the dynamics in the Sun. Besides, the helioseismic inversions are tuned and validated with synthetic travel times using different flow profiles. In the following, the main conclusions obtained in this thesis regarding the inversions for the solar meridional flow are presented:

- By using synthetic travel times, we find that mass conservation is a necessary constraint to reconstruct the radial component of the meridional flow. Using the regularized least squares method, the weighted flow vorticity in the regularization term gives the best reconstructions of the meridional flow in both the radial and horizontal directions compared to the vorticity, gradient and Laplacian of the flow. The contribution of the travel times with separation distances $\Delta>30^{\circ}$ to the inversions is not significant. This is due to the large noise of the travel times with separation distances $\Delta>30^{\circ}$ and also significant role of the mass conservation constraint for determination of the deep meridional flow,

- By inverting the helioseismic travel times over the solar cycles 23 and 24 (19962019), the inferred solar meridional flow is varying through the whole convection zone. For both cycles, the inferred solar meridional flow has a single-cell structure in each hemisphere: poleward at the surface and equatorward at the base of the convection zone. At the base of the convection zone, the colatitudinal component of the inferred meridional flow is approximated with a function given by $U_{\theta}=U_{b} \sin 2 \theta$ with $U_{b}=4.8 \pm 1.0 \mathrm{~m} / \mathrm{s}$ for cycle 23 and $U_{b}=3.6 \pm 1.0 \mathrm{~m} / \mathrm{s}$ for cycle 24 . The sign of the flow flips at a depth of about 0.79 solar radius. Besides, confidence in the results is provided by the agreement between SOHO/MDI and GONG data during the period $2001-2011$ and also by finding a single-cell solution for the meridional flow for each one of the four seasons. Under a flux-transport dynamo model, the inferred single-cell meridional flow is able to explain the equatorial migration of sunspots in each hemisphere.

Another objective of this dissertation was to analyze other possibilities than using travel times as observables in time-distance helioseismology. The main conclusions obtained in this thesis regarding the application of different observables in time-distance helioseismology are presented:

- Considering sound-speed perturbations to a uniform background medium, we extracted the travel-time and amplitude measurements from the deep-focusing crosscovariance functions and derived the spatial sensitivity kernels under the first Born approximation. The spatial sensitivity of deep-focusing travel-time measurements is zero at the target location and negative in a surrounding region with diameter approximately equal to the width of the first Fresnel zone, $L_{F}$. However, the spatial sensitivity of amplitude measurements peaks at the target location and is negative in a region with diameter approximately equal to $0.7 L_{F}$. Hence, the deep-focusing sensitivity kernels for amplitudes are more localized than the deep-focusing sensitivity kernels for travel-time measurements. This leads to a higher signal-to-noise 
ratio for deep-focusing amplitude measurements than for deep-focusing travel-time measurements for small-scale perturbations in sound speed. The conclusion is that amplitude measurements are useful in addition to the travel times in local helioseismology as they provide additional information about the solar subsurface structure from travel times.

- With the same noise level in the reconstructions, averaging kernels for flows at any chosen target location in the solar interior are more localized when using the cross-covariance function in the frequency domain (FWI) rather than travel times or cross-covariance amplitudes as input data. The spatial resolution of FWI is close to half the local wavelength, $\lambda / 2$. The conclusion is that the full-waveform approach is promising for future helioseismic studies.

A very first suggestion for future work would be the confirmation of the RLS inversions for the meridional flow using other types of helioseismic inverse methods, e.g. SOLA (e.g. Pijpers and Thompson 1994, Jackiewicz et al.|2012, Böning et al.|2017, Korda et al. 2019).

Current inversions can also be improved. For instance, a penalty can be placed on the cross-talk between the different solar properties we are inverting for, such as the solar flow components and the sound-speed perturbations (Švanda et al. 2011). Moreover, the effects of imperfect kernels on the reconstructed flows can be studied in order to improve the forward modeling.

Regarding the helioseismic approach and methods, full-waveform inversion can be applied for the inferences of the solar meridional flow. The advantage of full-waveform inversion is that independent information can be used from different frequencies. However, systematics in the input data might be less known than the ones in travel times. 



\section{Bibliography}

Appourchaux, T., Belkacem, K., Broomhall, A. M., Chaplin, W. J., Gough, D. O., Houdek, G., Provost, J., Baudin, F., Boumier, P., Elsworth, Y., García, R. A., Andersen, B. N., Finsterle, W., Fröhlich, C., Gabriel, A., Grec, G., Jiménez, A., Kosovichev, A., Sekii, T., Toutain, T., Turck-Chièze, S., 2010, The quest for the solar g modes, A\&A Rev., 18, 197-277, 0910.0848

Backus, G., Gilbert, F., 1968, The Resolving Power of Gross Earth Data, Geophysical Journal, 16, 169-205

Basu, S., Antia, H. M., 2010, Characteristics of Solar Meridional Flows during Solar Cycle 23, ApJ, 717, 488-495, 1005.3031

Beck, J. G., Giles, P., 2005, Helioseismic Determination of the Solar Rotation Axis, ApJ, 621, L153-L156

Beck, J. G., Gizon, L., Duvall, T. L., J., 2002, A New Component of Solar Dynamics: North-South Diverging Flows Migrating toward the Equator with an 11 Year Period, ApJ, 575, L47-L50

Birch, A. C., Kosovichev, A. G., Price, G. H., Schlottmann, R. B., 2001, The Accuracy of the Born and Ray Approximations in Time-Distance Helioseismology, ApJ, 561, L229-L232

Birch, A. C., Kosovichev, A. G., Duvall, Jr., T. L., 2004, Sensitivity of Acoustic Wave Travel Times to Sound-Speed Perturbations in the Solar Interior, ApJ, 608, 580-600

Böning, V. G. A., 2017, Inferences of the deep solar meridional flow, Ph.D. thesis, Kiepenheuer-Institut für Sonnenphysik, Freiburg, Germany

Böning, V. G. A., Roth, M., Jackiewicz, J., Kholikov, S., 2017, Inversions for Deep Solar Meridional Flow Using Spherical Born Kernels, ApJ, 845, 2, 1707.08803

Braun, D. C., Birch, A. C., 2008, Prospects for the Detection of the Deep Solar Meridional Circulation, ApJ, 689, L161, 0810.0284

Braun, D. C., Fan, Y., 1998, Helioseismic Measurements of the Subsurface Meridional Flow, ApJ, 508, L105-L108

Braun, D. C., Duvall, T. L., J., Labonte, B. J., 1987, Acoustic Absorption by Sunspots, ApJ, 319, L27 
Broomhall, A. M., Chatterjee, P., Howe, R., Norton, A. A., Thompson, M. J., 2014, The Sun's Interior Structure and Dynamics, and the Solar Cycle, Space Sci. Rev., 186, 191$225,1411.5941$

Cameron, R. H., Schüssler, M., 2017, An update of Leighton's solar dynamo model, A\&A, 599, A52, 1611.09111

Cameron, R. H., Duvall, T. L., Schüssler, M., Schunker, H., 2018, Observing and modeling the poloidal and toroidal fields of the solar dynamo, A\&A, 609, A56, 1710.07126

Chabassier, J., Durufle, M., 2016, High Order Finite Element Method for solving Convected Helmholtz equation in radial and axisymmetric domains. Application to Helioseismology, Research Report RR-8893, Inria Bordeaux Sud-Ouest

Charbonneau, P., 2010, Dynamo Models of the Solar Cycle, Living Reviews in Solar Physics, 7, 3

Chen, R., Zhao, J., 2017, A Comprehensive Method to Measure Solar Meridional Circulation and the Center-to-limb Effect Using Time-Distance Helioseismology, ApJ, 849, $144,1709.07905$

Choudhuri, A. R., Schussler, M., Dikpati, M., 1995, The solar dynamo with meridional circulation., A\&A, 303, L29

Christensen-Dalsgaard, J., 2002, Helioseismology, Reviews of Modern Physics, 74, 1073-1129, astro-ph/0207403

Christensen-Dalsgaard, J., Duvall, T. L., J., Gough, D. O., Harvey, J. W., Rhodes, E. J., J., 1985, Speed of sound in the solar interior, Nature, 315, 378-382

Christensen-Dalsgaard, J., Gough, D. O., Thompson, M. J., 1991, The Depth of the Solar Convection Zone, ApJ, 378, 413

Christensen-Dalsgaard, J., Hansen, P. C., Thompson, M. J., 1993, Generalized Singular Value Decomposition Analysis of Helioseismic Inversions, MNRAS, 264, 541

Christensen-Dalsgaard, J., Dappen, W., Ajukov, S. V., Anderson, E. R., Antia, H. M., Basu, S., Baturin, V. A., Berthomieu, G., Chaboyer, B., Chitre, S. M., Cox, A. N., Demarque, P., Donatowicz, J., Dziembowski, W. A., Gabriel, M., Gough, D. O., Guenther, D. B., Guzik, J. A., Harvey, J. W., Hill, F., Houdek, G., Iglesias, C. A., Kosovichev, A. G., Leibacher, J. W., Morel, P., Proffitt, C. R., Provost, J., Reiter, J., Rhodes, Jr., E. J., Rogers, F. J., Roxburgh, I. W., Thompson, M. J., Ulrich, R. K., 1996, The Current State of Solar Modeling, Science, 272, 1286-1292

Couvidat, S., Schou, J., Hoeksema, J. T., Bogart, R. S., Bush, R. I., Duvall, T. L., Liu, Y., Norton, A. A., Scherrer, P. H., 2016, Observables Processing for the Helioseismic and Magnetic Imager Instrument on the Solar Dynamics Observatory, Sol. Phys., 291, 1887-1938, 1606.02368

Dahlen, F. A., Baig, A. M., 2002, Fréchet kernels for body-wave amplitudes, Geophys. J. Int., 150, 440-466 
Dalton, C. A., Ekström, G., 2006, Global models of surface wave attenuation, Journal of Geophysical Research (Solid Earth), 111, B05317

Dalton, C. A., Hjörleifsdóttir, V., Ekström, G., 2014, A comparison of approaches to the prediction of surface wave amplitude, Geophys. J. Int., 196, 386-404

Deubner, F. L., 1975, Observations of low wavenumber nonradial eigenmodes of the sun., A\&A, 44, 371-375

Duvall, T. L., J., 1979, Large-scale solar velocity fields., Sol. Phys., 63, 3-15

Duvall, T. L., J., Kosovichev, A. G., Scherrer, P. H., Bogart, R. S., Bush, R. I., de Forest, C., Hoeksema, J. T., Schou, J., Saba, J. L. R., Tarbell, T. D., 1997, Time-Distance Helioseismology with the MDI Instrument: Initial Results, Sol. Phys., 170, 63-73

Duvall, T. L., D’Silva, S., Jefferies, S. M., Harvey, J. W., Schou, J., 1996, Downflows under sunspots detected by helioseismic tomography, Nature, 379, 235-237

Duvall, Jr., T. L., 1995, Time-Distance Helioseismology: an Update, in GONG 1994. Helio- and Astro-Seismology from the Earth and Space CS-, (Eds.) R. K. Ulrich, E. J. Rhodes, Jr., W. Dappen, vol. 76, pp. Astron. Soc. Pacific, 465

Duvall, Jr., T. L., Jefferies, S. M., Harvey, J. W., Pomerantz, M. A., 1993, Time-distance helioseismology, Nature, 362, 430-432

Featherstone, N. A., Miesch, M. S., 2015, Meridional Circulation in Solar and Stellar Convection Zones, ApJ, 804, 67, 1501.06501

Fichtner, A., 2010, Full Seismic Waveform Modelling and Inversion, Springer Verlag

Fossat, E., Boumier, P., Corbard, T., Provost, J., Salabert, D., Schmider, F. X., Gabriel, A. H., Grec, G., Renaud, C., Robillot, J. M., Roca-Cortés, T., Turck-Chièze, S., Ulrich, R. K., Lazrek, M., 2017, Asymptotic g modes: Evidence for a rapid rotation of the solar core, A\&A, 604, A40, 1708.00259

Fournier, D., Gizon, L., Hohage, T., Birch, A. C., 2014, Generalization of the noise model for time-distance helioseismology, A\&A, 567, A137, 1406.5335

Fournier, D., Gizon, L., Holzke, M., Hohage, T., 2016, Pinsker estimators for local helioseismology: inversion of travel times for mass-conserving flows, Inverse Problems, 32, 105002, 1503.09084

Fournier, D., Hanson, C. S., Gizon, L., Barucq, H., 2018, Sensitivity kernels for timedistance helioseismology. Efficient computation for spherically symmetric solar models, A\&A, 616, A156, 1805.06141

Giles, P. M., 2000, Time-distance measurements of large-scale flows in the solar convection zone, Ph.D. thesis, STANFORD UNIVERSITY

Giles, P. M., Duvall, T. L., Scherrer, P. H., Bogart, R. S., 1997, A subsurface flow of material from the Sun's equator to its poles, Nature, 390, 52-54 
Gizon, L., 2004, Helioseismology of Time-Varying Flows Through The Solar Cycle, Sol. Phys., 224, 217-228

Gizon, L., 2006, Probing Convection and Solar Activity with Local Helioseismology, in SOHO-17. 10 Years of SOHO and Beyond SP-, vol. 617, pp. ESA, 5.1

Gizon, L., Birch, A. C., 2002, Time-Distance Helioseismology: The Forward Problem for Random Distributed Sources, ApJ, 571, 966-986

Gizon, L., Birch, A. C., 2004, Time-Distance Helioseismology: Noise Estimation, ApJ, $614,472-489$

Gizon, L., Birch, A. C., 2005, Local Helioseismology, Living Rev. Sol. Phys., 2, 6

Gizon, L., Rempel, M., 2008, Observation and Modeling of the Solar-Cycle Variation of the Meridional Flow, Sol. Phys., 251, 241-250,0803.0950

Gizon, L., Birch, A. C., Spruit, H. C., 2010, Local Helioseismology: Three-Dimensional Imaging of the Solar Interior, ARA\&A, 48, 289-338, 1001.0930

Gizon, L., Barucq, H., Duruflé, M., Hanson, C. S., Leguèbe, M., Birch, A. C., Chabassier, J., Fournier, D., Hohage, T., Papini, E., 2017, Computational helioseismology in the frequency domain: acoustic waves in axisymmetric solar models with flows, A\&A, 600, A35, 1611.01666

Gizon, L., Cameron, R. H., Pourabdian, M., Liang, Z.-C., Fournier, D., Birch, A. C., Hanson, C. S., 2020, Meridional flow in the Sun's convection zone is a single cell in each hemisphere, Science, 368, 1469-1472

Goldreich, P., Keeley, D. A., 1977, Solar seismology. II. The stochastic excitation of the solar p-modes by turbulent convection., ApJ, 212, 243-251

Haber, D. A., Hindman, B. W., Toomre, J., Bogart, R. S., Larsen, R. M., Hill, F., 2002, Evolving Submerged Meridional Circulation Cells within the Upper Convection Zone Revealed by Ring-Diagram Analysis, ApJ, 570, 855-864

Hanasoge, S. M., Tromp, J., 2014, Full Waveform Inversion for Time-Distance Helioseismology, ApJ, 784, 69, 1401.7603

Hansen, P., 2010, Discrete Inverse Problems: Insight and Algorithms, Society for Industrial and Applied Mathematics SIAM, ISBN 978-0-89871-696-2

Hartlep, T., Zhao, J., Kosovichev, A. G., Mansour, N. N., 2013, Solar Wave-field Simulation for Testing Prospects of Helioseismic Measurements of Deep Meridional Flows, ApJ, 762, 132, 1209.4602

Harvey, J., Tucker, R., Britanik, L., 1998, High Resolution Upgrade of the GONG Instruments, in Structure and Dynamics of the Interior of the Sun and Sun-like Stars, (Ed.) S. Korzennik, vol. 418 of ESA Special Publication, p. 209 
Harvey, J. W., Hill, F., Hubbard, R. P., Kennedy, J. R., Leibacher, J. W., Pintar, J. A., Gilman, P. A., Noyes, R. W., Title, A. M., Toomre, J., Ulrich, R. K., Bhatnagar, A., Kennewell, J. A., Marquette, W., Patron, J., Saa, O., Yasukawa, E., 1996, The Global Oscillation Network Group (GONG) Project, Science, 272, 1284-1286

Hathaway, D. H., 1996, Doppler Measurements of the Sun's Meridional Flow, ApJ, 460, 1027

Hathaway, D. H., Rightmire, L., 2010, Variations in the Sun's Meridional Flow over a Solar Cycle, Science, 327, 1350

Hathaway, D. H., Upton, L., 2014, The solar meridional circulation and sunspot cycle variability, Journal of Geophysical Research (Space Physics), 119, 3316-3324, 1404. 5893

Hill, F., 1988, Rings and Trumpets-Three-dimensional Power Spectra of Solar Oscillations, ApJ, 333, 996

Hotta, H., 2017, Solar Overshoot Region and Small-scale Dynamo with Realistic Energy Flux, ApJ, 843, 52, 1706.06413

Houdek, G., 2006, Stochastic excitation and damping of solar-like oscillations, in Proceedings of SOHO 18/GONG 2006/HELAS I, Beyond the spherical Sun, vol. 624 of ESA Special Publication, p. 28

Houdek, G., Dupret, M.-A., 2015, Interaction Between Convection and Pulsation, Living Reviews in Solar Physics, 12, 8, 1601.03913

Howe, R., 2009, Solar Interior Rotation and its Variation, Living Reviews in Solar Physics, 6, 1, 0902.2406

Hughes, A. L. H., Jain, K., Kholikov, S., the NISP Solar Interior Group, 2016, GONG ClassicMerge: Pipeline and Product, arXiv e-prints, arXiv:1603.00836, 1603.00836

Hughes, S. J., Pijpers, F. P., Thompson, M. J., 2007, Optimized data masks for focussed solar tomography: background and artificial diagnostic experiments, A\&A, 468, 341351

Jackiewicz, J., Birch, A. C., Gizon, L., Hanasoge, S. M., Hohage, T., Ruffio, J.-B., Švanda, M., 2012, Multichannel Three-Dimensional SOLA Inversion for Local Helioseismology, Sol. Phys., 276, 19-33, 1109.2712

Jackiewicz, J., Serebryanskiy, A., Kholikov, S., 2015, Meridional Flow in the Solar Convection Zone. II. Helioseismic Inversions of GONG Data, ApJ, 805, 133, 1504 . 08071

Jensen, J. M., 2001, Helioseismic Time-Distance Inversion, Ph.D. thesis, University of Aarhus, Denmark

Kholikov, S., Hill, F., 2014, Meridional-Flow Measurements from Global Oscillation Network Group Data, Sol. Phys., 289, 1077-1084 
Kitchatinov, L. L., 2016, Meridional circulation in the sun and stars, Geomagnetism and Aeronomy, 56, 945-951, 1603.07852

Korda, D., Švanda, M., Zhao, J., 2019, Comparison of time-distance inversion methods applied to SDO/HMI Dopplergrams, A\&A, 629, A55, 1908.03950

Kosovichev, A. G., 1996, Tomographic Imaging of the Sun's Interior, ApJ, 461, L55

Kosovichev, A. G., Duvall, Jr., T. L., 1997, Acoustic tomography of solar convective flows and structures, in SCORe'96 : Solar Convection and Oscillations and their Relationship, (Eds.) F. P. Pijpers, J. Christensen-Dalsgaard, C. S. Rosenthal, vol. 225 of Astrophysics and Space Science Library, pp. 241-260, 1806.03273

Kosovichev, A. G., Schou, J., Scherrer, P. H., Bogart, R. S., Bush, R. I., Hoeksema, J. T., Aloise, J., Bacon, L., Burnette, A., de Forest, C., Giles, P. M., Leibrand, K., Nigam, R., Rubin, M., Scott, K., Williams, S. D., Basu, S., Christensen-Dalsgaard, J., Dappen, W., Rhodes, Jr., E. J., Duvall, Jr., T. L., Howe, R., Thompson, M. J., Gough, D. O., Sekii, T., Toomre, J., Tarbell, T. D., Title, A. M., Mathur, D., Morrison, M., Saba, J. L. R., Wolfson, C. J., Zayer, I., Milford, P. N., 1997, Structure and Rotation of the Solar Interior: Initial Results from the MDI Medium-L Program, Sol. Phys., 170, 43-61

Langfellner, J., Gizon, L., Birch, A. C., 2015, Spatially resolved vertical vorticity in solar supergranulation using helioseismology and local correlation tracking, A\&A, 581, A67, 1504.00223

Leibacher, J. W., Stein, R. F., 1971, A New Description of the Solar Five-Minute Oscillation, Astrophys. Lett., 7, 191-192

Leighton, R. B., Noyes, R. W., Simon, G. W., 1962, Velocity Fields in the Solar Atmosphere. I. Preliminary Report., ApJ, 135, 474

Liang, Z.-C., Chou, D.-Y., 2015, Effects of Solar Surface Magnetic Fields on the TimeDistance Analysis of Solar Subsurface Meridional Flows, ApJ, 805, 165

Liang, Z.-C., Gizon, L., Schunker, H., Philippe, T., 2013, Helioseismology of sunspots: defocusing, folding, and healing of wavefronts, A\&A, 558, A129

Liang, Z.-C., Birch, A. C., Duvall, Jr., T. L., Gizon, L., Schou, J., 2017, Comparison of acoustic travel-time measurements of solar meridional circulation from SDO/HMI and SOHO/MDI, A\&A, 601, A46, 1704.00475

Liang, Z.-C., Gizon, L., Birch, A. C., Duvall, T. L., Rajaguru, S. P., 2018, Solar meridional circulation from twenty-one years of SOHO/MDI and SDO/HMI observations. Helioseismic travel times and forward modeling in the ray approximation, A\&A, 619, A99, 1808.08874

Liang, Z.-C., Gizon, L., Birch, A. C., Duvall, T. L., 2019, Time-distance helioseismology of solar Rossby waves, A\&A, 626, A3, 1812.07413

Lin, C.-H., Chou, D.-Y., 2018, Solar-cycle Variations of Meridional Flows in the Solar Convection Zone Using Helioseismic Methods, ApJ, 860, 48 
Lindsey, C., Braun, D. C., 1990, Helioseismic Imaging of Sunspots at Their Antipodes, Sol. Phys., 126, 101-115

Mandal, K., Hanasoge, S. M., Rajaguru, S. P., Antia, H. M., 2018, Helioseismic Inversion to Infer the Depth Profile of Solar Meridional Flow Using Spherical Born Kernels, ApJ, $863,39,1807.00314$

Miesch, M. S., 2005, Large-Scale Dynamics of the Convection Zone and Tachocline, Living Reviews in Solar Physics, 2, 1

Miesch, M. S., Featherstone, N. A., Rempel, M., Trampedach, R., 2012, On the Amplitude of Convective Velocities in the Deep Solar Interior, ApJ, 757, 128, 1205.1530

Moradi, H., Hanasoge, S. M., 2010, Deep-Focus Diagnostics of Sunspot Structure, Astrophysics and Space Science Proceedings, 19, 378-385, 1101.0125

Nagashima, K., Fournier, D., Birch, A. C., Gizon, L., 2017, The amplitude of the crosscovariance function of solar oscillations as a diagnostic tool for wave attenuation and geometrical spreading, A\&A, 599, A111, 1612.08991

Nolet, G., Dahlen, F. A., Montelli, R., 2005, Traveltimes and amplitudes of seismic waves: A re-assessment, Washington DC American Geophysical Union Geophysical Monograph Series, 157, 37-47

Patron, J., Hill, F., Rhodes, E. J., J., Korzennik, S. G., Cacciani, A., 1995, Velocity Fields within the Solar Convection Zone: Evidence from Oscillation Ring Diagram Analysis of Mount Wilson Dopplergrams, ApJ, 455, 746

Pijpers, F. P., Thompson, M. J., 1994, The SOLA method for helioseismic inversion, A\&A, 281, 231-240

Pipin, V. V., Kosovichev, A. G., 2018, On the Origin of the Double-cell Meridional Circulation in the Solar Convection Zone, ApJ, 854, 67, 1708.03073

Rajaguru, S. P., Antia, H. M., 2015, Meridional Circulation in the Solar Convection Zone: Time-Distance Helioseismic Inferences from Four Years of HMI/SDO Observations, ApJ, 813, 114, 1510.01843

Ruediger, G., 1989, Differential rotation and stellar convection. Sun and the solar stars

Scherrer, P. H., Schou, J., Bush, R. I., Kosovichev, A. G., Bogart, R. S., Hoeksema, J. T., Liu, Y., Duvall, T. L., Zhao, J., Title, A. M., Schrijver, C. J., Tarbell, T. D., Tomczyk, S., 2012, The Helioseismic and Magnetic Imager (HMI) Investigation for the Solar Dynamics Observatory (SDO), Sol. Phys., 275, 207-227

Schou, J., Antia, H. M., Basu, S., Bogart, R. S., Bush, R. I., Chitre, S. M., ChristensenDalsgaard, J., Di Mauro, M. P., Dziembowski, W. A., Eff-Darwich, A., Gough, D. O., Haber, D. A., Hoeksema, J. T., Howe, R., Korzennik, S. G., Kosovichev, A. G., Larsen, R. M., Pijpers, F. P., Scherrer, P. H., Sekii, T., Tarbell, T. D., Title, A. M., Thompson, M. J., Toomre, J., 1998, Helioseismic Studies of Differential Rotation in the Solar 
Envelope by the Solar Oscillations Investigation Using the Michelson Doppler Imager, ApJ, 505, 390-417

Schou, J., Scherrer, P. H., Bush, R. I., Wachter, R., Couvidat, S., Rabello-Soares, M. C., Bogart, R. S., Hoeksema, J. T., Liu, Y., Duvall, T. L., Akin, D. J., Allard, B. A., Miles, J. W., Rairden, R., Shine, R. A., Tarbell, T. D., Title, A. M., Wolfson, C. J., Elmore, D. F., Norton, A. A., Tomczyk, S., 2012, Design and Ground Calibration of the Helioseismic and Magnetic Imager (HMI) Instrument on the Solar Dynamics Observatory (SDO), Sol. Phys., 275, 229-259

Schunker, H., Schou, J., Gaulme, P., Gizon, L., 2018, Fragile Detection of Solar g-Modes by Fossat et al., Sol. Phys., 293, 95, 1804.04407

Spruit, H. C., 2003, Origin of the torsional oscillation pattern of solar rotation, Sol. Phys., 213, 1-21, astro-ph/0209146

Tarantola, A., 2005, Inverse Problem Theory and Methods for Model Parameter Estimation, Other titles in applied mathematics, Society for Industrial and Applied Mathematics, ISBN 9780898715729

Thompson, M. J., Toomre, J., Anderson, E. R., Antia, H. M., Berthomieu, G., Burtonclay, D., Chitre, S. M., Christensen-Dalsgaard, J., Corbard, T., De Rosa, M., Genovese, C. R., Gough, D. O., Haber, D. A., Harvey, J. W., Hill, F., Howe, R., Korzennik, S. G., Kosovichev, A. G., Leibacher, J. W., Pijpers, F. P., Provost, J., Rhodes, E. J., J., Schou, J., Sekii, T., Stark, P. B., Wilson, P. R., 1996, Differential Rotation and Dynamics of the Solar Interior, Science, 272, 1300-1305

Thompson, M. J., Christensen-Dalsgaard, J., Miesch, M. S., Toomre, J., 2003, The Internal Rotation of the Sun, ARA\&A, 41, 599-643

Tong, J., Dahlen, F. A., Nolet, G., Marquering, H., 1998, Diffraction effects upon finitefrequency travel times: A simple 2-D example, Geophys. Res. Lett., 25, 1983-1986

Ulrich, R. K., 1970, The Five-Minute Oscillations on the Solar Surface, ApJ, 162, 993

Ulrich, R. K., 2010, Solar Meridional Circulation from Doppler Shifts of the Fe I Line at $5250 \AA$ as Measured by the 150 -foot Solar Tower Telescope at the Mt. Wilson Observatory, ApJ, 725, 658-669, 1010.0487

Virieux, J., Operto, S., 2009, An overview of full-waveform inversion in exploration geophysics, GEOPHYSICS, 74, WCC1-WCC26, https://doi.org/10.1190/1. 3238367

Vorontsov, S. V., Christensen-Dalsgaard, J., Schou, J., Strakhov, V. N., Thompson, M. J., 2002, Helioseismic Measurement of Solar Torsional Oscillations, Science, 296, 101103

Švanda, M., Gizon, L., Hanasoge, S. M., Ustyugov, S. D., 2011, Validated helioseismic inversions for 3D vector flows, A\&A, 530, A148, 1104.4083 
Wang, Y. M., Sheeley, N. R., J., Nash, A. G., 1991, A New Solar Cycle Model Including Meridional Circulation, ApJ, 383, 431

Woodard, M. F., 2009, Helioseismic Measurement of Large-Scale Solar Flows, in SolarStellar Dynamos as Revealed by Helio- and Asteroseismology: GONG 2008/SOHO 21, (Eds.) M. Dikpati, T. Arentoft, I. González Hernández, C. Lindsey, F. Hill, vol. 416 of Astronomical Society of the Pacific Conference Series, p. 15

Zhao, J., Kosovichev, A. G., 2004, Torsional Oscillation, Meridional Flows, and Vorticity Inferred in the Upper Convection Zone of the Sun by Time-Distance Helioseismology, ApJ, 603, 776-784

Zhao, J., Nagashima, K., Bogart, R. S., Kosovichev, A. G., Duvall, T. L., J., 2012, Systematic Center-to-limb Variation in Measured Helioseismic Travel Times and its Effect on Inferences of Solar Interior Meridional Flows, ApJ, 749, L5, 1203.1904

Zhao, J., Bogart, R. S., Kosovichev, A. G., Duvall, Jr., T. L., Hartlep, T., 2013, Detection of Equatorward Meridional Flow and Evidence of Double-cell Meridional Circulation inside the Sun, ApJ, 774, L29, 1307.8422

Zhao, J., Kosovichev, A. G., Bogart, R. S., 2014, Solar Meridional Flow in the Shallow Interior during the Rising Phase of Cycle 24, ApJ, 789, L7, 1406.2735 



\section{Scientific contributions}

\section{Refereed publications}

- M. Pourabdian, D. Fournier, and L. Gizon: Comparison of travel-time and amplitude measurements for deep-focusing time-distance helioseismology, Solar Physics, 293, 66, 2018.

- L. Gizon, R. H. Cameron, M. Pourabdian, Z.-C. Liang, D. Fournier, A. C. Birch, and C. S. Hanson: Meridional flow in the Sun's convection zone is a single cell in each hemisphere, Science, 368 (6498), 1469-1472, 2020.

\section{Conference contributions}

- 14th International Conference on Mathematical and Numerical Aspects of Wave Propagation (WAVES2019), Vienna, Austria, August 25-30, 2019

Talk: Comparison of full-waveform and travel-time inversions in helioseismology.

- Second Max Planck Partner Group Workshop on Solar Physics, Mumbai, India, March 17-20, 2019

Talk: Inversions of two-point correlations.

- XXXth General Assembly of the International Astronomical Union, Vienna, Austria, August 20-31, 2018

Poster: Optimal averaging for helioseismic measurements using the singular value decomposition.

- Annual Meeting of the German Astronomical Society (Astronomische Gesellschaft), Göttingen, Germany, September 18-22, 2017

Poster: Comparison between travel-time and amplitude measurements using deepfocusing time-distance helioseismology.

- Rocks \& Stars II conference, Göttingen, Germany, September 13-16, 2017

Talk: Deep focusing in time-distance helioseismology. 



\section{Acknowledgements}

First and foremost, I would like to thank my supervisor Laurent Gizon without whom this dissertation would not have been possible to be delivered successfully. I am really grateful for his advice, guidance, help, and support during my Ph.D. journey. I also thank Thorsten Hohage to be in my TAC and guide me to find my way towards inverse problems. A special thanks goes out to Damien Fournier who was not only a member of my TAC, but rather who has continuously and tirelessly helped me through my Ph.D. and answered my questions.

I would also like to thank Laurent Gizon, Thorsten Hohage, Stefan Dreizler, Andreas Tilgner, Wolfram Kollatschny, and Ramin Yahyapour for being in the thesis defense committee.

I also thank the International Max Planck Research School (IMPRS) for Solar System Science at the University of Göttingen and the Max Planck Institute for Solar System Research (MPS) for funding my doctorate studies and giving me the opportunity to accomplish this work. In particular, I gratefully thank Sonja Schuh as the IMRPS coordinator who helped and guided me to fulfill all the regulations and requirements of my Ph.D. studies.

I would also like to thank all the people in the Solar and Stellar Interiors Department at the MPS. Big thank you to Zhi-Chao Liang for the data analysis and Chris S. Hanson for the kernels. I also thank Thomas L. Duvall, Robert H. Cameron, Aaron C. Birch, Jesper Schou, and Vincent G. A. Böning for their constructive and helpful discussions for my research. Thanks to all my friends including my fellow Ph.D. students at the MPS who are magnificent and always nice and supportive in all ups and downs.

The MDI data used in this thesis are from SOHO which is a project of international cooperation between ESA and NASA. This thesis also used GONG data obtained by the NSO Integrated Synoptic Program (NISP), managed by the National Solar Observatory, the Association of Universities for Research in Astronomy (AURA), Inc. under a cooperative agreement with the National Science Foundation. The HMI data are courtesy of NASA/SDO and the HMI science team.

Last but not least, I would like to thank my beloved family, my great parents, Sima and Ali, and my lovely brother, Moein. I gratefully appreciate your love, endless support and everything you have done for me throughout my life. 



\section{Curriculum vitae}

\section{Personal}

Name: $\quad$ Majid Pourabdian

Date of birth: $\quad$ February 01, 1990

Place of birth: Rasht, Iran

Nationality: Iranian

ORCID iD: https://orcid.org/0000-0002-5368-7796

\section{Education}

2015 - 2020: $\quad$ Ph.D. in Physics

International Max Planck Research School for Solar System Science at the University of Göttingen, Göttingen, Germany

Thesis: Inverse problems in local helioseismology

Supervisors: Prof. Dr. Laurent Gizon, Prof. Dr. Thorsten Hohage, and Dr. Damien Fournier

2012 - 2015: $\quad$ M.Sc. in Aerospace Engineering

Department of Aerospace Engineering, Sharif University of Technology, Tehran, Iran

Thesis: Numerical modeling of liquid jet breakup using smoothed particle hydrodynamics $(\mathrm{SPH})$

Supervisors: Prof. Dr. Mohammad Reza Morad and Prof. Dr. Pourya Omidvar

2008 - 2012: $\quad$ B.Sc. in Aerospace Engineering

Aerospace Engineering Department, Amirkabir University of Technology (Tehran Polytechnic), Tehran, Iran

Thesis: Aerodynamic simulation of flow over an aircraft

Supervisor: Prof. Dr. Mahmoud Mani 